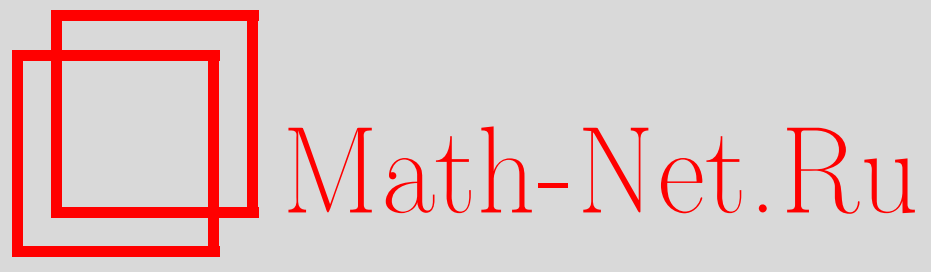

А. И. Буфетов, Предельные теоремы для специальных потоков над преобразованиями Вершика, УМH, 2013, том 68, выпуск 5, 3-80

DOI: https://doi.org/10.4213/rm9557

Использование Общероссийского математического портала Math-Net.Ru подразумевает, что вы прочитали и согласны с пользовательским соглашением http://www . mathnet.ru/rus/agreement

Параметры загрузки:

IP : 52.6 .47 .48

26 апреля 2023 г., 09:12:14

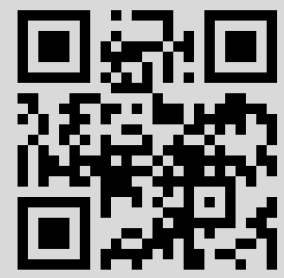




\title{
Предельные теоремы для специальных потоков над преобразованиями Вершика
}

\begin{abstract}
А. И. Буфетов
В работе получены асимптотическое разложение эргодического интеграла и предельная теорема для специальных потоков над преобразованиями Вершика.

Библиография: 49 названий.

Ключевые слова: преобразования Вершика, ренормализация, скорость сходимости в эргодической теореме, предельные теоремы, конечно аддитивные инвариантные меры, поток Тейхмюллера, инвариантные обобщенные функции Форни, коцикл Концевича-Зорича.
\end{abstract}

DOI: $10.4213 / \mathrm{rm} 9557$

\section{СОДЕРЖАНИЕ}

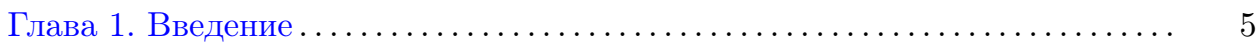

1.1. Марковские компакты . . . . . . . . . . . . . . . . . . . . . . . . . . . . . . 7

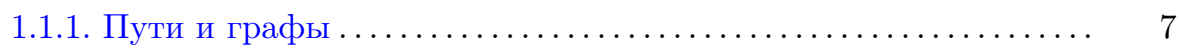

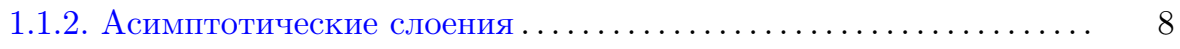

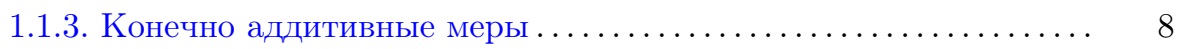

1.1.4. Меры прямого произведения и двойственность ............ 10

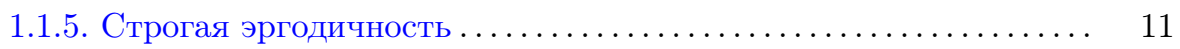

1.1.6. Слабо липшицевы функции ......................... 12

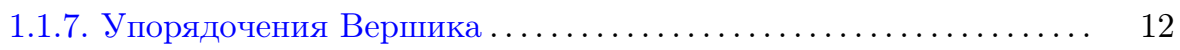

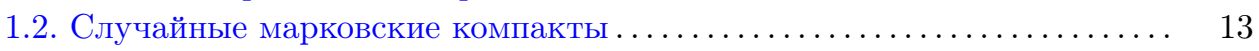

1.2.1. Пространство марковских компактов .................. 13

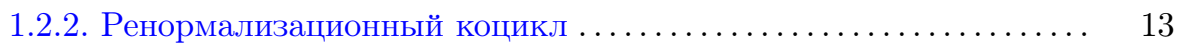

1.2.3. Приближение слабо липшицевых функций ................ 14

1.2.4. Транспонированный коцикл и двойственность ............ 14

Автор поддержан проектом A*MIDEX (№ ANR-11-IDEX-0001-02), профинансированным программой "Investissements d'Avenir" Правительства Французской Республики и подотчетным Французскому Национальному Агентству Исследований (ANR). Автор - стипендиат Фонда Альфреда Слоуна. Работа над этой статьей поддерживалась программой "Динамические системы и теория управления" Президиума Российской академии наук, грантами Президента РФ МК-4893.2010.1 и МK-6734.2012.1, грантами РФФИ 11-01-00654, 12-01-33020, 12-01-31284, грантом РФФИ-CNRS 10-01-93115-НЦНИЛ, Национальным научным фондом (грант DMS 0604386), Фондом Эдгара Оделла Ловетта в Университете Райса. 
Глава 2. Символическая аппроксимационная теорема.............. 16

2.1. Подготовительная аппроксимационная лемма................ 16

2.1.1. Случай одностороннего марковского компакта ............ 16

2.1.2. Двойственность .................................. 18

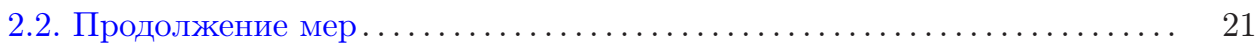

2.2.1. Кольцо хорошо приближаемых дуг .................. 21

2.2.2. Продолжение на дуги при наличии упорядочения Вершика... 22

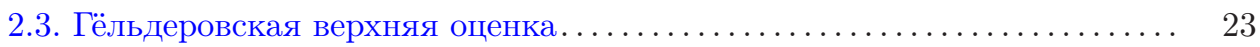

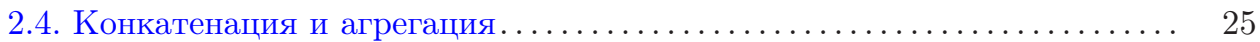

2.5. Символические потоки . . . . . . . . . . . . . . . . . . . . . . . 26

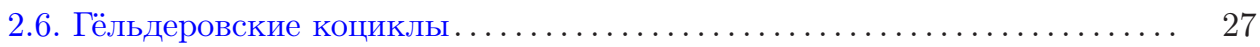

2.7. Сбалансированные, регулярные по Ляпунову и гиперболические мар-

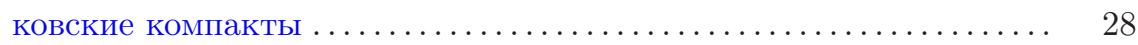

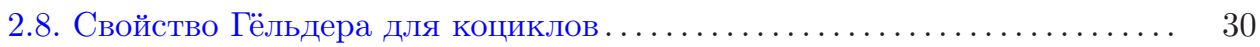

2.8.1. Верхняя оценка............................... 30

2.8.2. Логарифмическая асимптотика на бесконечности.......... 30

2.8.3. Математическое ожидание и дисперсия для гёльдеровских ко-

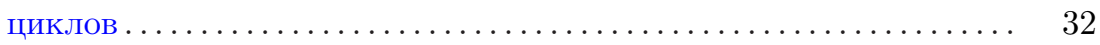

2.9. Приближение слабо липшицевых функций .................. 32

2.10. Аппроксимационная теорема для случайных марковских компактов 34

2.10.1. Косые произведения, сопоставленные сдвигу и ренормализа-

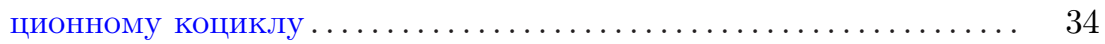

2.10.2. Свойства случайных марковских компактов ............ 35

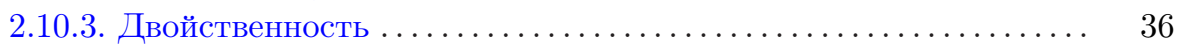

2.10.4. Сбалансированные случайные марковские компакты и аппроксимационная теорема .......................... 36

2.10.5. Гиперболические случайные марковские компакты . . . . . . . 37

Глава 3. Ренормализационный поток на пространстве марковских компак-

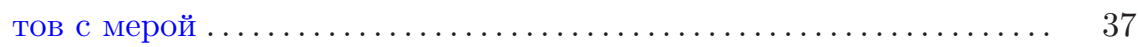

3.1. Пространство марковских компактов с мерой................ 37

3.1.1. Ренормализационный поток и ренормализационный коцикл.. 38

3.1.2. Характеризация конечно аддитивных мер ............... 39

3.1.3. Пространство "символических потоков переноса" ........... 42

3.2. Предельные теоремы в случае простого второго показателя Ляпунова 43

3.2.1. Главный член в асимптотике эргодического интеграла ....... 43

3.2.2. Рост дисперсии .................................. 44

3.2.3. Формулировка и доказательство предельной теоремы ........ 46

3.2.4. Омега-предельные множества предельных распределений... 47

3.3. Предельные теоремы в общем случае .................... 49

3.3.1. Формулировка и доказательство предельной теоремы ........ 49

3.3.2. Атомы предельных распределений ................... 51

3.3.3. Накопление к нулю для предельных распределений ......... 53

3.4. Эргодические средние для автоморфизмов Вершика............. 55

3.4.1. Пространство односторонних марковских компактов ....... 55

3.4.2. Автоморфизм Вершика ....................... 57 
Глава 4. Марковские компакты и абелевы дифференциалы . . . . . . . . . . 57

4.1. Символическое кодирование для потоков на плоских поверхностях . . 57

4.1.1. Перекладывания отрезков как автоморфизмы Вершика...... 57

4.1.2. Потоки на плоских поверхностях как символические потоки .. 60

4.2. Пространство зашнурованных прямоугольников Вича........... 62

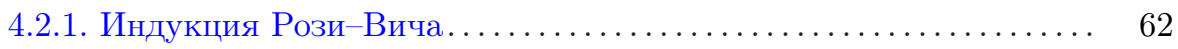

4.2.2. Построение зашнурованных прямоугольников ............ 64

4.2.3. Зашнурованные прямоугольники и абелевы дифференциалы. . 64

4.2.4. Пространство зашнурованных прямоугольников .......... 64

4.2.5. Соответствие между коциклами .................. 66

4.3. Зашнурованные прямоугольники и марковские компакты ......... 69

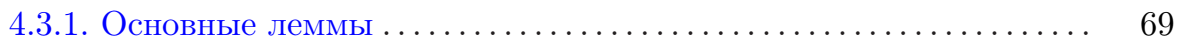

4.3.2. Коды Рози-Вича зашнурованных прямоугольников ......... 71

4.3.3. Марковский компакт, соответствующий зашнурованному пря-

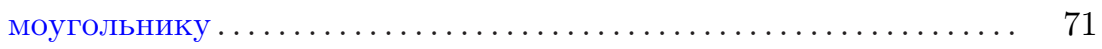

4.3.4. Свойства символического кодирования ................. 72

Приложение А. О мультипликативной эргодической теореме Оселедца... 75

А.1. Теорема Оселедца-Песина о редукции .................. 75

А.2. Лемма Вианы о простоте старшего показателя Ляпунова...... 76

Приложение В. Соответствие между символическими и геометрическими

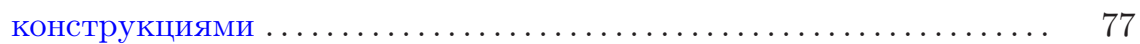

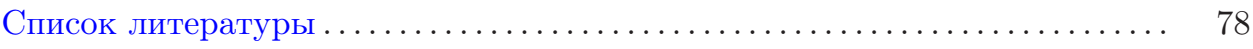

\section{Глава 1. Введение}

Целью настоящей работы является получение асимптотического разложения для эргодического интеграла и предельной теоремы для специальных потоков над автоморфизмами Вершика. Ключевую роль в доказательстве играет построение специального семейства конечно аддитивных мер на асимптотических слоениях марковского компакта.

Произвольному эргодическому автоморфизму пространства Лебега А. М. Вершик сопоставил в [45] последовательность башен Рохлина, пересекающихся марковским образом и таких, что соответствующая последовательность измеримых разбиений сходится к разбиению на точки. Другими словами, исходный эргодический автоморфизм представляется как автоморфизм Вериика одностороннего марковского компакта.

Кодирование Вершика строится в явном виде для подстановочных динамических систем (теорема Вершика-Лившица [46]) и для перекладываний отрезков (в этом случае требуемая последовательность рохлинских башен получается из индукционного алгоритма Рози-Вича).

Из самого определения автоморфизма Вершика видно, что орбитальное отношение эквивалентности для него совпадает с хвостовым отношением эквивалентности марковского компакта. В данной работе представлена конструкция 
сохраняющих меру потоков, орбиты которых являются листами асимптотических слоений сохраняющих меру потоков на двустороннем марковском компакте. В частности, эта конструкция дает символическое кодирование для минимальных потоков переноса на плоских поверхностях. Целью настоящей работы является изучение скорости сходимости эргодических интегралов для таких символических потоков.

Двустороннему марковскому компакту соответствует пространство конечно аддитивных мер, определенных на листах асимптотических слоений и инвариантных относительно голономии вдоль другого асимптотического слоения. Мы покажем, что при некоторых дополнительных предположениях о графах, задающих марковский компакт, эти меры управляют асимптотическим поведением эргодического интеграла. Отметим также, что пространства конечно аддитивных мер на листах двух асимптотических слоений двойственны друг другу.

Формализм, представленный в данной работе, применим к случаю периодического марковского компакта, который можно рассматривать как пространство ориентированных путей в некотором конечном графе. К существенно более общему классу марковских компактов, асимптотическое поведение которых тем не менее сходно с поведением в периодическом случае, приводит рассмотрение последовательностей графов, задающих компакт и получающихся как реализации эргодического стационарного процесса.

Более точно, рассмотрим пространство марковских компактов, т. е. пространство бесконечных в обе стороны последовательностей ориентированных графов с фиксированным числом вершин. Число их ребер, однако, не фиксируется, оно может быть даже неограниченным.

На пространстве марковских компактов заданы естественная динамическая система, сдвиг, и естественный ренормализационный коцикл над сдвигом, задаваемый матрицами смежности графов. Снабдим пространство марковских компактов эргодической вероятностной мерой, инвариантной относительно сдвига; наше основное предположение будет состоять в том, что теорема Ocеледца применима к ренормализационному коциклу по отношению к нашей мере. В этом случае пространство конечно аддитивных мер, удовлетворяющих естественному дополнительному условию гёльдеровости, естественным образом отождествляется с неустойчивым оселедцевским подпространством ренормализационного коцикла, и эргодические интегралы для наших символических потоков допускают асимптотическое разложение в терминах этих конечно аддитивных мер.

Из полученного асимптотического разложения также вытекает предельная теорема для этих потоков. При дополнительных предположениях на стационарную меру на пространстве марковских компактов эргодические интегралы липшицевых функций, нормализованные так, чтобы они имели единичную дисперсию, не сходятся по распределению. Вместо этого для почти всех марковских компактов в нашем пространстве имеется "асимптотическое разложение" (свое для каждого из них), и распределение эргодического интеграла, рассматриваемого как функция времени, сходится к траектории "асимптотического 
распределения" относительно сдвига. Эта сходимость является экспоненциально быстрой в метриках Леви-Прохорова и Канторовича-Рубинштейна.

Основной пример, мотивировавший данную работу, - это потоки переноса на типичных плоских поверхностях. Теорема 1 в [14] дает асимптотическое разложение эргодических интегралов, а теорема 2 - предельную теорему для потоков переноса. Доказательства в [14] используют формализм пространства зашнурованных прямоугольников Вича. В конце настоящей работы мы получим теоремы 1 и 2 из [14] как следствия их более общих символических аналогов. Результаты работы анонсированы без доказательств в [12], [13]. В приложении В указаны соответствия между основными символическими и геометрическими конструкциями.

Я глубоко благодарен А. Авиле, К. Брессо, А. М. Вершику, У. О. Вичу, И. В. Вьюгину, Б. М. Гуревичу, А.В. Зоричу, Ж.-К. Йоккозу, А.Б. Катку, А. В. Клименко, С. Б. Куксину, К. Макмюллену, В. И. Оселедцу, Я. Г. Синаю, Дж. Форни за полезные обсуждения. Я глубоко благодарен Ю. С. Ильяшенко, Х. Крюгеру, Э. Ланно, С. Мкртчяну, Р. Райему, Й. Чайке, П. Юберу за многочисленные предложения, позволившие улучшить изложение. Я глубоко благодарен Х. Накаде, указавшему мне ссылку на работу С. Ито [26], и Б. Соломяку, указавшему ссылки на работу Ж.-М. Дюмона, Т. Камае, С. Такахаси [16] и работу Т. Камае [27]. Я глубоко благодарен Н. Козину, Д. Онгу, Р. Ульмаскулову и С. Шарахову, которые набрали части этой рукописи. Часть настоящей работы была написана во время моего визита в Математический институт им. Макса Планка в Бонне.

\section{1. Марковские компакты}

1.1.1. Пути и графы. Зафиксируем число $m \in \mathbb{N}$. Пусть $Г$ - ориентированный граф с $m$ вершинами $\{1, \ldots, m\}$, возможно с кратными ребрами. Будем предполагать, что из каждой вершины выходит хотя бы одно ребро и что в каждую вершину входит хотя бы одно ребро. Множество всех ориентированных графов на $m$ вершинах, удовлетворяющих этому условию, обозначим $\mathfrak{G}$.

Пусть $\mathscr{E}(\Gamma)$ - множество ребер графа Г. Для ребра $e \in \mathscr{E}(\Gamma)$ будем обозначать $I(e)$ его начальную вершину, а $F(e)$ - его конечную вершину. Пусть $A(\Gamma)-$ матрица инцидентности графа $\Gamma$, задаваемая формулой

$$
A_{i j}(\Gamma)=\#\{e \in \mathscr{E}(\Gamma): I(e)=i, F(e)=j\}
$$

Пусть задана последовательность $\Gamma_{n}, n \in \mathbb{Z}$, графов из множества $\mathfrak{G}$. Этой последовательности сопоставим марковский компакт (ср. с [1], [2], [18], [44], [45]) путей в нашей последовательности графов:

$$
X=\left\{x=\ldots x_{-n} \ldots x_{n} \ldots, x_{n} \in \mathscr{E}\left(\Gamma_{n}\right), F\left(x_{n+1}\right)=I\left(x_{n}\right)\right\} .
$$

Будем полагать $A_{n}(X)=A\left(\Gamma_{n}\right)$. 
1.1.2. Асимптотические слоения. Для $x \in X, n \in \mathbb{Z}$ определим множества

$$
\begin{array}{ll}
\gamma_{n}^{+}(x)=\left\{x^{\prime} \in X: x_{t}^{\prime}=x_{t}, t \geqslant n\right\}, & \gamma_{n}^{-}(x)=\left\{x^{\prime} \in X: x_{t}^{\prime}=x_{t}, t \leqslant n\right\}, \\
\gamma_{\infty}^{+}(x)=\bigcup_{n \in \mathbb{Z}} \gamma_{n}^{+}(x), & \gamma_{\infty}^{-}(x)=\bigcup_{n \in \mathbb{Z}} \gamma_{n}^{-}(x) .
\end{array}
$$

Множества $\gamma_{\infty}^{+}(x)$ - это листы асимптотического слоения $\mathscr{F}+(X)$ на $X$, соответствующего бесконечно удаленному будущему, а множества $\gamma_{\infty}^{-}(x)$ - листы асимптотического слоения $\mathscr{F}^{-}(X)$ на $X$, соответствующего бесконечно удаленному прошлому.

Обозначим $\mathfrak{C}_{n}^{+}(X)$, где $n \in \mathbb{Z}$, семейство всех подмножеств $X$ вида $\gamma_{n}^{+}(x)$, $x \in X$; аналогично, пусть $\mathfrak{C}_{n}^{-}(X)$ - семейство всех подмножеств вида $\gamma_{n}^{-}(x)$.

По определению, семейства $\mathfrak{C}_{n}^{+}(X), \mathfrak{C}_{n}^{-}(X)$ являются полукольцами. Определим теперь семейства

$$
\mathfrak{C}^{+}(X)=\bigcup_{n \in \mathbb{Z}} \mathfrak{C}_{n}^{+}(X), \quad \mathfrak{C}^{-}(X)=\bigcup_{n \in \mathbb{Z}} \mathfrak{C}_{n}^{-}(X) .
$$

Поскольку каждый элемент $\mathfrak{C}_{n}^{+}$является дизъюнктным объединением элементов $\mathfrak{C}_{n+1}^{+}$, семейство $\mathfrak{C}^{+}$также является полукольцом. То же самое, конечно, справедливо и для $\mathfrak{C}^{-}$.

Цилиндрами в $X$ называются подмножества вида

$$
\left\{x: x_{n+1}=e_{1}, \ldots, x_{n+k}=e_{k}\right\},
$$

где $n \in \mathbb{Z}, k \in \mathbb{N}, e_{1} \in \mathscr{E}\left(\Gamma_{n+1}\right), \ldots, e_{k} \in \mathscr{E}\left(\Gamma_{n+k}\right)$ и $F\left(e_{i}\right)=I\left(e_{i+1}\right)$. Семейство всех цилиндров также является полукольцом, которое мы обозначим $\mathfrak{C}$.

1.1.3. Конечно аддитивные меры. Пусть $\mathfrak{V}^{+}(X)-$ множество конечно аддитивных вещественнозначных мер $\Phi^{+}$, определенных на полукольце $\mathfrak{C}^{+}$ и таких, что для любых $x, x^{\prime} \in X$, для которых $F\left(x_{n}\right)=F\left(x_{n}^{\prime}\right)$, выполнено равенство $\Phi^{+}\left(\gamma_{n}^{+}(x)\right)=\Phi^{+}\left(\gamma_{n}^{+}\left(x^{\prime}\right)\right)$.

Зафиксируем некоторую меру $\Phi^{+} \in \mathfrak{V}^{+}(X)$. Возьмем $l \in \mathbb{Z}$ и выберем $m$ точек $x(1), x(2), \ldots, x(m) \in X$, для которых

$$
F\left((x(i))_{l+1}\right)=i, \quad i=1, \ldots, m .
$$

Определим вектор $v^{(l)} \in \mathbb{R}^{m}$ по формуле

$$
\left(v^{(l)}\right)_{i}=\Phi^{+}\left(\gamma_{l+1}^{+}(x(i))\right) .
$$

По определению, векторы $v^{(l)}$ удовлетворяют соотношению

$$
v^{(l+1)}=A_{l} v^{(l)}, \quad l \in \mathbb{Z} .
$$

Последовательность $v^{(l)}, l \in \mathbb{Z}$, векторов с вещественными координатами, удовлетворяющих (1), будем называть эквивариантной последовательностъю (относительно последовательности матриц $A_{l}$ или, как мы иногда будем говорить, относительно марковского компакта $X$ ). 
Таким образом, каждой конечно аддитивной мере $\Phi^{+} \in \mathfrak{V}^{+}(X)$ однозначно сопоставляется эквивариантная последовательность $v^{(l)}, l \in \mathbb{Z}$. Определим отображение

$$
\mathrm{eval}_{0}^{+}: \mathfrak{V}^{+}(X) \rightarrow \mathbb{R}^{m}
$$

сопоставляющее каждой мере нулевой элемент соответствующей последовательности:

$$
\operatorname{eval}_{0}^{+}\left(\Phi^{+}\right)=v^{(0)}
$$

Обратно, каждой эквивариантной последовательности $\mathbf{v}=\left(v^{(l)}\right), l \in \mathbb{Z}$, поставим в соответствие конечно аддитивную меру $\Phi_{\mathbf{v}}^{+} \in \mathfrak{V}^{+}(X)$ такую, что если $x \in X$ и $n \in \mathbb{Z}$, то

$$
\Phi_{\mathbf{v}}^{+}\left(\gamma_{n+1}^{+}(x)\right)=\left(v^{(n)}\right)_{F\left(x_{n+1}\right)} .
$$

Отметим, что если все матрицы $A_{n}, n \in \mathbb{Z}$, обратимы, то отображение $\mathrm{eval}_{0}^{+}$ является изоморфизмом.

Аналогичным образом, обозначим $\mathfrak{V}^{-}(X)$ пространство конечно аддитивных вещественнозначных мер $\Phi^{-}$на полукольце $\mathfrak{C}$ таких, что если $I\left(x_{n}\right)=I\left(x_{n}^{\prime}\right)$, то

$$
\Phi^{-}\left(\gamma_{n}^{-}(x)\right)=\Phi^{-}\left(\gamma_{n}^{-}\left(x^{\prime}\right)\right)
$$

Взяв некоторое $l \in \mathbb{Z}$ и выбрав $m$ точек $x(1), x(2), \ldots, x(m) \in X$ так, чтобы

$$
I\left((x(i))_{l}\right)=i, \quad i=1, \ldots, m,
$$

сопоставим мере $\Phi^{-} \in \mathfrak{V}^{-}(X)$ вектор $\widetilde{v}^{(l)}$ по формуле

$$
\left(\widetilde{v}^{(l)}\right)_{i}=\Phi^{-}\left(\gamma_{l}^{-}(x(i))\right) .
$$

По определению, векторы $\widetilde{v}^{(l)}$ удовлетворяют соотношению

$$
\widetilde{v}^{(l)}=A_{l}^{t} \widetilde{v}^{(l+1)}, \quad l \in \mathbb{Z} .
$$

Последовательность $\widetilde{v}^{(l)}, l \in \mathbb{Z}$, векторов с вещественными координатами, удовлетворяющих (2), будем называть обратно эквивариантной (относительно последовательности $A_{l}$ или марковского компакта $\left.X\right)$.

Каждой конечно аддитивной мере $\Phi^{-} \in \mathfrak{V}^{-}(X)$ однозначно сопоставляется обратно эквивариантная последовательность $\widetilde{v}^{(l)}, l \in \mathbb{Z}$. Отображение

$$
\operatorname{eval}_{0}^{-}: \mathfrak{V}^{-}(X) \rightarrow \mathbb{R}^{m}
$$

сопоставляет мере нулевой элемент соответствующей ей последовательности:

$$
\operatorname{eval}_{0}^{-}\left(\Phi^{-}\right)=\widetilde{v}^{(0)}
$$

И обратно, каждой обратно эквивариантной последовательности $\widetilde{\mathbf{v}}=\left(\widetilde{v}^{(l)}\right)$, $l \in \mathbb{Z}$, поставим в соответствие меру $\Phi_{\widetilde{\mathbf{v}}}^{-}$такую, что если $x \in X$ и $n \in \mathbb{Z}$, то

$$
\Phi_{\widetilde{\mathbf{v}}}^{-}\left(\gamma_{n}^{-}(x)\right)=\left(v^{(n)}\right)_{I\left(x_{n}\right)} .
$$

Если все матрицы $A_{l}, l \in \mathbb{Z}$, обратимы, то eval ${ }_{0}^{-}$является изоморфизмом. 
Определим следующие два подпространства конечно аддитивных мер из $\mathfrak{V}^{+}(X)$, обладающих дополнительными свойствами непрерывности.

Пусть

$$
\begin{aligned}
\mathfrak{B}_{c}^{+}(X)= & \left\{\Phi^{+} \in \mathfrak{V}^{+}(X): \lim _{n \rightarrow \infty} \max _{x \in X}\left|\Phi^{+}\left(\gamma_{-n}^{+}(x)\right)\right|=0\right\}, \\
\mathfrak{B}^{+}(X)= & \left\{\Phi^{+} \in \mathfrak{V}^{+}(X): \text { существуют } \alpha>0, C>0\right. \\
& \text { такие, что } \left.\max _{x \in X}\left|\Phi^{+}\left(\gamma_{-n}^{+}(x)\right)\right| \leqslant C e^{-\alpha n} \text { при всех } n \geqslant 0\right\} .
\end{aligned}
$$

ЗАМЕЧАНИЕ. В силу голономной инвариантности максимум в обоих определениях берется по конечному множеству значений.

Аналогично,

$$
\begin{aligned}
\mathfrak{B}_{c}^{-}(X)= & \left\{\Phi^{-} \in \mathfrak{V}^{-}(X): \lim _{n \rightarrow \infty} \max _{x \in X}\left|\Phi^{-}\left(\gamma_{n}^{-}(x)\right)\right|=0\right\}, \\
\mathfrak{B}^{-}(X)= & \left\{\Phi^{-} \in \mathfrak{V}^{-}(X): \text { существуют } \alpha>0, C>0\right. \\
& \text { такие, что } \left.\max _{x \in X}\left|\Phi^{-}\left(\gamma_{n}^{-}(x)\right)\right| \leqslant C e^{-\alpha n} \text { при всех } n \geqslant 0\right\} .
\end{aligned}
$$

ЗАмЕчАниЕ. В контексте слоений на двумерных поверхностях оценку для числа инвариантных мер дал А. Б. Каток [28], а конечно аддитивные трансверсальные инвариантные меры рассматривал Ф. Бонахон [8], [9].

1.1.4. Меры прямого произведения и двойственность. Определим для мер $\Phi^{+} \in \mathfrak{V}^{+}(X), \Phi^{-} \in \mathfrak{V}^{-}(X)$ меру $\Phi^{+} \times \Phi^{-}$на полукольце $\mathfrak{C}(X)$ цилиндров на $X$ следующим образом: для цилиндра $C \in \mathfrak{C}$ и точки $x \in C$ положим

$$
\Phi^{+} \times \Phi^{-}(C)=\Phi^{+}\left(\gamma_{\infty}^{+}(x) \cap C\right) \cdot \Phi^{-}\left(\gamma_{\infty}^{-}(x) \cap C\right) .
$$

Заметим, что в силу голономной инвариантности мер $\Phi^{+}$и $\Phi^{-}$выражение в правой части не зависит от выбора точки $x \in C$ и потому левая часть равенства корректно определена.

Зададим спаривание $\langle\cdot, \cdot\rangle$ между пространствами $\mathfrak{V}^{+}(X)$ и $\mathfrak{V}^{-}(X)$ формулой

$$
\left\langle\Phi^{+}, \Phi^{-}\right\rangle=\Phi^{+} \times \Phi^{-}(X)
$$

Если $\mathbf{v}=\left(v^{(n)}\right)$ - эквивариантная последовательность, соответствующая $\Phi^{+}$, а $\widetilde{\mathbf{v}}=\left(\widetilde{v}^{(n)}\right)$ - обратно эквивариантная последовательность, соответствующая $\Phi^{-}$, то ясно, что

$$
\left\langle\Phi^{+}, \Phi^{-}\right\rangle=\sum_{i=1}^{m} v_{i}^{(0)} \widetilde{v}_{i}^{(0)} .
$$

В частности, если все матрицы $A_{n}, n \in \mathbb{Z}$, обратимы, то спаривание (3) невырождено в ограничении на пару подпространств $\mathfrak{V}^{+}(X), \mathfrak{V}^{-}(X)$. 
1.1.5. Строгая эргодичность. Предположим, что каждое из пространств $\mathfrak{V}^{+}(X), \mathfrak{V}^{-}(X)$ содержит лишь одну положительную меру с точностью до умножения на скаляр. Более того, предположим, что положительная мера $\nu^{+} \in \mathfrak{V}^{+}(X)$ удовлетворяет условиям

$$
\lim _{n \rightarrow \infty} \max _{x \in X} \nu^{+}\left(\gamma_{-n}^{+}(x)\right)=0, \quad \lim _{n \rightarrow \infty} \min _{x \in X} \nu^{+}\left(\gamma_{n}^{+}(x)\right)=\infty,
$$

а положительная мера $\nu^{-} \in \mathfrak{V}^{-}(X)-$ условиям

$$
\lim _{n \rightarrow \infty} \max _{x \in X} \nu^{-}\left(\gamma_{n}^{-}(x)\right)=0, \quad \lim _{n \rightarrow \infty} \min _{x \in X} \nu^{-}\left(\gamma_{-n}^{-}(x)\right)=\infty .
$$

Тогда марковский компакт $X$ называется строго эргодическим. Равносильное определение строгой эргодичности дается следующим условием, восходящим к Г. Фюрстенбергу (см., например, формулу (16.13) в [24]).

УСловие 1.1. 1. Для любого $l \in \mathbb{Z}$ существует такой вектор

$$
\lambda^{(l)}=\left(\lambda_{1}^{(l)}, \ldots, \lambda_{m}^{(l)}\right),
$$

все координаты которого положительны, что $\lambda^{(l)}=A_{l}^{t} \lambda^{(l+1)} u$

$$
\bigcap_{n \in \mathbb{N}} A_{l+1}^{t} \cdots A_{l+n}^{t} \mathbb{R}_{+}^{m}=\mathbb{R}_{+} \lambda^{(l)} .
$$

2. Для любого $l \in \mathbb{Z}$ существует такой вектор

$$
h^{(l)}=\left(h_{1}^{(l)}, \ldots, h_{m}^{(l)}\right),
$$

все координаты которого положительны, что $h^{(l)}=A_{l} h^{(l-1)} u$

$$
\bigcap_{n \in \mathbb{N}} A_{l-1} \cdots A_{l-n} \mathbb{R}_{+}^{m}=\mathbb{R}_{+} h^{(l)} .
$$

3. $\left|\lambda^{(l)}\right| \rightarrow 0$ nрu $l \rightarrow \infty,\left|h^{(l)}\right| \rightarrow 0$ npu $l \rightarrow-\infty$.

4. $\min _{i} \lambda_{i}^{(l)} \rightarrow \infty$ npu $l \rightarrow-\infty, \min _{i} h_{i}^{(l)} \rightarrow \infty$ npu $l \rightarrow \infty$.

Последовательности векторов $\lambda^{(l)}$ и $h^{(l)}$ определены с точностью до умножения на константу (не зависящую от $l$ ). Последовательность $\left(h^{(l)}\right)$ является эквивариантной, а последовательность $\left(\lambda^{(l)}\right)$ - обратно эквивариантной.

Для нормировки векторов $\left(\lambda^{(l)}\right),\left(h^{(l)}\right)$, задаваемых в условии 1.1, будем требовать выполнения равенств

$$
\left|\lambda^{(0)}\right|=1, \quad\left\langle\lambda^{(0)}, h^{(0)}\right\rangle=1 .
$$

Тогда в силу эквивариантности

$$
\left\langle\lambda^{(l)}, h^{(l)}\right\rangle=1 \quad \text { для всех } l \in \mathbb{Z} .
$$

Пусть $\nu_{X}^{+}-$мера, соответствующая эквивариантной последовательности $h^{(l)}$. Заметим, что тогда $\nu_{X}^{+}-$положительная $\sigma$-конечная $\sigma$-аддитивная мера на $\sigma$-алгебре, порождаемой полукольцом $\mathfrak{C}^{+}$. Аналогично, мера $\nu_{X}^{-}$, соответствующая обратно эквивариантной последовательности $\lambda^{(l)}$, является положительной $\sigma$-конечной $\sigma$-аддитивной мерой на $\sigma$-алгебре, порождаемой $\mathfrak{C}^{-}$. Определим, наконец, вероятностную меру $\nu_{X}$ на $X$ по формуле

$$
\nu_{X}=\nu_{X}^{+} \times \nu_{X}^{-}
$$


1.1.6. Слабо липшицевы функции. Пусть $X$ - строго эргодический марковский компакт. Определим пространство $\operatorname{Lip}_{w}^{+}(X)$ слабо липшицевых функиий на нем следующим образом. Ограниченная борелевская функция $f: X \rightarrow \mathbb{R}$ является слабо липшицевой, если существует такая константа $C>0$, что при всех $n \geqslant 0$ и любых $x, x^{\prime} \in X$, для которых $F\left(x_{n+1}\right)=F\left(x_{n+1}^{\prime}\right)$, выполнено неравенство

$$
\left|\int_{\gamma_{n}^{+}(x)} f d \nu^{+}-\int_{\gamma_{n}^{+}\left(x^{\prime}\right)} f d \nu^{+}\right| \leqslant C .
$$

Если $C_{f}$ - точная нижняя грань множества значений $C$, для которых верно (4), то норма функции $f \in \operatorname{Lip}_{w}^{+}(X)$ равна

$$
\|f\|_{\operatorname{Lip}_{w}^{+}}=\sup _{X}|f|+C_{f} .
$$

Функции, интеграл которых по мере $\nu$ равен нулю, образуют в $\operatorname{Lip}_{w}^{+}(X)$ подпространство, которое мы обозначим $\operatorname{Lip}_{w, 0}^{+}(X)$.

1.1.7. Упорядочения Вершика. Роль упорядочений Вершика для символического кодирования потоков на плоских поверхностях, неформально говоря, заключается в следующем. Марковский компакт и его асимптотические слоения соответствуют плоской поверхности и вертикальному и горизонтальному слоениям на ней. Однако, чтобы задать сами потоки переноса вдоль этих слоений, нужно ввести линейный порядок на листах слоений. Этот линейных порядок порождается упорядочением Вершика на ребрах графов, задающих марковский компакт.

Перейдем к формальным определениям.

Пусть $Г \in \mathfrak{G}$. Следуя С. Ито [26] и А. М. Вершику [44], [45], предположим, что для каждого $i \in\{1, \ldots, m\}$ задан линейный порядок на множестве

$$
\{e \in \mathscr{E}(\Gamma): I(e)=i\} .
$$

Такой набор порядков называют упорядочением Вериика на Г.

Пусть $X$ - строго эргодический марковский компакт, соответствующий последовательности графов $\Gamma_{l}$.

Если на каждом из графов $\Gamma_{l}, l \in \mathbb{Z}$, задано упорядочение Вершика, то на каждом листе слоения $\mathscr{F}_{X}^{+}$индуцируется линейный порядок. Действительно, если $x^{\prime} \in \gamma_{\infty}^{+}(x), x^{\prime} \neq x$, то существует такое $n$, что $x_{t}=x_{t}^{\prime}$ при $t>n$, но $x_{n} \neq x_{n}^{\prime}$. Поскольку $I\left(x_{n}\right)=I\left(x_{n}^{\prime}\right)$, ребра $x_{n}$ и $x_{n}^{\prime}$ сравнимы в смысле данного упорядочения; если $x_{n}<x_{n}^{\prime}$, то мы полагаем $x<x^{\prime}$. Этот набор упорядочений Вершика на графах $\Gamma_{l}, l \in \mathbb{Z}$, мы будем называть упорядочением Вершика на марковском компакте $X$ и обозначать через $\mathfrak{o}$.

Ребро называется максимальным (относительно o), если не существует большего ребра, и минимальным, если не существует меньшего ребра; ребро $е$ называется следующим за ребром $e^{\prime}$, если $e>e^{\prime}$, но не существует ребра $e^{\prime \prime}$, для которого $e>e^{\prime \prime}>e^{\prime}$. Замкнутым интервалом $\left[x, x^{\prime}\right]$ будем называть множество точек $x^{\prime \prime}$, удовлетворяющих неравенству $x \leqslant x^{\prime \prime} \leqslant x^{\prime}$, а открьтым интервалом $\left(x, x^{\prime}\right)$ - множество точек $x^{\prime \prime}$, удовлетворяющих неравенству $x<x^{\prime \prime}<x^{\prime}$. 


\section{2. Случайные марковские компакты}

1.2.1. Пространство марковских компактов. Напомним, что $\mathfrak{G}$ - это множество ориентированных графов с $m$ вершинами, возможно с кратными ребрами, причем из каждой вершины исходит хотя бы одно ребро и в каждую вершину входит как минимум одно ребро.

Пусть $\Omega$ - это пространство бесконечных в обе стороны последовательностей графов $\Gamma_{n} \in \mathfrak{G}$ :

$$
\Omega=\left\{\omega=\ldots \omega_{-n} \ldots \omega_{n} \ldots, \omega_{i} \in \mathfrak{G}, i \in \mathbb{Z}\right\} .
$$

Будем обозначать $X(\omega)$ марковский компакт, соответствующий последовательности $\omega \in \Omega$.

Как указано в предыдущем разделе, при любом $\omega$ на марковском компакте $X(\omega)$ заданы слоения $\mathscr{F}^{+}, \mathscr{F}^{-}$, полукольца $\mathfrak{C}^{+}, \mathfrak{C}^{-}$и т. д.; чтобы подчеркнуть зависимость от $\omega$, мы будем обозначать их $\mathscr{F}_{\omega}^{+}, \mathfrak{C}_{\omega}^{+}$и т. д.

Левый сдвиг $\sigma$ на пространстве $\Omega$ определяется как $(\sigma \omega)_{n}=\omega_{n+1}$.

1.2.2. Ренормализационный коцикл. Имеется естественный коцикл $\mathbb{A}$ над динамической системой $(\Omega, \sigma)$, определяемый при $n>0$ формулой

$$
\mathbb{A}(n, \omega)=A\left(\omega_{n}\right) \cdots A\left(\omega_{1}\right) .
$$

Коцикл $\mathbb{A}$ называется ренормализационным коциклом.

Пусть подмножество $\Omega_{\text {inv }} \subset \Omega$ состоит из всех тех последовательностей $\omega$, для которых все матрицы $A\left(\omega_{n}\right)$ обратимы.

При $\omega \in \Omega_{\text {inv }}$ и $n<0$ положим

$$
\mathbb{A}(n, \omega)=A^{-1}\left(\omega_{-n}\right) \cdots A^{-1}\left(\omega_{0}\right)
$$

пусть также $\mathbb{A}(0, \omega)$ - единичная матрица.

Пусть $\mu$ - эргодическая $\sigma$-инвариантная вероятностная мера на $\Omega$, удовлетворяющая следующему условию.

Условие 1.2. 1. Существует такое $\Gamma_{0} \in \mathfrak{G}$, что все элементы матрииъь $A\left(\Gamma_{0}\right)$ положительны и

$$
\mu\left(\left\{\omega: \omega_{0}=\Gamma_{0}\right\}\right)>0 .
$$

2. Матрицы $A\left(\omega_{n}\right)$ обратимы для почти всех $\omega$ относителъно меры $\mu$.

3. Логарифм нормы ренормализачионного кочикла, а также обратного кнему интегрируем по мере $\mu$.

Из этих условий на меру $\mu$ вытекает существование матрицы $Q$, все элементы которой положительны и которая входит бесконечно много раз в последовательность матриц $A\left(\omega_{n}\right), n \in \mathbb{Z}$, как в будущем, так и в прошлом для $\mu$-почти всех $\omega \in \Omega$. Отсюда следует, что для $\mu$-почти всех $\omega \in \Omega$ марковский компакт $X(\omega)$ строго эргодичен.

Более того, пусть $E_{\omega}^{u}$ обозначает для $\mu$-почти всех $\omega \in \Omega$ строго неустойчивое оселедцевское подпространство ренормализационного коцикла $\mathbb{A}$ в точке $\omega$. 
ПреДЛОЖЕНИЕ 1.3. Для $\mu$-почти всех $\omega \in \Omega$ отображение $\mathrm{eval}_{0}^{+}$индуцирует изоморфизм между пространствами $\mathfrak{B}^{+}(X(\omega))$ и $E_{\omega}^{u}$.

ДокАЗАТЕЛЬство. Это прямое следствие мультипликативной эргодической теоремы Оселедца (см. [5] и приложение А).

Предположим теперь, что для $\mu$-почти всех $\omega \in \Omega$ задано упорядочение Вершика $\mathfrak{o}(\omega)$ на ребрах каждого из графов $\omega_{n}, n \in \mathbb{Z}$; при этом предполагается, что упорядочение $\mathfrak{o}(\omega)$ является $\sigma$-инвариантным в том смысле, что упорядочение $\mathfrak{o}(\omega)$ на ребрах графа $\omega_{n+1}$ совпадает с упорядочением $\mathfrak{o}(\sigma \omega)$ на ребрах графа $(\sigma \omega)_{n}=\omega_{n+1}$.

Как и раньше, на листах слоения $\mathscr{F}_{\omega}^{+}$упорядочение $\mathfrak{o}(\omega)$ определяет линейный порядок, $\left[x, x^{\prime}\right]$ и $\left(x, x^{\prime}\right)$ обозначают соответственно замкнутый и открытый интервал относительно этого линейного порядка и т. д.

1.2.3. Приближение слабо липшицевых функций. Для $\omega \in \Omega$ будем обозначать $\mathfrak{C}_{\omega}^{+}(\mathfrak{o})$ полукольцо дуг вида $\left[x, x^{\prime}\right),\left(x, x^{\prime}\right],\left[x, x^{\prime}\right],\left(x, x^{\prime}\right)$. По определению, $\mathfrak{C}_{\omega}^{+}(\mathfrak{o}) \supset \mathfrak{C}_{\omega}^{+}$.

ПРеДЛОЖЕНИЕ 1.4. Для $\mu$-почти всех $\omega \in \Omega$ любую конечно аддитивную меру $\Phi^{+} \in \mathfrak{B}^{+}(X(\omega))$ можно продолжить, и притом единственным образом, на полукольио $\mathfrak{C}^{+}(\mathfrak{o})_{\omega}$ так, что функция $\Phi^{+}\left(\left[x^{\prime}, x^{\prime \prime}\right)\right)$ является непрерывной no $x^{\prime}$ u no $x^{\prime \prime}$.

ПРЕДЛОЖЕНИЕ 1.5. Для любого $\varepsilon>0$ существует положительная измеримая функиия $C_{\varepsilon}: \Omega \rightarrow \mathbb{R}_{>0}$, для которой выполнено следующее. При $\mu$-почти всех $\omega \in \Omega$ существует такое непрерывное отображение

$$
\Xi_{\omega}^{+}: \operatorname{Lip}_{w}^{+}(X(\omega)) \rightarrow \mathfrak{B}^{+}(X(\omega)),
$$

что для любой $f \in \operatorname{Lip}_{w}^{+}(X(\omega))$ и любых $x^{\prime}, x^{\prime \prime} \in X(\omega), x^{\prime}<x^{\prime \prime}$, выполнено неравенство

$$
\left|\int_{\left[x^{\prime}, x^{\prime \prime}\right]} f d \nu^{+}-\Xi_{\omega}^{+}\left(f ;\left[x^{\prime}, x^{\prime \prime}\right]\right)\right| \leqslant C_{\varepsilon}(\omega) \cdot\|f\|_{\operatorname{Lip}_{w}^{+}} \cdot\left(1+\nu^{+}\left(\left[x^{\prime}, x^{\prime \prime}\right]\right)\right)^{\varepsilon} .
$$

1.2.4. Транспонированный коцикл и двойственность. Отображение $\Xi_{\omega}^{+}$из предложения 1.5 имеет следующее явное описание в терминах двойственности между гёльдеровскими коциклами, соответствующими прошлому и будущему нашего марковского компакта. Эта двойственность является одной из центральных конструкций в настоящей работе.

ПРеДЛОЖеНИЕ 1.6. Для $\mu$-почти всех $\omega \in \Omega$ спаривание $\langle\cdot, \cdot\rangle$ из $n .1 .1 .4$ невырождено в ограничении на пару подпространств $\mathfrak{B}^{+}(X(\omega))$ и $\mathfrak{B}^{-}(X(\omega))$.

Используя эту двойственность, мы получаем следующее предложение.

ПРЕДЛОЖЕНИЕ 1.7. Для $\mu$-почти всех $\omega \in \Omega$ и любых $f \in \operatorname{Lip}_{w}^{+}(X(\omega))$ и $\Phi^{-} \in \mathfrak{B}^{-}(X(\omega))$ выполнено равенство

$$
\left\langle\Xi_{\omega}^{+}(f), \Phi^{-}\right\rangle=\int_{X(\omega)} f d \nu^{+} \times \Phi^{-} .
$$


В силу предыдущего предложения соотношение (5) определяет $\Xi_{\omega}^{+}(f)$ однозначно.

ДокАЗАТЕЛЬСтво ПРЕДЛОжЕНИЯ 1.6. Так же, как пространство $\mathfrak{B}^{+}(X(\omega))$ отождествляется со строго неустойчивым ляпуновским подпространством ренормализационного коцикла $\mathbb{A}$, пространство $\mathfrak{B}^{-}(X(\omega))$ отождествляется со строго неустойчивым ляпуновским подпространством транспонированного коцикла $\mathbb{A}^{t}$, определяемого следующим образом.

ОПРЕДЕЛЕНИЕ. Транспонированный коцикл $\mathbb{A}^{t}$ над динамической системой $\left(\Omega, \sigma^{-1}, \mathrm{P}\right)$ задается при $n>0$ формулой

$$
\mathbb{A}^{t}(n, \omega)=A^{t}\left(\omega_{1-n}\right) \cdots A^{t}\left(\omega_{0}\right)
$$

Если $\omega \in \Omega_{\mathrm{inv}}$, то при $n<0$ он задается формулой

$$
\mathbb{A}^{t}(n, \omega)=\left(A^{t}\right)^{-1}\left(\omega_{-n}\right) \cdots\left(A^{t}\right)^{-1}\left(\omega_{1}\right)
$$

а матрица $\mathbb{A}^{t}(0, \omega)$ полагается единичной.

Пусть $\widetilde{E}_{\omega}^{u}$ обозначает для $\mu$-почти всех $\omega \in \Omega$ строго неустойчивое оселедцевское подпространство транспонированного коцикла $\mathbb{A}^{t}$ в точке $\omega$.

Тогда из мультипликативной теоремы Оселедца непосредственно вытекает следующее предложение.

ПРЕДЛОЖЕНИЕ 1.8. При $\mu$-почти всех $\omega$ отображение $\mathrm{eval}_{0}^{-}$индуиирует изоморфизм между пространствами $\mathfrak{B}^{-}(X(\omega))$ и $\widetilde{E}_{\omega}^{u}$.

Более того, из мультпликативной эргодической теоремы следует также, что стандартное скалярное произведение в $\mathbb{R}^{m}$ индуцирует при $\mu$-почти всех $\omega \in \Omega$ невырожденное спаривание между подпространствами $E_{\omega}^{u}$ и $\widetilde{E}_{\omega}^{u}$.

Предложение 1.7 будет доказано в следующей главе.

В следующей главе мы построим специальный поток $h_{t}^{+}$на строго эргодичном марковском компакте $X$ с упорядочением Вершика о такой, что дуги потока $h_{t}^{+}$являются интервалами вида $\left[x^{\prime}, x^{\prime \prime}\right]$. Тогда из предложения 1.5 будет получаться асимптотическое разложение эргодического интеграла для таких символических потоков.

Левый сдвиг $\sigma$ на пространстве $\Omega$ марковских компактов естественным образом сплетает пространства $\mathfrak{B}_{\omega}^{ \pm}$и $\mathfrak{B}_{\sigma \omega}^{ \pm}$; свойства действия сдвига на соответствующих эквивариантных и обратно эквивариантных последовательностях определяются мультипликативной эргодической теоремой Оселедца, примененной к коциклам $\mathbb{A}, \mathbb{A}^{t}$. Это ренормализационное действие сдвига будет играть ключевую роль в доказательстве предельных теорем для рассматриваемых символических потоков и, следовательно, для потоков переноса. 


\section{Глава 2. Символическая аппроксимационная теорема}

\section{1. Подготовительная аппроксимационная лемма}

2.1.1. Случай одностороннего марковского компакта. Для нашей первой аппроксимационной леммы нужна структура только одностороннего марковского компакта.

Как и раньше, по односторонней бесконечной последовательности графов $\Gamma_{n} \in \mathfrak{G}, n \in \mathbb{N}$, мы строим односторонний марковский компакт

$$
Y=\left\{y=y_{1} \ldots y_{n} \ldots, y_{n} \in \mathscr{E}\left(\Gamma_{n}\right), I\left(y_{n}\right)=F\left(y_{n+1}\right)\right\}
$$

и обозначаем для $y \in Y$

$$
\gamma_{n}^{+}(y)=\left\{y^{\prime} \in Y: y_{t}^{\prime}=y_{t} \text { при } t \geqslant n\right\}, \quad \gamma_{\infty}^{+}(y)=\bigcup_{n=0}^{\infty} \gamma_{n}^{+}(y) .
$$

Отметим, что множества $\gamma_{n}^{+}(y)$ конечны, а множество $\gamma_{\infty}^{+}(y)$ счетно.

Пусть $\operatorname{Lip}_{w}^{+}(Y)$ - пространство функций $\varphi: Y \rightarrow \mathbb{R}$, для которых существует константа $C>0$ такая, что для любого $n \in \mathbb{N}$ и любых $y^{\prime}, y^{\prime \prime} \in Y$, для которых $F\left(y_{n}^{\prime}\right)=F\left(y_{n}^{\prime \prime}\right)$, выполнено неравенство

$$
\left|\sum_{y \in \gamma_{n}^{+}\left(y^{\prime}\right)} \varphi(y)-\sum_{y \in \gamma_{n}^{+}\left(y^{\prime \prime}\right)} \varphi(y)\right| \leqslant C .
$$

Пусть $C_{\varphi}-$ наименьшая константа, для которой справедлива последняя формула, тогда норма на пространстве $\operatorname{Lip}_{w}^{+}(Y)$ задается формулой

$$
\|\varphi\|_{\operatorname{Lip}_{w}^{+}}=C_{\varphi}+\sup _{y \in Y}|\varphi(y)| .
$$

Частным случаем слабо липшицевых функций являются кусочно постоянные функиии, определяемые следующим образом.

Для вектора $v \in \mathbb{R}^{m}, v=\left(v_{1}, \ldots, v_{m}\right)$, положим

$$
\Phi_{v}(y)=v_{F\left(y_{1}\right)}
$$

Следующая лемма утверждает, что суммы слабо липшицевых функций можно приблизить кусочно постоянными функциями с точностью до субэкспоненциальной ошибки. Нам понадобится следующее условие на матрицы $A_{n}, n \geqslant 0$.

УСловие 2.1. Для каждого $n \geqslant 1$ имеются разложения в прямую сумму

$$
\mathbb{R}^{m}=E_{n}^{u} \oplus E_{n}^{c s},
$$

которые удовлетворяют следующим условиям:

1) $A_{n} E_{n}^{u}=E_{n+1}^{u}$ и $\left.A_{n}\right|_{E_{n}^{u}}$ инбективно;

2) $A_{n} E_{n}^{c s} \subset E_{n+1}^{c s}$; 
3) для любого $\varepsilon>0$ существует такое $C_{\varepsilon}>0$, что при всех $n \geqslant 1, k \geqslant 0$ выполнены неравенства

$$
\begin{array}{r}
\left\|\left.\left(A_{n+k} \cdots A_{n}\right)^{-1}\right|_{E_{n+k+1}^{u}}\right\| \leqslant C_{\varepsilon} e^{\varepsilon n-\alpha k}, \\
\left\|\left.A_{n+k} \cdots A_{n}\right|_{E_{n}^{c s}}\right\| \leqslant C_{\varepsilon} e^{\varepsilon(n+k)}
\end{array}
$$

с некоторим $\alpha>0$.

Лемма 2.2. Пусть $Y$ - односторонний марковский компакт, для которого матрици $A_{n}, n \geqslant 1$, удовлетворяют условию 2.1. Тогда существует непрерывное отображение

$$
\Xi^{+}: \operatorname{Lip}_{w}^{+}(Y) \rightarrow E_{0}^{u}
$$

такое, что для любого $\varepsilon>0$ существует константа $\widetilde{C}_{\varepsilon}$ такая, что для любой $\varphi \in \operatorname{Lip}_{w}^{+}(Y)$, любого $n \in \mathbb{N}$ и любого $y^{\prime} \in Y$ выполнено неравенство

$$
\left|\sum_{y \in \gamma_{n}^{+}\left(y^{\prime}\right)}\left(\varphi(y)-\Phi_{\Xi^{+}(\varphi)}(y)\right)\right| \leqslant \widetilde{C}_{\varepsilon}\|\varphi\|_{\operatorname{Lip}_{w}^{+}} e^{\varepsilon n} .
$$

Сначала мы докажем вспомогательное утверждение.

Лемма 2.3. Пусть последовательность матрии, $A_{n}$ удовлетворяет условию 2.1. Если последовательность векторов $v_{1}, \ldots$ такова, что для любого $\varepsilon>0$ существует константа $C_{\varepsilon}$, для которой при всех $n$ выполнено неравенство

$$
\left|A_{n} v_{n}-v_{n+1}\right| \leqslant C_{\varepsilon} e^{\varepsilon n},
$$

то существует единственный вектор $v \in E_{1}^{u}$ такой, что

$$
\left|A_{n} \cdots A_{1} v-v_{n+1}\right| \leqslant C_{\varepsilon}^{\prime} e^{\varepsilon n} .
$$

ДокАЗАТЕЛЬСтво. Определим векторы $u_{n+1}=v_{n+1}-A_{n} v_{n}$ и разложим их: $u_{n+1}=u_{n+1}^{+}+u_{n+1}^{-}$, где $u_{n+1}^{+} \in E_{n+1}^{u}, u_{n+1}^{-} \in E_{n+1}^{c s}$. Пусть

$$
\begin{aligned}
& v_{n+1}^{+}=u_{n+1}^{+}+A_{n} u_{n}^{+}+A_{n} A_{n-1} u_{n-1}^{+}+\cdots+A_{n} \cdots A_{1} u_{1}^{+}, \\
& v_{n+1}^{-}=u_{n+1}^{-}+A_{n} u_{n}^{-}+A_{n} A_{n-1} u_{n-1}^{-}+\cdots+A_{n} \cdots A_{1} u_{1}^{-} .
\end{aligned}
$$

Тогда $v_{n+1}^{+} \in E_{n+1}^{u}, v_{n+1}^{-} \in E_{n+1}^{c s}, v_{n+1}=v_{n+1}^{+}+v_{n+1}^{-}$. Рассмотрим теперь вектор

$$
v=u_{1}^{+}+A_{1}^{-1} u_{1}^{+}+\cdots+\left(A_{n} \cdots A_{1}\right)^{-1} u_{n+1}^{+}+\cdots .
$$

По условиям леммы ряд в правой части сходится экспоненциально быстро и, более того, верна оценка

$$
\left|A_{n} \cdots A_{1} v-v_{n+1}^{+}\right| \leqslant C_{\varepsilon}^{\prime} e^{\varepsilon n},
$$

где $C_{\varepsilon}^{\prime}-$ некоторая константа.

Заметим, что по условию $\left|v_{n+1}^{-}\right| \leqslant C_{\varepsilon} e^{\varepsilon n}$. Таким образом, построенный вектор $v$ удовлетворяет заключению леммы. 
Единственность такого вектора $v$ следует из того, что по нашим предположениям для любого $\widetilde{v} \neq 0, \widetilde{v} \in E_{1}^{u}$, выполнено неравенство

$$
\left|A_{n} \cdots A_{1} \widetilde{v}\right| \geqslant C^{\prime \prime} e^{\alpha n} .
$$

Лемма 2.3 доказана.

Перейдем к доказательству леммы 2.2.

Для $i=1, \ldots, m$ и $n \geqslant 1$ зафиксируем произвольные точки $y(n, i) \in Y$, для которых

$$
F\left(y(n, i)_{n}\right)=i
$$

Зададим последовательность векторов $v(n) \in \mathbb{R}^{m}$ формулой

$$
(v(n))_{i}=\sum_{y \in \gamma_{n+1}^{+}(y(n, i))} \varphi(y) .
$$

Так как $\varphi$ липшицева, то

$$
\left|\sum_{\widetilde{y} \in \gamma_{n+1}^{+}(y)} \varphi(\widetilde{y})-v(n)_{F\left(y_{1}\right)}\right| \leqslant C \quad \text { для любой } y \in Y,
$$

откуда по аддитивности следует, что

$$
\left|A_{n} v(n)-v(n+1)\right| \leqslant C_{\varepsilon} e^{\varepsilon n} .
$$

Лемма 2.2 следует теперь из леммы 2.3 .

2.1.2. Двойственность. Вернемся к двусторонним марковским компактам. Пусть $X$ - марковский компакт, соответствующий двусторонней бесконечной последовательности графов $\left(\Gamma_{n}\right)_{n \in \mathbb{Z}}$, а $Y$ - односторонний марковский компакт, отвечающий ее положительной части $\left(\Gamma_{n}\right)_{n \geqslant 1}$.

Тогда имеется естественное забывающее отображение $\Pi_{Y}^{X}$, которое ставит в соответствие двустороннему пути $\left(x_{n}\right)_{n \in \mathbb{Z}}$ его положительную часть $\left(x_{n}\right)_{n \geqslant 1}$.

Будем называть конечно аддитивную меру $\Theta$ на полукольце $\mathfrak{C}_{0}^{+}(X)$ слабо липшицевой, если функция $\varphi_{\Theta}: Y \rightarrow \mathbb{R}$, определяемая как

$$
\varphi_{\Theta}(y)=\Theta\left(\gamma_{1}^{+}(x)\right), \quad x \in\left(\Pi_{Y}^{X}\right)^{-1} y,
$$

слабо липшицева.

Отметим, что функция $\varphi_{\Theta}$ корректно определена, поскольку множество $\gamma_{1}^{+}(x)$ не зависит от конкретного выбора $x \in\left(\Pi_{Y}^{X}\right)^{-1} y$. Множество слабо липшицевых мер на $X$ обозначим через $\operatorname{LipMeas}^{+}(X)$.

Отображение $\Theta \mapsto \varphi_{\Theta}$ взаимно однозначно отображает $\operatorname{LipMeas}^{+}(X)$ на $\operatorname{Lip}_{w}^{+}(Y)$, так что мы можем задать норму на пространстве LipMeas ${ }^{+}(X)$ формулой

$$
\|\Theta\|_{\text {LipMeas }^{+}}=\|\varphi\|_{\text {Lip }_{w}^{+}} .
$$

Зададим спаривание элементов пространств $\operatorname{LipMeas}^{+}(X)$ и $\mathfrak{B}^{-}(X)$ следующим образом. Пусть $\Theta \in \operatorname{LipMeas}^{+}(X)$, а $\Phi^{-} \in \mathfrak{B}^{-}(X)$ задана обратно эквивариантной последовательностью векторов $\widetilde{v}^{(n)}, n \in \mathbb{Z}$. 
Выберем для каждого $n \in \mathbb{N}$ точки $x^{(n)}(1), \ldots, x^{(n)}(m) \in X$, для которых

$$
F\left(\left(x^{(n)}(i)\right)_{n}\right)=i, \quad i=1, \ldots, m .
$$

Рассмотрим сумму Римана

$$
S_{\mathrm{Riem}}\left(\Theta, \Phi^{-}, x^{(n)}(1), \ldots, x^{(n)}(m)\right)=\sum_{i=1}^{m} \Theta\left(\gamma_{n}^{+}\left(x^{(n)}(i)\right)\right) \cdot \widetilde{v}_{i}^{(n-1)}
$$

и положим

$$
\left\langle\Theta, \Phi^{-}\right\rangle=\lim _{n \rightarrow \infty} S_{\text {Riem }}\left(\Theta, \Phi^{-}, x^{(n)}(1), \ldots, x^{(n)}(m)\right) .
$$

Существование предела и его независимость от конкретного выбора точек $x^{(n)}(1), \ldots, x^{(n)}(m)$ вытекает из условия Липшица для $\Theta$ и экспоненциального убывания норм векторов $\widetilde{v}^{(n)}$. Ясно также, что если $\Theta \in \mathfrak{V}^{+}(X)$, то новое определение спаривания совпадает с данным выше.

Пусть теперь $X$ - двусторонний марковский компакт с матрицами смежности $A_{n}, n \geqslant 0$, удовлетворяющими условию 2.1. Для $\Theta \in \operatorname{LipMeas}^{+}(X)$ зададим вектор $v \in E_{0}^{u}$ равенством

$$
\Phi_{v}=\Xi^{+}\left(\varphi_{\Theta}\right) \text {. }
$$

ЗАмечАниЕ. Далее вместо записи $\Xi^{+}\left(\varphi_{\Theta}\right)$ мы будем использовать обозначение $\Xi^{+}(\Theta)$.

Из определений немедленно вытекает, что для любого такого $\Phi^{-} \in \mathfrak{B}^{-}$верно

$$
\left\langle\Theta, \Phi^{-}\right\rangle=\left\langle\Xi^{+}(\Theta), \Phi^{-}\right\rangle .
$$

Действительно, пусть $\Phi^{-}=\Phi_{\widetilde{\mathbf{v}}}^{-}$, где $\widetilde{\mathbf{v}}=\left(\widetilde{v}^{(n)}\right)$ - обратно эквивариантная последовательность векторов. По определению,

$$
\left\langle\Theta, \Phi^{-}\right\rangle=\sum_{i=1}^{m} \Xi^{+}\left(\Theta, \gamma_{n}^{+}\left(x^{(n)}(i)\right)\right) \cdot \widetilde{v}_{i}^{(n-1)} .
$$

ЗАмЕчАниЕ. Здесь и далее мы используем запись вида $\Xi^{+}\left(\Theta, \gamma_{n}^{+}(x)\right)$ вместо $\Xi^{+}(\Theta)\left(\gamma_{n}^{+}(x)\right)$.

Следовательно, так как $\Phi^{-} \in \mathfrak{B}^{-}$, мы получаем, что

$$
\begin{aligned}
& \left|\left\langle\Theta-\Xi^{+}(\Theta), \Phi^{-}\right\rangle\right| \\
& \quad \leqslant \sum_{i=1}^{m}\left|\Theta\left(\gamma_{n}^{+}\left(x^{(n)}(i)\right)\right)-\Xi^{+}\left(\Theta, \gamma_{n}^{+}\left(x^{(n)}(i)\right)\right)\right| \cdot\left|\widetilde{v}_{i}^{(n-1)}\right| \leqslant e^{-\alpha n}
\end{aligned}
$$

для некоторого положительного $\alpha$, откуда вытекает (6).

В изучении случайных марковских компактов мы сосредоточимся в основном на случае, когда и последовательность матриц $\left(A_{n}\right)_{n \in \mathbb{Z}}$, и последовательность транспонированных матриц $\left(A_{n}^{t}\right)_{n \in \mathbb{Z}}$ обладают разложениями на неустойчивую и центральную часть, похожими на аналогичные разложения из условия 2.1. Более точно, сформулируем следующее условие на последовательность $(m \times m)$-матриц $\left(A_{n}\right)_{n \in \mathbb{Z}}$. 
УСловиЕ 2.4. Для любого $n \in \mathbb{Z}$ имеются разложения в прямую сумму

$$
\mathbb{R}^{m}=E_{n}^{u} \oplus E_{n}^{c s}, \quad \mathbb{R}^{m}=\widetilde{E}_{n}^{u} \oplus \widetilde{E}_{n}^{c s},
$$

для которых выполнено следующее:

1) $A_{n} E_{n}^{u}=E_{n+1}^{u}, A_{n}^{t} \widetilde{E}_{n+1}^{u}=\widetilde{E}_{n}^{u}$, причем $\left.\left.A_{n}\right|_{E_{n}^{u}} u A_{n}^{t}\right|_{\widetilde{E}_{n+1}^{u}}$ инбективнь при всех $n \in \mathbb{Z}$;

2) $A_{n} E_{n}^{c s} \subset E_{n+1}^{c s}, A_{n}^{t} \widetilde{E}_{n+1}^{c s} \subset \widetilde{E}_{n}^{c s}$ nри всех $n \in \mathbb{Z}$;

3) $\widetilde{E}_{n}^{c s}=\operatorname{Ann}\left(E_{n}^{u}\right), \widetilde{E}_{n}^{u}=\operatorname{Ann}\left(E_{n}^{c s}\right)$ nрu всех $n \in \mathbb{Z}$;

4) существует такое $\alpha>0$, что для каждого $\varepsilon>0$ имеется положительная константа $C_{\varepsilon}$, для которой при всех $k \in \mathbb{N} u n \in \mathbb{Z}$ выполнены неравенства

$$
\begin{array}{r}
\left\|\left.\left(A_{n+k} \cdots A_{n}\right)^{-1}\right|_{E_{n+k+1}^{u}}\right\| \leqslant C_{\varepsilon} e^{\varepsilon|n|-\alpha k}, \\
\left\|\left.A_{n+k} \cdots A_{n}\right|_{E_{n}^{c s}}\right\| \leqslant C_{\varepsilon} e^{\varepsilon(|n|+k)}, \\
\left\|\left.\left(A_{n}^{t} \cdots A_{n+k}^{t}\right)^{-1}\right|_{\widetilde{E}_{n}^{u}}\right\| \leqslant C_{\varepsilon} e^{\varepsilon|n|-\alpha k}, \\
\left\|\left.A_{n}^{t} \cdots A_{n+k}^{t}\right|_{\widetilde{E}_{n+k+1}^{c s}}\right\| \leqslant C_{\varepsilon} e^{\varepsilon(|n|+k)} .
\end{array}
$$

Из условия 2.4 следует, что каждому вектору $v \in E_{0}^{u}$ отвечает единственная эквивариантная последовательность $\mathbf{v}$, а следовательно, единственная конечно аддитивная мера $\Phi_{\mathbf{v}}^{+} \in \mathfrak{V}^{+}(X)$, причем $\Phi_{\mathbf{v}}^{+} \in \mathfrak{B}^{+}(X)$. Аналогично, каждому вектору $\widetilde{v} \in \widetilde{E}_{0}^{u}$ отвечает единственная конечно аддитивная мера $\Phi_{\widetilde{\mathbf{v}}}^{-} \in \mathfrak{V}^{-}(X)$, причем $\Phi_{\widetilde{\mathbf{v}}}^{-} \in \mathfrak{B}^{-}(X)$. Наконец, из третьего утверждения в условии 2.4 вытекает, что спаривание $\langle\cdot, \cdot\rangle$ невырождено на паре подпространств $\mathfrak{B}^{+}(X), \mathfrak{B}^{-}(X)$. Таким образом, мы получаем следующую вспомогательную аппроксимационную лемму.

Лемма 2.5. Пусть $X$ - двусторонний марковский компакт, а его матрииъ смежсности $A_{n}, n \in \mathbb{Z}$, удовлетворяют условию 2.4. Тогда существует такое непрерывное отображение

$$
\Xi^{+}: \operatorname{LipMeas}^{+}(X) \rightarrow \mathfrak{B}^{+}(X),
$$

что для любого $\varepsilon>0$ имеется положительная константа $C_{\varepsilon}$, для которой при любом $x \in X$, любом $n \in \mathbb{N}$ и любой $\Theta \in \operatorname{LipMeas}^{+}(X)$ выполнено

$$
\left|\Theta\left(\gamma_{n}^{+}(x)\right)-\Xi^{+}\left(\Theta ; \gamma_{n}^{+}(x)\right)\right| \leqslant C_{\varepsilon} \cdot\|\Theta\|_{\text {LipMeas }^{+}} \cdot e^{\varepsilon n} .
$$

Отображение $\Xi^{+}$однозначно задается следующим требованием: для любой $\Theta \in \operatorname{LipMeas}^{+}(X)$ и любой $\Phi^{-} \in \mathfrak{B}^{-}(X)$ верно

$$
\left\langle\Theta, \Phi^{-}\right\rangle=\left\langle\Xi^{+}(\Theta), \Phi^{-}\right\rangle
$$




\section{2. Продолжение мер}

2.2.1. Кольцо хорошо приближаемых дуг. Пусть $\mathfrak{R}_{n}^{+}$- кольцо, порожденное полукольцом $\mathfrak{C}_{n}^{+}$. Для подмножества $A$ листа $\gamma_{\infty}^{+}$слоения $\mathscr{F}^{+}$определим множество $\widehat{\gamma}_{n}^{+}(A)$ как минимальный по включению элемент кольца $\mathfrak{R}_{n}^{+}$, содержащий $A$, и, аналогично, определим $\check{\gamma}_{n}^{+}(A)$ как максимальный по включению элемент кольца $\mathfrak{R}_{n}^{+}$, содержащийся в $A$. Разность множеств $\widehat{\gamma}_{n}^{+}(A) \backslash \check{\gamma}_{n}^{+}(A)$ можно единственным образом представить как конечное объединение элементов полукольца $\mathfrak{C}_{n}^{+}$(т. е. дуг вида $\gamma_{n}^{+}(x)$ ). Число таких дуг обозначим через $\delta_{n}^{+}(A)$. Будем говорить, что подмножество $A$ листа $\gamma_{\infty}^{+}$слоения $\mathscr{F}+$ принадлежит семейству $\bar{\Re}^{+}$, если для любого $\varepsilon>0$ существует константа $C_{\varepsilon}$, для которой

$$
\delta_{-n}^{+}(A) \leqslant C_{\varepsilon} e^{\varepsilon n} .
$$

ПРЕДЛОЖениЕ 2.6. Семейство $\overline{\mathfrak{R}}^{+}$является кольцом.

ДокАЗАТЕЛьСТво следует из включения

$$
\widehat{\gamma}_{n}^{+}(A \cup B) \backslash \check{\gamma}_{n}^{+}(A \cup B) \subset\left(\widehat{\gamma}_{n}^{+}(A) \backslash \check{\gamma}_{n}^{+}(A)\right) \cup\left(\widehat{\gamma}_{n}^{+}(B) \backslash \check{\gamma}_{n}^{+}(B)\right)
$$

и аналогичных включений для разности и пересечения множеств.

ПреДЛОЖЕНИЕ 2.7. Пусть марковский компакт X удовлетворяет условию 2.1. Тогда любая мера $\Phi^{+} \in \mathfrak{B}^{+}(X)$ продолжается до конечно аддитивной меры на кольце $\overline{\mathfrak{R}^{+}}$.

Действительно, если $A \in \overline{\mathfrak{R}}^{+}$, то множество $\widehat{\gamma}_{-n}^{+}(A) \backslash \check{\gamma}_{-n}^{+}(A)$ является объединением элементов полукольца $\mathfrak{C}_{-n}^{+}$, число которых растет субэкспоненциально при $n \rightarrow \infty$.

Таким образом, если марковский компакт является регулярным по Ляпунову и $\Phi^{+} \in \mathfrak{B}^{+}$, то величины

$$
\Phi_{\mathbf{v}}^{+}\left(\widehat{\gamma}_{-n-1}^{+}(A) \backslash \widehat{\gamma}_{-n}^{+}(A)\right), \quad \Phi_{\mathbf{v}}^{+}\left(\widehat{\gamma}_{-n}^{+}(A) \backslash \check{\gamma}_{-n}^{+}(A)\right)
$$

убывают экспоненциально быстро при $n \rightarrow \infty$.

Следовательно, мы можем положить

$$
\Phi^{+}(A)=\lim _{n \rightarrow \infty} \Phi^{+}\left(\widehat{\gamma}_{-n}^{+}(A)\right)=\lim _{n \rightarrow \infty} \Phi^{+}\left(\check{\gamma}_{-n}^{+}(A)\right) .
$$

Полученное продолжение конечно аддитивно: если $A, B \in \overline{\mathfrak{R}}^{+}$не пересекаются, то

$$
\check{\gamma}_{n}^{+}(A) \sqcup \check{\gamma}_{n}^{+}(B) \subset \check{\gamma}_{n}^{+}(A \sqcup B), \quad \widehat{\gamma}_{n}^{+}(A \sqcup B) \subset \widehat{\gamma}_{n}^{+}(A) \cup \widehat{\gamma}_{n}^{+}(B) .
$$

Более того, разность между правой и левой частью в каждом из этих включений состоит из конечного числа дуг, которое, по определению кольца $\overline{\mathfrak{R}}^{+}$, растет субэкспоненциально с ростом $n$. Поскольку, по определению, значения $\Phi^{+}$на каждой дуге убывают экспоненциально, величина

$$
\Phi^{+}\left(\widehat{\gamma}_{n}^{+}(A \sqcup B) \backslash \widehat{\gamma}_{n}^{+}(A) \cup \widehat{\gamma}_{n}^{+}(B)\right)
$$


убывает экспоненциально, откуда вытекает конечная аддитивность продолжения (7).

Утверждение предварительной аппроксимационной леммы 2.5 также можно распространить на дуги из кольца $\overline{\mathfrak{R}}^{+}$. Для дуги $\gamma \in \overline{\mathfrak{R}}^{+}$обозначим через $n^{+}(\gamma)$ наибольшее целое $n$, для которого в $\gamma$ содержится непустая дуга из $\mathfrak{C}_{n}^{+}$. Нам также понадобится следующее определение.

ОПРЕДЕЛЕНИЕ. Будем говорить, что последовательность неотрицательных $(m \times m)$-матриц $A_{n}, n \in \mathbb{Z}$, имеет субэкспоненциальный рост, если для любого $\varepsilon>0$ существует такая константа $C_{\varepsilon}$, что при всех $n \in \mathbb{Z}$

$$
\sum_{i=1}^{m} \sum_{j=1}^{m}\left(A_{n}\right)_{i j}<C_{\varepsilon} e^{\varepsilon|n|} .
$$

Теперь мы готовы сформулировать утверждение об аппроксимируемости липшицевых мер на дугах из кольца $\overline{\mathfrak{R}}^{+}$.

СлеДСтвиЕ 2.8. Пусть $X$ - марковский компакт, удовлетворяющий условию 2.4, матрицы смежности которого имеют субэкспоненииальный рост. Пусть $\Xi^{+}$- отображсение, заданное в лемме 2.5. Тогда для каждого $\varepsilon>0$ существует положительная константа $C_{\varepsilon}$ такая, что для любых $\gamma \in \overline{\mathfrak{R}}^{+} u$ $\Theta \in \operatorname{LipMeas}^{+}(X)$ выполнено неравенство

$$
\left|\Theta(\gamma)-\Xi^{+}(\Theta ; \gamma)\right| \leqslant C_{\varepsilon}\|\Theta\|_{\text {LipMeas }}+e^{\varepsilon n^{+}(\gamma)} .
$$

ДокАЗАТЕЛЬСтво. Для дуг из полукольца $\mathfrak{C}_{n}^{+}$это в точности утверждение леммы 2.5. Приближая дугу $\gamma \in \overline{\mathfrak{R}}^{+}$дугами из $\mathfrak{C}_{n}^{+}$и суммируя получающуюся геометрическую прогрессию, получим требуемую оценку.

\subsection{2. Продолжение на дуги при наличии упорядочения Вершика.} Пусть теперь $X$ - марковский компакт с упорядочением Вершика o. Нашей целью является продолжение конечно аддитивной меры из $\mathfrak{B}^{+}$на кольцо, порожденное классом интервалов всех типов: $\left[x, x^{\prime}\right],\left(x, x^{\prime}\right],\left[x, x^{\prime}\right),\left(x, x^{\prime}\right)$ - относительно упорядочивания $\mathfrak{o}$. Пусть $\mathfrak{C}(\mathfrak{o})$ - полукольцо дуг вида $\left[x, x^{\prime}\right],\left(x, x^{\prime}\right]$, $\left[x, x^{\prime}\right),\left(x, x^{\prime}\right)$, а $\mathfrak{R}(\mathfrak{o})$ - кольцо, порожденное этим полукольцом $\mathfrak{C}(\mathfrak{o})$.

Покажем, что достаточным условием для существования продолжения мер из $\mathfrak{B}^{+}$будет субэкспоненциальный рост матриц $A_{n}$.

ПреДЛОЖЕНИЕ 2.9. Пусть $X$ - марковский компакт с упорядочением Вершика о. Если последовательность $A_{n}, n \in \mathbb{Z}$, его матрии, смежности имеет субэкспоненциальный рост, то для любых $x \in X, x^{\prime} \in \gamma_{\infty}^{+}(x)$ интервалы $\left[x, x^{\prime}\right]$, $\left(x, x^{\prime}\right),\left[x, x^{\prime}\right),\left(x, x^{\prime}\right]$ принадлежат кольчу $\bar{\Re}^{+}$.

Доказательство этого предложения основано на рассуждении типа ДанжуаКоксма, которое в нашем случае становится следующим простым наблюдением о разложении дуг $\left[x, x^{\prime}\right],\left(x, x^{\prime}\right),\left[x, x^{\prime}\right),\left(x, x^{\prime}\right]$.

ПреДлОЖеНИЕ 2.10. Для любого $l \in \mathbb{Z}$ существует иисло $N_{l}$, удовлетворяющее неравенству

$$
N_{l} \leqslant C_{\varepsilon} e^{\varepsilon|l|},
$$

для которого верно следующее. 
Пусть $l \in \mathbb{Z}$, и пусть $\gamma-$ дуга из полукольца $\mathfrak{C}(\mathfrak{o})$ такая, ито $\check{\gamma}_{l}(\gamma)=\varnothing$. Тогда

$$
\gamma=\gamma^{\prime} \sqcup\left(\bigsqcup_{k=1}^{N_{l}} \gamma_{l, k}\right) \sqcup \gamma^{\prime \prime},
$$

где $\gamma_{l, k} \in \mathfrak{C}_{l-1}^{+} u \check{\gamma}_{l-1}\left(\gamma^{\prime}\right)=\check{\gamma}_{l-1}\left(\gamma^{\prime \prime}\right)=\varnothing$ (некоторье из дуг могут бъть пустыми).

Другими словами, если дуга из полукольца $\mathfrak{C}(\mathfrak{o})$ не содержит дуг из полукольца $\mathfrak{C}_{l}^{+}$, то она не может содержать более $C_{\varepsilon} e^{\varepsilon|l|}$ дуг из полукольца $\mathfrak{C}_{l-1}^{+}$.

ДокАЗАТЕЛЬСтво. Положим

$$
N_{l}=2 \max _{k} \sum_{i, k=1}^{m}\left(A_{l}\right)_{i k}+1 .
$$

Ясно, что если дуга из полукольца $\mathfrak{C}(\mathfrak{o})$ содержит $N_{l}$ дуг из $\mathfrak{C}_{l-1}^{+}$, то она также содержит и дугу из $\mathfrak{C}_{l}^{+}$. Неравенство (8) вытекает из субэкспоненциальности роста.

Из предложения 2.10 непосредственно следует предложение 2.9 .

\section{3. Гёльдеровская верхняя оценка}

Пусть марковский компакт $X$ строго эргодичен. Обозначим через $\nu^{+} \in$ $\mathfrak{V}^{+}(X)$ положительную меру, а через $h^{(n)}-$ соответствующую эквивариантную последовательность, нормализованные обычным образом. Проверим теперь, что если компоненты векторов $h^{(n)}$ убывают не быстрее чем экспоненциально, то любая конечно аддитивная мера $\Phi^{+} \in \mathfrak{B}^{+}$удовлетворяет неравенству типа Гёльдера по отношению к мере $\nu^{+}$.

УСловие 2.11. Существуют такие константы $C>0, \beta>0$, что вектори $h^{(n)}$ удовлетворяют оценке

$$
\min _{i \in\{1, \ldots, m\}} h_{i}^{(-n)} \geqslant C e^{-\beta n}
$$

ПреДЛОЖЕНИЕ 2.12. Пусть $X$ - строго эргодический марковский компакт, удовлетворяющий условию 2.11. Тогда для любой $\Phi^{+} \in \mathfrak{B}^{+}$существуют такие $\varepsilon>0, \theta>0$, что если $\gamma \in \mathfrak{C}^{+}$такова, ито $\nu^{+}(\gamma) \leqslant \varepsilon$, mо

$$
\left|\Phi^{+}(\gamma)\right| \leqslant\left(\nu^{+}(\gamma)\right)^{\theta}
$$

ДоказАтельство. Действительно, рассмотрим $\gamma \in \mathfrak{C}^{+}, \gamma=\gamma_{-n}^{+}(x)$. Не умаляя общности, можно считать, что $n>0$.

Тогда, по определению пространства $\mathfrak{B}^{+}(X)$, существуют константы $\widetilde{C}>0$, $\alpha>0$, зависящие только от $\Phi^{+}$, такие, что

$$
\left|\Phi^{+}(\gamma)\right| \leqslant \widetilde{C} e^{-\alpha n}
$$


С другой стороны, по условию 2.11 имеем

$$
\nu^{+}(\gamma)=h_{F\left(x_{-n}\right)}^{(-n-1)} \geqslant C e^{-\gamma n} .
$$

Объединяя эти две оценки и выбирая $\varepsilon$ достаточно малым, получаем требуемую оценку с любым $\theta$, для которого $0<\theta<\alpha / \gamma$. Предложение доказано.

Верно следующее частичное обращение предложения 2.12 .

ПреДЛОЖенИЕ 2.13. Пусть строго эргодический марковский компакт $X$ таков, что $\nu^{+} \in \mathfrak{B}^{+}$. Рассмотрим $\Phi^{+} \in \mathfrak{V}^{+}(X)$ и предположим, что существуют $\varepsilon>0, \theta>0$ такие, что если для $\gamma \in \mathfrak{C}^{+}$выполнено неравенство $\nu^{+}(\gamma) \leqslant \varepsilon, m o$

$$
\left|\Phi^{+}(\gamma)\right| \leqslant\left(\nu^{+}(\gamma)\right)^{\theta}
$$

Тогда $\Phi^{+} \in \mathfrak{B}^{+}$.

ДокАЗАТЕЛЬство. Из условия $\nu^{+} \in \mathfrak{B}^{+}$следует, что нормы векторов положительной эквивариантной последовательности $h^{(n)}$ убывают экспоненциально быстро при $n \rightarrow-\infty$, а в силу (9) то же верно и для $\Phi^{+}$, откуда вытекает требуемое.

ЗАмечАниЕ. Те же предложения, очевидно, справедливы и для конечно аддитивных мер на слоениях $\mathscr{F}^{-}$.

ПРЕДЛОЖениЕ 2.14. Пусть для строго эргодичного марковского компакта $X$ верно следующее:

1) $\nu^{+} \in \mathfrak{B}^{+}(X)$;

2) $X$ удовлетворяет условию 2.11 ;

3) последовательность $A_{n}(X), n \in \mathbb{Z}$, имеет субэкспоненииальный рост. Пусть о - упорядочение Вершика на $X$. Тогда для любого $\Phi^{+} \in \mathfrak{B}^{+}(X)$ существуют такие $\theta>0, \varepsilon>0$, что для любых $x \in X, x^{\prime} \in \gamma_{\infty}^{+}(x)$, для которьх

$$
\nu^{+}\left(\left[x, x^{\prime}\right]\right) \leqslant \varepsilon
$$

выполнено неравенство

$$
\Phi^{+}\left(\left[x, x^{\prime}\right]\right) \leqslant \nu^{+}\left(\left[x, x^{\prime}\right]\right)^{\theta} .
$$

Чтобы доказать это предложение, переформулируем сначала предложение 2.10 следующим образом.

Лемма 2.15. Предположим, что для марковского компакта $X$ последовательность $A_{n}(X), n \in \mathbb{Z}$, имеет субэкспоненииальный рост.

Тогда для любого $l \in \mathbb{Z}$ существует число $M_{l}$, удовлетворяющее неравенству

$$
M_{l} \leqslant C_{\varepsilon} e^{\varepsilon|l|}
$$

для которого верно следующее.

Пусть $\gamma$ - дуга из полукольца $\mathfrak{C}(\mathfrak{o})$. Тогда существует разложение

$$
\gamma=\bigsqcup_{l=n^{+}(\gamma)}^{-\infty} \bigsqcup_{k=1}^{M_{l}} \gamma_{l, k},
$$

где $\gamma_{l, k} \in \mathfrak{C}_{l}^{+}$. 


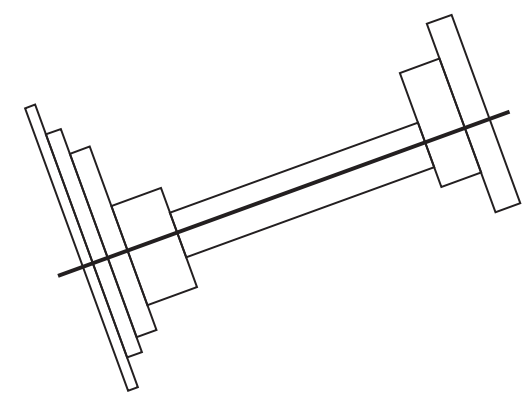

Рис. 1. Число коротких дуг растет не быстрее чем субэкспонециально

Говоря неформально, лемма 2.15 утверждает, что любую дугу нашего символического потока можно приблизить "марковскими" дугами с субэкспоненциальной ошибкой; см. рис. 1. Аналогичные леммы о разложении дуг использовали А. В. Зорич [48], [49] и Дж. Форни [20]-[23].

Предложение 2.14 теперь непосредственно следует из предложения 2.12 и леммы 2.15 .

\section{4. Конкатенация и агрегация}

Конкатенация ГГ $\Gamma^{\prime} \in \mathfrak{G}$ двух графов $\Gamma, \Gamma^{\prime} \in \mathfrak{G}$ определяется следующим образом. Множество ребер $\mathscr{E}\left(\Gamma \Gamma^{\prime}\right)$ задается формулой

$$
\mathscr{E}\left(\Gamma \Gamma^{\prime}\right)=\left\{\left(e, e^{\prime}\right), e \in \mathscr{E}(\Gamma), e \in \mathscr{E}\left(\Gamma^{\prime}\right), I(e)=F\left(e^{\prime}\right)\right\}
$$

и мы полагаем $F\left(e, e^{\prime}\right)=F(e), I\left(e, e^{\prime}\right)=I\left(e^{\prime}\right)$. Ясно, что $A\left(\Gamma \Gamma^{\prime}\right)=A\left(\Gamma^{\prime}\right) A(\Gamma)$.

Пусть $W(\mathfrak{G})$ - множество всех конечных слов в алфавите $\mathfrak{G}$. Для слова $w \in W(\mathfrak{G}), w=w_{0} \ldots w_{n}, w_{i} \in \mathfrak{G}$, будем через $\Gamma(w)$ обозначать конкатенацию $w_{0} \ldots w_{n}$ графов $w_{0}, \ldots, w_{n}$.

Теперь рассмотрим последовательность графов $\Gamma_{n} \in \mathfrak{G}, n \in \mathbb{Z}$, и строго возрастающую последовательность индексов $i_{n} \in \mathbb{Z}, n \in \mathbb{Z}$. Рассмотрим конкатенации

$$
\check{\Gamma}_{n}=\Gamma_{i_{n}} \ldots \Gamma_{i_{n+1}-1} .
$$

Последовательность $\check{\Gamma}_{n}$ будем называть агрегацией последовательности $\Gamma_{n}$, а последовательность $\Gamma_{n}-$ измельчением последовательности $\check{\Gamma}_{n}$.

Пусть $X$ - марковский компакт, отвечающий последовательности $\Gamma_{n}$, а $\check{X}-$ марковский компакт, отвечающий последовательности $\check{\Gamma}_{n}$.

Имеется естественный “тавтологический” гомеоморфизм

$$
\mathfrak{t}_{\mathrm{Ag}\left(i_{n}\right)}: X \rightarrow \check{X}
$$

По определению, гомеоморфизм $\mathfrak{t}_{\mathrm{Ag}\left(i_{n}\right)}$ переводит слоения $\mathscr{F}_{X}^{ \pm}$в соответствующие слоения $\mathscr{F}_{\check{X}}^{ \pm}$и отождествляет пространства $\mathfrak{V}^{ \pm}(X)$ и $\mathfrak{V}^{ \pm}(\check{X})$.

Марковский компакт $X$ строго эргодичен тогда и только тогда, когда строго эргодичен $\check{X}$, и если $\Phi^{+} \in \mathfrak{B}^{+}(X)$, то $\left(\mathfrak{t}_{\mathrm{Ag}\left(i_{n}\right)}\right)_{*} \Phi^{+} \in \mathfrak{B}^{+}(\check{X})$. 


\section{5. Символические потоки}

Пусть $X$ - строго эргодический марковский компакт, соответствующий последовательности графов $\Gamma_{l}$, и пусть для каждого из графов $\Gamma_{l}$ задано упорядочение Вершика. Эти упорядочения индуцируют линейный порядок на каждом листе $\gamma_{\infty}^{+}(x)$.

Пусть $\operatorname{Max}(\mathfrak{o})$ - множество точек $x \in X, x=\left(x_{n}\right)_{n \in \mathbb{Z}}$, таких, что каждое из $x_{n}$ является максимальным ребром. Аналогично, $\operatorname{Min}(\mathfrak{o})-$ множество точек $x \in X, x=\left(x_{n}\right)_{n \in \mathbb{Z}}$, для которых каждое из $x_{n}$ является минимальным ребром. Поскольку ребра, начинающиеся в каждой из вершин, упорядочены линейно, число элементов в каждом из множеств $\operatorname{Max}(\mathfrak{o})$ и $\operatorname{Min}(\mathfrak{o})$ не превосходит $m$.

Если лист $\gamma_{\infty}^{+}$не пересекает $\operatorname{Max}(\mathfrak{o})$, то он не имеет максимального элемента; аналогично, если лист $\gamma_{\infty}^{+}$не пересекает $\operatorname{Min}(\mathfrak{o})$, то он не имеет минимального элемента.

ПредЛОЖение 2.16. Пусть $x \in X . \quad$ Если $\gamma_{\infty}^{+}(x) \cap \operatorname{Max}(\mathfrak{o})=\varnothing$, mо любого $t \geqslant 0$ существует такая точка $x^{\prime} \in \gamma_{\infty}^{+}(x)$, что

$$
\nu_{X}^{+}\left(\left[x, x^{\prime}\right]\right)=t
$$

ДоказАтельство. Пусть $V(x)=\left\{t: \exists x^{\prime} \geqslant x: \nu_{X}^{+}\left(\left[x, x^{\prime}\right]\right)=t\right\}$. Так как $\gamma_{\infty}^{+}(x) \cap \operatorname{Max}(\mathfrak{o})=\varnothing$, для любого $n$ существует $x^{\prime \prime} \in \gamma_{\infty}^{+}(x)$, для которого все точки в $\gamma_{n}^{+}\left(x^{\prime \prime}\right)$ больше, чем $x$. По условию 1.1 величины $\nu_{X}^{+}\left(\gamma_{n}^{+}\left(x^{\prime \prime}\right)\right)$ равномерно по $x^{\prime \prime}$ стремятся к бесконечности при $n \rightarrow \infty$. Следовательно, множество $V(x)$ неограничено. Более того, по условию 1.1 величины $\nu_{X}^{+}\left(\gamma_{n}^{+}\left(x^{\prime \prime}\right)\right)$ стремятся к нулю при $n \rightarrow-\infty$ равномерно по $x^{\prime \prime}$, поэтому множество $V(x)$ плотно в $\mathbb{R}_{+}$. Наконец, из компактности $X$ следует замкнутость множества $V(x)$, что завершает доказательство.

Сходное предложение имеет место для отрицательных $t$. Его доказательство аналогично предыдущему.

ПРЕДЛОЖЕНИЕ 2.17. Пусть $x \in X . E c л u \gamma_{\infty}^{+}(x) \cap \operatorname{Min}(\mathfrak{o})=\varnothing$, mo для любого $t \geqslant 0$ существует точка $x^{\prime} \in \gamma_{\infty}^{+}(x)$, для которой

$$
\nu_{X}^{+}\left(\left[x^{\prime}, x\right]\right)=t
$$

Наша следующая цель - построение потока $h_{t}^{+}$, для которого при всех $t \geqslant 0$ выполнено $h_{t}^{+} x \in \gamma_{\infty}^{+}(x)$ и $\nu_{X}^{+}\left(\left[x, h_{t}^{+} x\right]\right)=t$. Заметим, однако, что указанные условия не определяют точку $h_{t}^{+} x$ однозначно. Поэтому мы модифицируем марковский компакт $X$, склеивая пары точек $x, x^{\prime}$, для которых $x<x^{\prime}$, но $\left(x, x^{\prime}\right)=\varnothing$.

ПреДЛОжЕНИЕ 2.18. Пусть $x, x^{\prime} \in X$ таковъ, что $x \in \gamma_{\infty}^{+}\left(x^{\prime}\right), x<x^{\prime} u$ $\nu_{X}^{+}\left(\left[x, x^{\prime}\right]\right)=0$. Тогда существует $n \in \mathbb{Z}$, для которого

1) $x_{n}^{\prime}$ - это ребро, следующее за $x_{n}$;

2) $x$ - максимальный элемент в $\gamma_{n}(x)$;

3) $x^{\prime}$ - минимальный элемент в $\gamma_{n}\left(x^{\prime}\right)$. 
Определим следующее отношение $\sim_{\mathfrak{o}}$ на $X: x \sim_{\mathfrak{o}} x^{\prime}$, если $x \in \gamma_{\infty}^{+}\left(x^{\prime}\right)$ и $\left(x, x^{\prime}\right)=\left(x^{\prime}, x\right)=\varnothing$. В силу предложения 2.18 оно является отношением эквивалентности, причем его классы содержат не более двух точек, а $\nu$-почти все из них - только одну точку.

Пусть $X_{\mathfrak{o}}=X / \sim_{\mathfrak{o}}, \pi_{\mathfrak{o}}: X \rightarrow X_{\mathfrak{o}}$ - естественная проекция и $\nu_{\mathfrak{o}}=\left(\pi_{\mathfrak{o}}\right)_{*} \nu$. Вероятностные пространства $\left(X_{\mathfrak{o}}, \nu_{\mathfrak{o}}\right)$ и $(X, \nu)$ изоморфны как пространства с мерой; в дальнейшем мы поэтому будем часто опускать индекс $\mathfrak{o}$. Слоения $\mathscr{F}^{+}$и $\mathscr{F}^{-}$ также опускаются на пространство $X_{\mathfrak{o}}$; их образы на $X_{\mathfrak{o}}$ мы будем обозначать теми же буквами и, как и выше, будем обозначать символами $\gamma_{\infty}^{+}(x), \gamma_{\infty}^{-}(x)$ листы слоений, содержащие $x \in X_{\mathfrak{o}}$.

Допустим теперь, что $x \in X_{\mathfrak{o}}$ такова, что $\gamma_{\infty}^{+}(x) \cap \operatorname{Max}(\mathfrak{o})=\varnothing . \quad$ В силу предложения 2.16 для любого $t \geqslant 0$ существует единственная точка $x^{\prime}$, удовлетворяющая (10). Обозначим $h_{t}^{+}(x)=x^{\prime}$. Аналогично, если $x \in X_{\mathfrak{o}}$ такова, что $\gamma_{\infty}^{+}(x) \cap \operatorname{Min}(\mathfrak{o})=\varnothing$, то, по предложению 2.17 , для любого $t \geqslant 0$ существует единственная $x^{\prime}$, для которой выполнено (11). Обозначим $h_{-t}^{+}(x)=x^{\prime}$.

Таким образом, мы получили поток $h_{t}^{+}$, который корректно определен на множестве

$$
X_{\mathfrak{o}} \backslash\left(\bigcup_{x \in \operatorname{Max}(\mathfrak{o}) \cup \operatorname{Min}(\mathfrak{o})} \gamma_{\infty}^{+}(x)\right) .
$$

В частности, он определен $\nu$-почти всюду на $X_{\mathfrak{o}}$. По определению, поток $h_{t}^{+}$ сохраняет меру $\nu$.

Поток $h_{t}^{+}$является специальным потоком над автоморфизмом Вершика, отвечающим одностороннему марковскому компакту $Y$, заданному последовательностью графов $\Gamma_{n}, n \geqslant 1$. Специальная функция для этого потока является кусочно постоянной функцией $h_{F\left(y_{1}\right)}^{(1)}$.

Упорядочение Вершика на графах $\Gamma, \Gamma^{\prime}$ задает упорядочение на их конкатенации ГГ': мы полагаем $\left(e, e^{\prime}\right)<\left(\widetilde{e}, \widetilde{e}^{\prime}\right)$, если $e^{\prime}<\widetilde{e}^{\prime}$ или если $e^{\prime}=\widetilde{e}^{\prime}, e<\widetilde{e}$.

Таким образом, если марковский компакт $X$ снабжен упорядочением Вершика $\mathfrak{o}$, а марковский компакт $\check{X}$ получается из $X$ агрегацией графов, заданной строго возрастающей последовательностью индексов $\left(i_{n}\right)$, то $\check{X}$ также автоматически снабжается упорядочением Вершика о̆ и отображение $\mathfrak{t}_{\mathrm{Ag}\left(i_{n}\right)}$ переводит поток $h_{t}^{+, \mathfrak{o}}$ на $X$ в поток $h_{t}^{+, \check{\mathfrak{o}}}$ на $\check{X}$.

Аналогичным образом, предположим, что для любого графа $\Gamma_{n}, n \in \mathbb{Z}$, задан линейный порядок $\widetilde{\mathfrak{o}}$ на множестве ребер, заканчивающихся в данной вершине. Такой набор порядков будем называть обратным упорядочением Вершикa. Аналогично предыдущему, обратное упорядочение Вершика задает поток на листах слоения $\mathscr{F}^{-}$, сохраняющий меру $\nu$.

\section{6. Гёльдеровские коциклы}

Как и раньше, мы рассматриваем строго эргодический марковский компакт $X$ с упорядочением Вершика о и с порожденным им потоком $h_{t}^{+}$. Под дугой потока $h_{t}^{+}$мы будем понимать множество вида

$$
\gamma(x, t)=\left\{y \in \gamma^{+}(x), x \leqslant y<h_{t}^{+}(x)\right\}, \quad x \in X, \quad t \geqslant 0 .
$$


Другими словами, дуга - это образ интервала $\left[x, x^{\prime}\right)=\left\{x^{\prime \prime}: x \leqslant x^{\prime \prime}<x^{\prime}\right\}$ под действием факторизации по отношению эквивалентности $\sim_{\mathfrak{o}}$.

В силу предложения 2.9 если матрицы смежности марковского компакта имеют субэкспоненциальный рост, то все конечно аддитивные меры из $\mathfrak{B}^{+}$ можно продолжить на произвольные дуги потока $h_{t}^{+}$. Иными словами, верно следующее предложение.

Предложение 2.19. Любая дуга потока $h_{t}^{+}$принадлежит кольиу $\overline{\mathfrak{R}}^{+}$.

Поскольку любая мера $\Phi^{+} \in \mathfrak{B}^{+}$определена на любой дуге потока $h_{t}^{+}$, такая мера задает коцикл на орбитах потока $h_{t}^{+}$по формуле

$$
\Phi^{+}(x, t)=\Phi^{+}\left(\left[x, h_{t}^{+} x\right]\right) .
$$

Несколько неформально в дальнейшем мы будем отождествлять меру и соответствующий ей коцикл и обозначать их одной и той же буквой, говоря, например, о норме коцикла вместо нормы соответствующей конечно аддитивной меры и т. п.

\section{7. Сбалансированные, регулярные по Ляпунову и гиперболические марковские компакты}

Более точные утверждения о гёльдеровском поведении коциклов можно получить при более сильных предположениях на матрицы смежности марковского компакта. Предположения, которые мы сформулируем, наверняка могут быть ослаблены; тем не менее они выполняются для случайных марковских компактов и потому достаточны для наших целей. Перейдем к формальным определениям.

Марковский компакт называют сбалансированным, если выполнено следующее условие.

Условие 2.20. Существуют положительная константа С и такая строго возрастающая последовательность индексов $i_{n} \in \mathbb{Z}, n \in \mathbb{Z}$, что

1) матрицы $A_{i_{n+1}} \cdots A_{i_{n}+1}$ имеют субэкспоненциалъный рост по $n \in \mathbb{Z}$;

2) для любого $n \in \mathbb{Z}$ все элементы матрицы $A_{i_{n+1}} \cdots A_{i_{n}+1}$ положительны, и для любых $j, k, l \in\{1, \ldots, m\}$ верно неравенство

$$
\frac{\left(A_{i_{n+1}} \cdots A_{i_{n}+1}\right)_{j k}}{\left(A_{i_{n+1}} \cdots A_{i_{n}+1}\right)_{l k}} \leqslant C .
$$

Достаточным условием для выполнения второго требования является следующее: существуют матрица $Q$, все элементы которой положительны, и последовательность индексов $i_{n}$, для которых $A_{i_{n}}=Q$. Мы увидим, что марковские компакты, случайные относительно некоторой $\sigma$-инвариантной эргодической вероятностной меры $\mu$ на $\Omega$ и удовлетворяющие условию 1.2 , автоматически оказываются сбалансированными.

Отметим также, что сбалансированные марковские компакты являются строго эргодическими. 
Сбалансированный марковский компакт, удовлетворяющий условию 2.1, называется регулярным по Ляпунову, если дополнительно выполняется следующее условие.

УСловие 2.21. 1. В условии 2.20 константу $C$ можно выбрать таким образом, что последовательность $i_{n}$ имеет положительную плотность, т.е. для некоторых $n_{0}, \alpha>0$ верно, что $\left|i_{n}\right|<\alpha|n|$ при всех $n,|n|>n_{0}$.

2. Существуют такие положительные числа $\theta_{1}>\theta_{2}>\cdots>\theta_{l_{0}}>0, a$ также, при всех $n \in \mathbb{Z}$, такое разложение в прямую сумму

$$
E_{n}^{u}=E_{n}^{1} \oplus E_{n}^{2} \oplus \cdots \oplus E_{n}^{l_{0}}
$$

что $A_{n} E_{n}^{i}=E_{n+1}^{i}$ иля любого ненулевого $v \in E_{n}^{i}$ имеют место соотношения

$$
\begin{aligned}
\lim _{k \rightarrow \infty} \frac{\log \left|A_{n-k} \cdots A_{n} v\right|}{k} & =\theta_{i}, \\
\lim _{k \rightarrow \infty} \frac{\log \left|\left(A_{n-k} \cdots A_{n}\right)^{-1} v\right|}{k} & =-\theta_{i} .
\end{aligned}
$$

Сходимость в (12), (13) равномерная по единичной сфере $\left\{v \in E_{n}^{i},|v|=1\right\}$.

Отметим, что положительная эквивариантная последовательность $h^{(n)}$ сбалансированного регулярного по Ляпунову марковского компакта автоматически удовлетворяет условию 2.11.

Пусть теперь $X$ строго эргодичен. Зададимся $v \in E_{0}^{u}$ и рассмотрим соответствующую конечно аддитивную меру $\Phi^{+} \in \mathfrak{B}^{+}$. Разложим $v=v^{(1)}+\cdots+v^{\left(l_{0}\right)}$, $v^{(i)} \in E_{0}^{i}$, и положим $j$ равным наименьшему индексу, для которого $v^{(j)} \neq 0$. Тогда $\theta_{j}$ называется показателем Ляпунова конечно аддитивной меры $\Phi^{+}$. Например, положительная мера имеет показатель $\theta_{1}$. Аналогично, пусть $k-$ это наибольший индекс, для которого $v^{(k)} \neq 0$. Тогда $\theta_{k}$ называется наименъшим показателем Ляпунова меры $\Phi^{+}$. Оказывается, что показатель Ляпунова меры $\Phi^{+}$отвечает за ее рост на бесконечности, а наименьший показатель Ляпунова - за ее гёльдеровское поведение в нуле.

Нам часто будет нужно условие, двойственное к условию 2.21. Рассмотрим марковский компакт $X$, удовлетворяющий условиям 2.1, 2.4, 2.21. Определим

$$
\widetilde{E}_{n}^{i}=\operatorname{Ann}\left(\bigoplus_{j \neq i} E_{n}^{j}\right) \quad \text { при } i=1, \ldots, l_{0} .
$$

Тогда

$$
\widetilde{E}_{n}^{u}=\widetilde{E}_{n}^{1} \oplus \widetilde{E}_{n}^{2} \oplus \cdots \oplus \widetilde{E}_{n}^{l_{0}}
$$

и $A_{n}^{t} \widetilde{E}_{n+1}^{i}=\widetilde{E}_{n}^{i}$. 
УСловиЕ 2.22. Для любого $n \in \mathbb{Z}$ и любого ненулевого $v \in \widetilde{E}_{n}^{i}$ имеют место соотношения

$$
\begin{aligned}
\lim _{k \rightarrow \infty} \frac{\log \left|A_{n-k}^{t} \cdots A_{n}^{t} v\right|}{k} & =\theta_{i}, \\
\lim _{k \rightarrow \infty} \frac{\log \left|\left(A_{n+k}^{t} \cdots A_{n}^{t}\right)^{-1} v\right|}{k} & =-\theta_{i} .
\end{aligned}
$$

Сходимость в (14), (15) равномерная на единичной сфере $\left\{v \in \widetilde{E}_{n}^{i},|v|=1\right\}$.

Марковский компакт, удовлетворяющий условиям 2.21, 2.22, будем называть бирегулярным по Ляпунову.

ЗАмечаниЕ. Равномерность сходимости в (14), (15) гарантируется мультипликативной эргодической теоремой Оселедца (см. [5] и приложение А).

Особый интерес для нас представляет случай, когда все показатели Ляпунова являются простыми, т. е. когда выполнено следующее условие.

УСловие 2.23. Верно, что $l_{0}=\operatorname{dim} E_{n}^{u}=\operatorname{dim} \widetilde{E}_{n}^{u} u$

$$
\operatorname{dim} E_{n}^{i}=\operatorname{dim} \widetilde{E}_{n}^{i}=1, \quad i=1, \ldots, l_{0} .
$$

При выполнении условия 2.23 будем говорить, что марковский компакт $X$ имеет простой ляпуновский спектр.

Наконец, регулярный по Ляпунову марковский компакт $X$ называется гиперболическим, если $\mathfrak{B}_{c}^{+}(X)=\mathfrak{B}^{+}(X)$.

\section{8. Свойство Гёльдера для коциклов}

2.8.1. Верхняя оценка. Предложение 2.14 можно теперь переформулировать следующим образом.

СлЕДСТВИЕ 2.24. Пусть $X$ - марковский компакт, наибольший показатель Ляпунова которого равен $\theta_{1}$. Тогда для любого $\varepsilon>0$ существует такая положительная константа $C_{\varepsilon}$, зависящая только от компакта $X$, что верно следующее. Пусть $\Phi^{+} \in \mathfrak{B}^{+}$имеет показатель Ляпунова $\theta>0$. Тогда для любого $x \in X$ и любого $t \in \mathbb{R}$ выполнено неравенство

$$
\left|\Phi^{+}(x, t)\right| \leqslant C_{\varepsilon}\left|\Phi^{+}\right||t|^{\theta / \theta_{1}-\varepsilon} .
$$

ЗАмЕчАНИЕ. Поскольку пространство $\mathfrak{B}^{+}$конечномерно, выбор нормы на нем, стоящей в правой части $(16)$, влияет лишь на значение константы $C_{\varepsilon}$.

2.8.2. Логарифмическая асимптотика на бесконечности. Верхняя оценка из следствия 2.24 является точной, как показывает следующее предложение. 
ПреДЛОЖение 2.25. Рассмотрим регулярный по Ляпунову сбалансированный марковский компакт $X$, наибольший показатель Ляпунова которого равен $\theta_{1}$. Пусть $\Phi^{+} \in \mathfrak{B}^{+}$имеет показатель Ляпунова $\theta>0$. Тогда при всех $x \in X$ имеем

$$
\limsup _{t \rightarrow \infty} \frac{\log \left|\Phi^{+}(x, t)\right|}{\log t}=\frac{\theta}{\theta_{1}} .
$$

ДокАЗАтельство. Верхняя оценка получена в следствии 2.24, и нам остается доказать нижнюю. Пусть $v^{(n)}$ - эквивариантная последовательность, отвечающая $\Phi^{+}$. Зафиксируем $\varepsilon>0$. Существует такое $n_{0}$, что при любом $n>n_{0}$ найдется $i \in\{1, \ldots, m\}$, для которого $\left|v_{i}^{(n)}\right| \geqslant e^{(\theta-\varepsilon) n}$.

Теперь рассмотрим $x \in X$ и положим $x^{\prime} \in \gamma_{\infty}^{+}(x)$ равным наименьшему элементу $\gamma_{\infty}^{+}(x)$, удовлетворяющему следующим условиям:

1) $F\left(x_{n+1}^{\prime}\right)=i$;

2) существует $\widetilde{x} \in\left(x, x^{\prime}\right)$, для которого $F\left(\widetilde{x}_{n+1}\right) \neq i$.

Пусть $x^{\prime \prime}>x^{\prime}$ - это наименьший элемент $\gamma_{\infty}^{+}\left(x^{\prime}\right)=\gamma_{\infty}^{+}(x)$, для которого $F\left(x_{n+1}^{\prime \prime}\right) \neq i$.

По определению,

$$
\gamma_{n+1}\left(x^{\prime}\right)=\left[x^{\prime}, x^{\prime \prime}\right) .
$$

Далее, в силу регулярности по Ляпунову марковского компакта $X$

$$
\nu^{+}\left(\left[x, x^{\prime}\right]\right) \leqslant e^{\left(\theta_{1}+\varepsilon\right) n}, \quad \nu^{+}\left(\left[x^{\prime}, x^{\prime \prime}\right]\right) \leqslant e^{\left(\theta_{1}+\varepsilon\right) n} .
$$

Кроме того, верно, что

$$
\left|\Phi^{+}\left(\left[x^{\prime}, x^{\prime \prime}\right]\right)\right|=\left|v_{i}^{(n)}\right| \geqslant e^{(\theta-\varepsilon) n} .
$$

Следовательно, $\max \left(\left|\Phi^{+}\left(\left[x, x^{\prime}\right]\right)\right|,\left|\Phi^{+}\left(\left[x, x^{\prime \prime}\right]\right)\right|\right) \geqslant e^{(\theta-\varepsilon) n} / 2$, а потому верно либо неравенство

$$
\left|\Phi^{+}\left(\left[x, x^{\prime}\right]\right)\right| \geqslant \frac{1}{2}\left(\nu^{+}\left(\left[x, x^{\prime}\right]\right)\right)^{(\theta-\varepsilon) /\left(\theta_{1}+\varepsilon\right)},
$$

либо неравенство

$$
\left|\Phi^{+}\left(\left[x, x^{\prime \prime}\right]\right)\right| \geqslant \frac{1}{2}\left(\nu^{+}\left(\left[x, x^{\prime \prime}\right]\right)\right)^{(\theta-\varepsilon) /\left(\theta_{1}+\varepsilon\right)} .
$$

Таким образом, требуемая нижняя оценка установлена. Рис. 2 иллюстрирует доказательство.

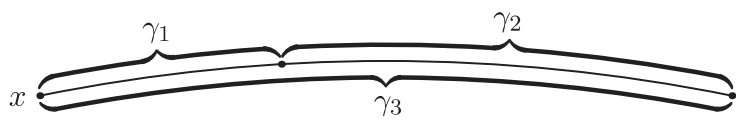

Рис. 2. Доказательство нижней оценки в предложении 2.25: $\gamma_{1}=$ $\left[x, x^{\prime}\right], \gamma_{2}=\left[x^{\prime}, x^{\prime \prime}\right]$. Либо $\gamma_{1}$, либо $\gamma_{3}$ удовлетворяет нижней оценке.

То же рассуждение позволяет доказать, что поведение в нуле в точности гёльдеровское. 
ПреДЛОЖенИЕ 2.26. Пусть $X$ - регулярный по Ляпунову сбалансированный марковский компакт, наибольший показатель Ляпунова которого равен $\theta_{1}$, и пусть $\Phi^{+} \in \mathfrak{B}^{+}$имеет наименьиий показатель Ляпунова $\theta>0$. Тогда для любого $x \in X$

$$
\limsup _{t \rightarrow 0} \frac{\log \left|\Phi^{+}(x, t)\right|}{\log t}=\frac{\theta}{\theta_{1}} .
$$

ДокАзАтельство. Верхняя оценка вытекает из следствия 2.24. Нижняя оценка устанавливается так же, как в предложении 2.25 (с тем изменением, что теперь $n \rightarrow-\infty$, а не $n \rightarrow \infty)$ : сначала находится марковская дуга $\left[x^{\prime}, x^{\prime \prime}\right]$, удовлетворяющая нижней оценке, а затем из регулярности по Ляпунову выводится, что одна из дуг $\left[x, x^{\prime}\right],\left[x, x^{\prime \prime}\right]$ также удовлетворяет требуемой нижней оценке.

\subsection{3. Математическое ожидание и дисперсия для гёльдеровских} коциклов.

ПреДЛОЖениЕ 2.27. Для любого $\Phi^{+} \in \mathfrak{B}^{+}$и любого $t_{0} \in \mathbb{R}$ выполнено равенство

$$
\mathrm{E}_{\nu} \Phi^{+}\left(x, t_{0}\right)=\left\langle\Phi^{+}, \nu^{-}\right\rangle t_{0}
$$

ДокАЗАТЕЛЬство. Поскольку предложение, очевидно, выполняется для $\Phi^{+}=\nu^{+}$, его достаточно доказать в случае $\left\langle\Phi^{+}, \nu^{-}\right\rangle=0$. Если при этом $\mathrm{E}_{\nu} \Phi^{+}(x, t) \neq 0$, то в силу эргодической теоремы

$$
\limsup _{T \rightarrow \infty} \frac{\log \left|\Phi^{+}(x, T)\right|}{\log T}=1,
$$

и потому $\left\langle\Phi^{+}, \nu^{-}\right\rangle \neq 0$.

ПРЕДЛОЖЕНИЕ 2.28. Для любого $\Phi^{+} \in \mathfrak{B}^{+}$, не пропориионалъного $\nu^{+}, u$ любого $t_{0} \neq 0$ имеем

$$
\operatorname{Var}_{\nu} \Phi^{+}\left(x, t_{0}\right) \neq 0 .
$$

ДоказАтельство. Перейдя от $\Phi^{+}{ }_{\mathrm{K}} \Phi^{+}-\left\langle\Phi^{+}, \nu^{-}\right\rangle \nu^{+}$, можно считать, что $\mathrm{E}_{\nu} \Phi^{+}\left(x, t_{0}\right)=0$. Если $\operatorname{Var}_{\nu} \Phi^{+}\left(x, t_{0}\right)=0$, то $\Phi^{+}\left(x, t_{0}\right)$ тождественно равно нулю, но тогда

$$
\limsup _{T \rightarrow \infty} \frac{\log \left|\Phi^{+}(x, T)\right|}{\log T}=0,
$$

откуда $\Phi^{+}=0$, и предложение доказано.

ЗАмечАниЕ. В случае подстановочных динамических систем сходные коциклы рассматривались в работе ЖК.-М. Дюмона, Т. Камае, С. Такахаси [16] и в работе Т. Камае [27].

\section{9. Приближение слабо липшицевых функций}

Из слабой липшицевости функции $f$ вытекают равномерные оценки разности ее интегралов вдоль дуг потока $h_{t}^{+}$, которые остаются внутри некоторого фиксированного марковского прямоугольника (см. рис. 3 ). 


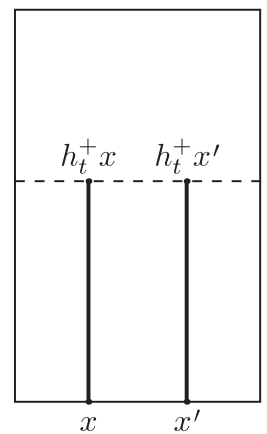

Рис. 3. Дуги траекторий потока остаются в том же марковском прямоугольнике

ПРЕДЛОЖЕНИЕ 2.29. Существует такая константа $C>0$, что для любой функции $f \in \operatorname{Lip}_{w}^{+}(X)$, любого $T>0$ и любой пары точек $x, x^{\prime}$ верно следующее: если существуют такие $i \in\{1, \ldots, m\}$ u $n \in \mathbb{N}$, что для всех $t$, $0 \leqslant t \leqslant T$, верно $F\left(\left(h_{t}^{+} x\right)_{n}\right)=F\left(\left(h_{t}^{+} x^{\prime}\right)_{n}\right)=i$, mо

$$
\left|\int_{0}^{T} f \circ h_{t}^{+}(x) d t-\int_{0}^{T} f \circ h_{t}^{+}\left(x^{\prime}\right) d t\right| \leqslant C\|f\|_{\operatorname{Lip}_{w}^{+}} .
$$

Теперь мы готовы к доказательству основного результата этого раздела.

Лемма 2.30. Пусть $X$ - строго эргодический бирегулярный по Ляпунову сбалансированный марковский компакт. Тогда существует непрерывное отображение $\Xi^{+}: \operatorname{Lip}_{w}^{+}(X) \rightarrow \mathfrak{B}^{+}(X)$, для которого выполнено следующее: для любого $\varepsilon>0$ существует такая константа $C_{\varepsilon}$, ито при всех $f \in \operatorname{Lip}_{w}^{+}(X)$, всех $x \in X$ и всех $T>0$ верно неравенство

$$
\left|\int_{0}^{T} f \circ h_{t}^{+}(x) d t-\Xi^{+}(f ; x, T)\right| \leqslant C_{\varepsilon}\|f\|_{\operatorname{Lip}_{w}^{+}}\left(1+T^{\varepsilon}\right) .
$$

Такое отображение $\Xi^{+}$единственно, если потребовать, что для любого $\Phi^{-} \in$ $\mathfrak{B}^{-}(X)$ выполнено равенство

$$
\left\langle\Xi^{+}(f), \Phi^{-}\right\rangle=\int_{X} f d \nu^{+} \times \Phi^{-} .
$$

ЗАмечАниЕ. Здесь мы пишем $\Xi^{+}(f ; x, T)$ вместо $\Xi^{+}(f)(x, T)$.

ДоказАтельство. Если $f \in \operatorname{Lip}_{w}^{+}(X)$, то мера $f d \nu^{+}$слабо липшицева на слоении $\mathscr{F}^{+}$. Пусть $\Xi^{+}-$непрерывное отображение, даваемое вспомогательной аппроксимационной леммой 2.5. Допуская вольность в обозначениях, положим $\Xi^{+}(f)=\Xi^{+}\left(f d \nu^{+}\right)$. Оценка (17) для "марковских" дуг вида $\gamma_{n}^{+}(x)$ заключена в лемме 2.5, для произвольных же дуг потока $h_{t}^{+}$утверждение вытекает из оценки, приведенной в следствии 2.8, применимость которого к дугам потока следует из предложения 2.9 . 


\subsection{0. Аппроксимационная теорема для случайных марковских компактов}

\subsection{1. Косые произведения, сопоставленные сдвигу и ренормали-} зационному коциклу. Перейдем к доказательству аппроксимационной теоремы для случайных марковских компактов и изучению действий, которые левый сдвиг $\sigma$ на пространстве марковских компактов $\Omega$ индуцирует на пространствах $\mathfrak{B}^{+}$гёльдеровских коциклов на индивидуальных марковских компактах. Это действие будет играть ведущую роль в доказательстве предельных теорем.

Для $\omega \in \Omega$ рассмотрим естественное отображение $\mathfrak{t}_{\sigma}: X(\omega) \rightarrow X(\sigma \omega)$, которое ставит в соответствие точке $x \in X$ точку $\widetilde{x} \in X(\sigma \omega)$, для которой $\widetilde{x}_{n}=x_{n+1}$.

Отображение $\mathfrak{t}_{\sigma}$ переводит слоения $\mathscr{F}_{\omega}^{+}, \mathscr{F}_{\omega}^{-}$в $\mathscr{F}_{\sigma \omega}^{+}, \mathscr{F}_{\sigma \omega}^{-}$, полукольца $\mathfrak{C}_{\omega}^{+}, \mathfrak{C}_{\omega}^{-}$ в $\mathfrak{C}_{\sigma \omega}^{+}, \mathfrak{C}_{\sigma \omega}^{-}$и индуцирует изоморфизм $\left(\mathfrak{t}_{\sigma}\right)_{*}: \mathfrak{V}^{+}\left(X_{\omega}\right) \rightarrow \mathfrak{V}^{+}\left(X_{\sigma \omega}\right)$, задаваемый обычной формулой

$$
\left(\mathfrak{t}_{\sigma}\right)_{*} \Phi^{+}(\gamma)=\Phi^{+}\left(\left(\mathfrak{t}_{\sigma}\right)^{-1} \gamma\right), \quad \gamma \in \mathfrak{C}_{\sigma \omega}^{+}
$$

Определим пространство

$$
\mathfrak{X} \Omega=\{(\omega, x), x \in X(\omega)\}
$$

и зададим на нем отображение $\sigma^{\mathfrak{X}}$, являющееся косым произведением, по формуле

$$
\sigma^{\mathfrak{X}}(\omega, x)=\left(\sigma \omega, \mathfrak{t}_{\sigma} x\right) .
$$

Далее, ренормализационному коциклу $\mathbb{A}$ сопоставим соответствующее отображение косого произведения $\sigma^{\mathbb{A}}: \Omega \times \mathbb{R}^{m} \rightarrow \Omega \times \mathbb{R}^{m}$, заданное формулой

$$
\sigma^{\mathbb{A}}(\omega, v)=(\sigma \omega, \mathbb{A}(1, \omega) v)
$$

Рассмотрим пространство

$$
\mathfrak{V}^{+} \Omega=\left\{\left(\omega, \Phi^{+}\right): \omega \in \Omega_{\text {inv }}, \Phi^{+} \in \mathfrak{V}^{+}(X(\omega))\right\}
$$

и его автоморфизм $\mathfrak{T}_{\sigma}$, задаваемый формулой

$$
\mathfrak{T}_{\sigma}\left(\omega, \Phi^{+}\right)=\left(\sigma \omega,\left(\mathfrak{t}_{\sigma}\right)_{*} \Phi^{+}\right) .
$$

Пусть $\omega \in \Omega_{\text {inv }}, v \in \mathbb{R}^{m}$. Рассмотрим эквивариантую последовательность $\mathbf{v}=\left(v^{(n)}\right)$ такую, что $v^{(0)}=v$. Пусть $\Phi_{\mathbf{v}}^{+} \in \mathfrak{V}^{+}(X(\omega))-$ соответствующая конечно аддитивная мера. Поскольку $v^{(n)}$ определяются по $v^{(0)}$ однозначно, получаем изоморфизм

$$
\mathscr{I}_{\omega}^{+}: \mathbb{R}^{m} \rightarrow \mathfrak{V}^{+}(X(\omega)),
$$

задаваемый как $\mathscr{I}_{\omega}^{+} v=\Phi_{\mathbf{v}}^{+}$, где $\mathbf{v}=\left(v^{(n)}\right)$ - эквивариантная последовательность с $v^{(0)}=v$. 
Ясно, что для любого $\omega \in \Omega_{\text {inv }}$ диаграмма

$$
\begin{array}{cc}
\mathbb{R}^{m} \stackrel{\mathscr{I}_{\omega}^{+}}{\longrightarrow} \mathfrak{V}^{+}(X(\omega)) \\
\downarrow \mathbb{A}(1, \omega) & \downarrow\left(\mathfrak{t}_{\sigma}\right)_{*} \\
\mathbb{R}^{m} \stackrel{\mathscr{I}_{\sigma \omega}^{+}}{\longrightarrow} \mathfrak{V}^{+}(X(\sigma \omega))
\end{array}
$$

коммутативна.

\subsection{2. Свойства случайных марковских компактов.}

ПРЕДЛОЖЕНИЕ 2.31. Если мера $\mu$ удовлетворяет условию 1.2, то для почти всех $\omega$ марковский компакт $X(\omega)$ строго эргодичен и бирегулярен по Ляпунову.

Действительно, строгая эргодичность вытекает непосредственно из первого пункта условия 1.2 , а бирегулярность следует из второго и третьего пунктов по теореме Оселедца.

Для $\omega \in \Omega$ рассмотрим $E_{\omega}^{u}-$ ляпуновское подпространство в точке $\omega$, отвечающее положительным показателям Ляпунова коцикла $\mathbb{A}$. Первый пункт условия 1.2 влечет нетривиальность подпространства $E_{\omega}^{u}$ : действительно, $h_{\omega}^{(0)} \in E_{\omega}^{u}$.

ПРЕДЛОЖенИЕ 2.32. Для $\mu$-почти всех $\omega \in \Omega$ преобразование $\mathscr{I}_{\omega}^{+}$изоморфно отображсает подпространство $E_{\omega}^{u}$ на $\mathfrak{B}^{+}(X(\omega))$.

Это предложение непосредственно следует из мультипликативной теоремы Оселедца.

Для транспонированного коцикла мы аналогично получаем для любых $\omega \in \Omega_{\mathrm{inv}}, v \in \mathbb{R}^{m}$, изоморфизм

$$
\mathscr{I}_{\omega}^{-}: \mathbb{R}^{m} \rightarrow \mathfrak{V}^{-}(X(\omega)),
$$

задаваемый формулой $\mathscr{I}_{\omega}^{-} \widetilde{v}=\Phi_{\widetilde{\mathbf{v}}}^{+}$, где $\widetilde{\mathbf{v}}=\left(\widetilde{v}^{(n)}\right)$ - обратно эквивариантная последовательность, удовлетворяющая условию $\widetilde{v}^{(0)}=\widetilde{v}$.

Как и выше, для любого $\omega \in \Omega_{\text {inv }}$ диаграмма

$$
\begin{aligned}
& \mathbb{R}^{m} \stackrel{\mathscr{I}_{\omega}^{-}}{\longrightarrow} \mathfrak{V}^{-}(X(\omega)) \\
& \uparrow \mathbb{A}^{t}(1, \sigma \omega) \\
& \mathbb{R}^{m} \stackrel{\mathscr{I}_{\sigma \omega}^{-}}{\longrightarrow} \mathfrak{V}^{-}(X(\omega))
\end{aligned}
$$

коммутативна.

Будем далее снова предполагать, что $\mu$ удовлетворяет условию 1.2 , и пусть $\widetilde{E}_{\omega}^{u}$ обозначает ляпуновское подпространство в точке $\omega \in \Omega$, отвечающее положительным показателям Ляпунова для коцикла $\mathbb{A}^{t}$. В силу определения пространств $\mathfrak{B}^{-}(X(\omega))$ и теоремы Оселедца верно следующее предложение.

ПредЛОжение 2.33. Для $\mu$-почти всех $\omega \in \Omega$ преобразование $\mathscr{I}_{\omega}^{-}$изоморфно отображает подпространство $\widetilde{E}_{\omega}^{u}$ на $\mathfrak{B}^{-}(X(\omega))$. 
2.10.3. Двойственность. Рассмотрим $\omega \in \Omega_{\text {inv }}$. Очевидно, что

$$
\left\langle\mathscr{I}_{\omega}^{+}(v), \mathscr{I}_{\omega}^{-}(\widetilde{v})\right\rangle=\sum_{i=1}^{m} v_{i} \widetilde{v}_{i} \quad \text { для } v, \widetilde{v} \in \mathbb{R}^{m} .
$$

Если $\mu$ удовлетворяет условию 1.2, то по теореме Оселедца при почти всех $\omega \in \Omega$ для марковского компакта $X(\omega)$ справедливо условие 2.4. В частности, стандартное евклидово скалярное произведение задает невырожденное спаривание подпространств $E_{\omega}^{u}$ и $\widetilde{E}_{\omega}^{u}$. Таким образом, мы получаем следующее утверждение.

СлЕДСТвИЕ 2.34. Если вероятностная б-инвариантная эргодическая мера н удовлетворяет условию 1.2, то для $\mu$-почти всех $\omega \in \Omega$ спаривание $\langle\cdot, \cdot\rangle$ является невырожденным на паре подпространств $\mathfrak{B}^{+}(X(\omega)), \mathfrak{B}^{-}(X(\omega))$.

\subsection{4. Сбалансированные случайные марковские компакты и ап- проксимационная теорема.}

ПРЕДЛОЖЕНИЕ 2.35. Если для меры $\mu$ выполнено условие 1.2, то для почти всех $\omega$ марковский компакт $X(\omega)$ является сбалансированным.

ДокАЗАТЕЛЬСтво. Проверим, что выполняются все требования условия 2.20. Обозначим $\mathfrak{G}_{+}$множество всех таких $\Gamma \in \mathfrak{G}$, что все элементы матрицы $A(\Gamma)$ положительны, и пусть появление графа $\Gamma_{0} \in \mathfrak{G}_{+}$имеет положительную вероятность относительно $\mu$ (такой $\Gamma_{0}$ существует по первому пункту условия 1.2). Пусть $i_{n}, n \in \mathbb{Z},-$ последовательные моменты времени, когда $\omega_{i_{n}}=\Gamma_{0}$ (последовательность $i_{n}$ почти наверное не ограничена как в положительном, так и в отрицательном направлении). Из положительности $A\left(\Gamma_{0}\right)$ сразу следует второе требование в условии 2.20, и нам остается проверить первое. Обозначим через $\sigma_{\Gamma_{0}}$ индуцированное отображение $\sigma$ на множестве $\left\{\omega: \omega_{0}=\Gamma_{0}\right\}$. Ренормализационный коцикл $\mathbb{A}$ естественным образом задает индуцированный коцикл $\mathbb{A}_{\Gamma_{0}}$ над $\sigma_{\Gamma_{0}}$ (чтобы получить матрицу $\mathbb{A}_{\Gamma_{0}}$, нужно перемножить все матрицы коцикла $\mathbb{A}$ между последовательными появлениями $\left.\Gamma_{0}\right)$. Если логарифм нормы $\mathbb{A}$ интегрируем, то это же верно и для $\mathbb{A}_{\Gamma}$, откуда немедленно вытекает первое требование в условии 2.20. Предложение доказано.

Итак, мы видим, что если выполнено условие 1.2, то для почти всех $\omega$ марковский компакт $X(\omega)$ строго эргодичен, бирегулярен по Ляпунову и сбалансирован. Следовательно, применима лемма 2.30, и мы получаем следующую аппроксимационную теорему для случайных марковских компактов.

ТеоремА 1. Пусть $\mu$ - эргодическая $\sigma$-инвариантная вероятностная мера на $\Omega$, удовлетворяющая условию 1.2. Для любого $\varepsilon>0$ существует такая константа $C_{\varepsilon}$, зависящая только от $\mu$, что при почти всех $\omega \in \Omega$ существует такое непрерывное отображсение $\Xi_{\omega}^{+}: \operatorname{Lip}_{w}^{+}(X(\omega)) \rightarrow \mathfrak{B}^{+}(X(\omega))$, что

1) для любого $t_{0} \in \mathbb{R}$ верно равенство $\Xi_{\bar{\omega}}^{+}\left(f \circ h_{t_{0}}^{+}\right)=\Xi_{\bar{\omega}}^{+}(f)$; 
2) диаграмма

коммутативна;

$$
\begin{array}{crr}
\operatorname{Lip}_{w}^{+}(X(\omega)) & \stackrel{\Xi_{\omega}^{+}}{\longrightarrow} & \mathfrak{B}^{+}(X(\omega)) \\
\uparrow\left(\mathfrak{t}_{\sigma}\right)^{*} & & \downarrow\left(\mathfrak{t}_{\sigma}\right)_{*} \\
\operatorname{Lip}_{w}^{+}(X(\sigma \omega)) & \stackrel{\Xi_{\sigma \omega}^{+}}{\longrightarrow} \mathfrak{B}^{+}(X(\sigma \omega))
\end{array}
$$

3) для любой $f \in \operatorname{Lip}_{w}^{+}(X)$, любого $x \in X(\omega)$ и любого $T>0$

$$
\left|\int_{0}^{T} f \circ h_{t}^{+}(x) d t-\Xi^{+}(f ; x, T)\right| \leqslant C_{\varepsilon}\|f\|_{\operatorname{Lip}_{w}^{+}}\left(1+T^{\varepsilon}\right) .
$$

2.10.5. Гиперболические случайные марковские компакты. Дадим теперь достаточное условие гиперболичности случайных марковских компактов. Пусть $(\mathscr{X}, \mu)$ - вероятностное пространство, на котором задано сохраняющее меру $\mu$ преобразование $T$ или поток $g_{s}$, а также интегрируемый линейный коцикл $A$ над $g_{s}$ (соответственно $T$ ) со значениями в $\mathrm{GL}(m, \mathbb{R})$.

Пусть $E_{0, p}$ - нейтралъное подпространство коцикла $A$ в точке $p \in \mathscr{X}$, т. е. ляпуновское подпространство коцикла $A$, соответствующее нулевому показателю Ляпунова. Будем говорить, что $A$ действует изометрически на своих нейтральных подпространствах, если для почти всех $p$ существует скалярное произведение $\langle\cdot, \cdot\rangle_{p}$ на $\mathbb{R}^{m}$, зависящее измеримым образом от $p$, такое, что

$$
\langle A(1, p) v, A(1, p) v\rangle_{g_{s} p}=\langle v, v\rangle_{p}, \quad v \in E_{0, p},
$$

при всех $s \in \mathbb{R}$ (в случае действия преобразования в последней формуле $g_{s}$ заменяется на $T$ ).

Следующее предложение следует непосредственно из данных определений.

ПредложениЕ 2.36. Пусть $\nu-\sigma$-инвариантная эргодическая вероятностная мера на $\Omega$, удовлетворяющая условию 1.2 , причем ренормализационный коцикл $\mathbb{A}$ действует изометрично на своих нейтралъных подпространствах по отношению $\kappa \nu$. Тогда для почти всех $\omega$ марковский компакт $X(\omega)$ гиперболичен.

Иными словами, любая непрерывная конечно аддитивная мера в действительности должна быть гёльдеровой. Заметим, что условия последнего предложения выполнены, в частности, для символического аналога гладкой меры Мазура-Вича на пространстве модулей абелевых дифференциалов.

\section{Глава 3. Ренормализационный поток на пространстве марковских компактов с мерой}

\section{1. Пространство марковских компактов с мерой}

Пусть $\Omega_{u e} \subset \Omega$ - множество тех $\omega$, для которых марковский компакт $X(\omega)$ строго эргодичен. Для $\omega \in \Omega_{u e}, r \in \mathbb{R}_{+}$определим

$$
\nu_{(\omega, r)}^{+}=\frac{\nu_{\omega}^{+}}{r}, \quad \nu_{(\omega, r)}^{-}=r \nu_{\omega}^{-} .
$$


Ясно, что для любого $r>0$ выполнено равенство

$$
\nu_{\omega}=\nu_{(\omega, r)}^{+} \times \nu_{(\omega, r)}^{-} .
$$

Далее, из определений вытекает, что для $l \in \mathbb{Z}$ выполнено равенство

$$
\left(\mathfrak{t}_{\sigma}\right)_{*}^{l} \nu_{(\omega, r)}^{+}=\nu_{\left(\sigma^{l} \omega, r\left|\lambda_{\omega}^{(l)}\right|\right)}^{+} .
$$

Введем теперь следующее отношение эквивалентности $\sim$ на множестве пар $(\omega, r) \in \Omega_{u e} \times \mathbb{R}_{+}:$

$$
(\omega, r) \sim\left(\omega^{\prime}, r^{\prime}\right)
$$

если существует такое $l \in \mathbb{Z}$, что $\omega^{\prime}=\sigma^{l} \omega, r^{\prime} / r=\left|\lambda^{(l)}(\omega)\right|$.

Поскольку по определению $\lambda_{\sigma^{l} \omega}^{(n)}=\lambda_{\omega}^{(n+l)} /\left|\lambda_{\omega}^{(l)}\right|$, мы получаем

$$
\left|\lambda_{\omega}^{(l)}\right|=\frac{1}{\left|\lambda_{\sigma^{l} \omega}^{(-l)}\right|},
$$

откуда ясно, что действительно является отношением эквивалентности.

\subsection{1. Ренормализационный поток и ренормализационный коцикл.} Пусть $\bar{\Omega}$ - множество классов эквивалентности отношения $\sim$. Введем поток $g_{s}$ на $\bar{\Omega}$ по формуле

$$
g_{s}(\omega, r)=\left(\omega, e^{s} r\right)
$$

Поток $g_{s}$ будем называть ренормализационным потоком.

Фундаментальную область $\Omega_{0}$ отношения $\sim$ можно задать явно:

$$
\Omega_{0}=\left\{(\omega, r): \omega \in \Omega_{u e}, 1 \leqslant r<\left|\lambda^{(1)}(\omega)\right|^{-1}\right\} .
$$

Каждой паре $(\omega, r) \in \Omega_{0}$ поставим в соответствие следующие объекты:

1) марковский компакт $X(\omega, r)=X(\omega)$;

2) слоения $\mathscr{F}_{(\omega, r)}^{+}=\mathscr{F}_{\omega}^{+}, \mathscr{F}_{(\omega, r)}^{-}=\mathscr{F}_{\omega}^{-}$;

3) меры $\nu_{(\omega, r)}^{+}=\nu^{+} / r, \nu_{(\omega, r)}^{-}=r \nu^{+}$;

4) пространства конечно аддитивных мер $\mathfrak{B}_{(\omega, r)}^{+}=\mathfrak{B}_{\omega}^{+}, \mathfrak{B}_{(\omega, r)}^{-}=\mathfrak{B}_{\omega}^{-}$.

Мы отождествляем $\bar{\Omega}$ с $\Omega_{0}$ и для $\bar{\omega} \in \bar{\Omega}$ будем говорить о марковских компактах, $X(\bar{\omega})$, слоениях, мерах и т. д., имея в виду указанные объекты в соответствующей точке $(\omega, r) \in \Omega_{0}$.

Отождествление $\bar{\Omega}$ с $\Omega_{0}$ дает представление $g_{s}$ как специального потока над сдвигом $\sigma$ со специальной функцией

$$
\tau^{1}(\omega)=-\log \left|\lambda^{(1)}(\omega)\right| .
$$

Отметим тот важный факт, что специальная функция $\tau^{1}(\omega)$, задаваемая формулой (18), зависит только от будущего последовательности $\omega$.

При данных $(\omega, r) \in \Omega_{0}$ и $s \in \mathbb{R}$ определим целое число $\widetilde{n}(\omega, r, s)$ по формуле

$$
\left(\omega, e^{s} r\right) \sim\left(\sigma^{\widetilde{n}(\omega, r, s)} \omega, r^{\prime}\right), \quad\left(\sigma^{\widetilde{n}(\omega, r, s)} \omega, r^{\prime}\right) \in \Omega_{0}
$$


Для каждого $s \in \mathbb{R}$ имеется естественное отображение

$$
\mathfrak{t}_{s}: X(\bar{\omega}) \rightarrow X\left(g_{s} \bar{\omega}\right),
$$

задаваемое при $\bar{\omega}=(\omega, r),(\omega, r) \in \Omega_{0}$, формулой $\mathfrak{t}_{s}(\bar{\omega})=\mathfrak{t}_{\sigma}^{\widetilde{n}(\omega, r, s)}$.

Определим пространство

$$
\mathfrak{X} \bar{\Omega}=\{(\bar{\omega}, x), x \in X(\bar{\omega})\}
$$

и зададим на нем поток косого произведения $g_{s}^{\mathfrak{x}}$ по формуле

$$
g_{s}^{\mathfrak{X}}(\bar{\omega}, x)=\left(g_{s}^{\mathfrak{X}} \bar{\omega}, \mathfrak{t}_{s} x\right) .
$$

Непосредственно из определений вытекает, что если $(\omega, r) \sim\left(\omega^{\prime}, r^{\prime}\right)$ и $\omega^{\prime}=\sigma^{l} \omega$, то

$$
\mathfrak{t}_{\sigma}^{l} \nu_{(\omega, r)}^{+}=\nu_{\left(\omega^{\prime}, r^{\prime}\right)}^{+}, \quad \mathfrak{t}_{\sigma}^{l} \nu_{(\omega, r)}^{-}=\nu_{\left(\omega^{\prime}, r^{\prime}\right)}^{-}
$$

и потому

$$
\left(\mathfrak{t}_{s}\right)_{*} \nu_{\bar{\omega}}^{+}=\nu_{g_{s} \bar{\omega}}^{+}, \quad\left(\mathfrak{t}_{s}\right)_{*} \nu_{\bar{\omega}}^{-}=\nu_{g_{s} \bar{\omega}}^{-} .
$$

Для $\bar{\omega}=(\omega, r),(\omega, r) \in \Omega_{0}$, положим

$$
\overline{\mathbb{A}}(s, \bar{\omega})=\mathbb{A}(\widetilde{n}(\omega, r, s), \omega) .
$$

Таким образом, мы получили матричный коцикл $\overline{\mathbb{A}}$ над потоком $g_{s}$.

Пусть $g_{s}^{\overline{\mathbb{A}}}: \bar{\Omega} \times \mathbb{R}^{m} \rightarrow \bar{\Omega} \times \mathbb{R}^{m}$ - отображение косого произведения, связанное с коциклом $\overline{\mathbb{A}}$ формулой

$$
g_{s}^{\overline{\mathbb{A}}}(\bar{\omega}, v)=\left(g_{s} \bar{\omega}, \overline{\mathbb{A}} v\right) .
$$

Как и ранее, при каждом $\bar{\omega}=(\omega, r)$ имеется изоморфизм

$$
\mathscr{I}_{\bar{\omega}}^{+}: \mathbb{R}^{m} \rightarrow \mathfrak{V}^{+}(X(\bar{\omega})),
$$

задаваемый формулой $\mathscr{I}_{\bar{\omega}}^{+} v=\Phi_{\mathbf{v}}^{+}, \mathbf{v}=\left(v^{(n)}\right), v^{(0)}=v$ (напомним, что по определению $\omega \in \Omega_{\mathrm{inv}}$ ). При этом для любого $s \in \mathbb{R}$ коммутативна диаграмма

$$
\begin{array}{lc}
\mathbb{R}^{m} \stackrel{\mathscr{I}_{\bar{\omega}}^{+}}{\longrightarrow} & \mathfrak{V}^{+}(X(\bar{\omega})) \\
\downarrow \mathbb{A}(s, \bar{\omega}) & \left.\downarrow \mathfrak{t}_{s}\right)_{*} \\
\mathbb{R}^{m} \stackrel{\mathscr{I}_{g_{s} \bar{\omega}}^{+}}{\longrightarrow} \mathfrak{V}^{+}\left(X\left(g_{s} \bar{\omega}\right)\right) .
\end{array}
$$

3.1.2. Характеризация конечно аддитивных мер. Определим пространство $\mathfrak{V}^{+} \bar{\Omega}$ как

$$
\mathfrak{V}^{+} \bar{\Omega}=\left\{\left(\bar{\omega}, \Phi^{+}\right), \Phi^{+} \in \mathfrak{V}_{\bar{\omega}}^{+}\right\}
$$

и зададим поток $\mathfrak{T}_{s}$ на нем формулой

$$
\mathfrak{T}_{s}\left(\bar{\omega}, \Phi^{+}\right)=\left(g_{s} \bar{\omega},\left(\mathfrak{t}_{s}\right)_{*} \Phi^{+}\right) .
$$

Тривиализационное отображение

$$
\text { Triv : } \mathfrak{V}^{+} \bar{\Omega} \rightarrow \bar{\Omega} \times \mathbb{R}^{m}
$$


зададим формулой

$$
\operatorname{Triv}\left(\bar{\omega}, \Phi^{+}\right)=\left(\bar{\omega},\left(\mathscr{I}_{\bar{\omega}}^{+}\right)^{-1} \Phi^{+}\right)
$$

Тогда диаграмма

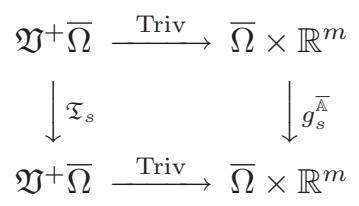

коммутативна.

Напомним, что $W(\mathfrak{G})$ обозначает множество всех конечных слов в алфавите $\mathfrak{G}$ и что для слова $w \in W(\mathfrak{G}), w=w_{0} \ldots w_{n}, w_{i} \in \mathfrak{G}$, символ $\Gamma(w)$ обозначает конкатенацию $w_{0} \ldots w_{n}$.

Напомним, что $\mathfrak{G}_{+}-$множество таких графов $\Gamma \in \mathfrak{G}$, что все элементы матрицы $A(\Gamma)$ положительны. Рассмотрим некоторый граф $\Gamma \in \mathfrak{G}_{+}$и слово $w \in W(\mathfrak{G}), w=w_{0} \ldots w_{n}$, для которого $\Gamma(w)=\Gamma$. Пусть $\mathscr{M}(w, \infty)-$ семейство борелевских эргодических $\sigma$-инвариантных мер $\mu$ на $\Omega$ (конечных или бесконечных), для которых выполнены следующие два условия:

1) $\mu\left(\left\{\omega: \omega_{0}=w_{0}, \ldots, \omega_{n}=w_{n}\right\}\right)>0$;

2) $\int_{\Omega} \tau^{1}(\omega) d \mu(\omega)=1$

Из первого условия и эргодичности меры $\mu$ следует, что $\mu\left(\Omega \backslash \Omega_{u e}\right)=0$. Из второго условия следует, что мера

$$
\mathrm{P}_{\mu}=\mu \times \frac{d r}{r}
$$

является корректно определенной вероятностной $g_{s}$-инвариантной мерой на $\bar{\Omega}$.

Обозначим

$$
\mathscr{M}(\Gamma, \infty)=\bigcup_{w \in W(\mathfrak{G}), \Gamma(w)=\Gamma} \mathscr{M}(w, \infty) .
$$

Пусть $\mathscr{P}^{+}-$пространство эргодических вероятностных $g_{s}$-инвариантых мер Р на $\bar{\Omega}$, имеющих вид $\mathrm{P}=\mathrm{P}_{\mu}, \mu \in \mathscr{M}(\Gamma, \infty), \Gamma \in \mathfrak{G}_{+}$.

По определению, для любой меры $\mathrm{P} \in \mathscr{P}+$ коцикл $\overline{\mathbb{A}}$ интегрируем по меpe $\mathrm{P}$, и теорема Оселедца применима к $\overline{\mathbb{A}}$. Обозначим $E_{\bar{\omega}}^{u}$ строго неустойчивое пространство коцикла $\overline{\mathbb{A}}$ в точке $\bar{\omega} \in \bar{\Omega}$.

ПреДЛОЖенИЕ 3.1. Для почти всех $\bar{\omega}$ отображение $\mathscr{I}_{\bar{\omega}}^{+}$индуцирует изоморфизм строго неустойчивого пространства $E_{\bar{\omega}}^{u}$ кочикла $\overline{\mathbb{A}}$ в точке $\bar{\omega}$ в пространство $\mathfrak{B}^{+}(X(\bar{\omega}))$. 
ДоказАтельство. Начнем со случая, когда мера $\mu$ конечна. В этом случае для $\bar{\omega} \in \bar{\Omega}, \bar{\omega}=(\omega, r)$, неустойчивое пространство $E_{\bar{\omega}}^{u}$ коцикла $\overline{\mathbb{A}}$ в $\bar{\omega}$ совпадает с неустойчивым пространством $E_{\omega}^{u}$ коцикла $\mathbb{A}$ в $\omega$. Производя конкатенацию графов между последовательными появлениями слова $w$ и рассматривая индуцированное отображение для сдвига $\sigma$ на цилиндре $\left\{\omega: \omega_{0}=w_{0}, \ldots, \omega_{n}=w_{n}\right\}$, получаем предложение 3.1 как прямое следствие предложения 2.32 .

В этом случае ляпуновские показатели коциклов $\mathbb{A}$ и $\overline{\mathbb{A}}$ связаны следующим образом.

ПРеДЛОЖЕНИЕ 3.2. Если положительные показатели Ляпунова коцикла $\mathbb{A}-э m o$

$$
\theta_{1}>\theta_{2}>\cdots>\theta_{l_{0}},
$$

то положительные показатели Ляпунова $\bar{\theta}_{i}$ коцикла $\overline{\mathbb{A}}$ задаются формулой

$$
\bar{\theta}_{i}=\frac{\theta_{i}}{\theta_{1}} .
$$

ЗАМЕЧАНИЕ. В частности, всегда выполнено равенство $\bar{\theta}_{1}=1$.

Перейдем к случаю, когда $\mu \in \mathscr{M}(w, \infty)$ бесконечна. Снова рассмотрим индуцированное отображение для сдвига $\sigma$ на цилиндре $\left\{\omega: \omega_{0}=w_{0}, \ldots, \omega_{n}=\right.$ $\left.w_{n}\right\}$ и проверим, что индуцированная мера конечна.

Более точно, пусть $\mathfrak{G}_{\Gamma} \subset \mathfrak{G}$ задано формулой

$$
\mathfrak{G}_{\Gamma}=\left\{\Gamma^{\prime} \in \mathfrak{G}: \Gamma^{\prime}=\Gamma \Gamma^{\prime \prime} \text { для некоторого } \Gamma^{\prime \prime} \in \mathfrak{G}\right\} .
$$

Пусть $\Omega_{\Gamma} \subset \Omega$ - подпространство последовательностей, все символы которых лежат в $\mathfrak{G}_{\Gamma}$, и пусть $\bar{\Omega}_{\Gamma} \subset \bar{\Omega}-$ множество пар $(\omega, r) \in \bar{\Omega}$, для которых $\omega \in \Omega_{\Gamma}$.

Пусть $\mathscr{M}_{\Gamma}$ - пространство борелевских эргодических $\sigma$-инвариантных мер $\mu$ на $\Omega_{\Gamma}$ таких, что

$$
\int_{\Omega_{\Gamma}} \tau^{1}(\omega) d \mu(\omega)=1
$$

Если $\Gamma \in \mathfrak{G}_{+}$, то любая мера в $\mathscr{M}_{\Gamma}$ обязательно конечна, поскольку функция $\tau(\omega)$ отделена от нуля на $\Omega_{\Gamma}$.

Пусть $\Omega^{\prime}(w, \infty) \subset \Omega$ состоит из всех двусторонне бесконечных последовательностей, в которых слово $w$ встречается бесконечно много раз как в прошлом, так и в будущем, и пусть $\Omega(w, \infty) \subset \Omega^{\prime}(w, \infty)$ есть множество $\omega \in \Omega^{\prime}(w, \infty)$, удовлетворяющих дополнительному условию $\omega_{0}=w_{0}, \ldots, \omega_{n}=w_{n}$. Пусть $\sigma(w, \infty)$ - индуцированное отображение для сдвига $\sigma$ на $\Omega(w, \infty)$.

Положим

$$
\bar{\Omega}(w, \infty)=\left\{(\omega, r) \in \bar{\Omega}, \omega \in \Omega^{\prime}(w, \infty)\right\} .
$$

Для меры $\mu \in \mathscr{M}(w, \infty)$ обозначим $\mu(w, \infty)$ ее ограничение на $\Omega(w, \infty)$.

Производя конкатенацию графов между последовательными появлениями слова $w$, мы получаем естественную агрегирующую сюръекцию

$$
\operatorname{Ag}^{w}: \Omega(w, \infty) \rightarrow \Omega_{\Gamma}
$$


для которой диаграмма

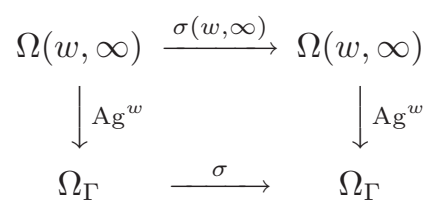

коммутативна.

Отображение $\mathrm{Ag}^{w}$ поднимается до отображения $\overline{\mathrm{Ag}^{w}}: \bar{\Omega}(w, \infty) \rightarrow \bar{\Omega}_{\Gamma}$, которое делает коммутативной диаграмму

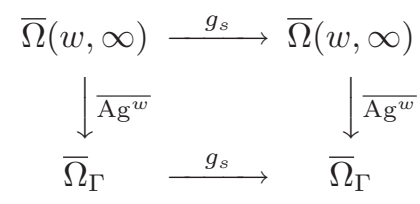

Это отображение $\overline{\mathrm{Ag}^{w}}$ сохраняет коцикл $\overline{\mathbb{A}}$ в следующем смысле: если отображение $\overline{\operatorname{Ag}^{w}} \times \operatorname{Id}: \bar{\Omega}(w, \infty) \times \mathbb{R}^{m} \rightarrow \bar{\Omega}_{\Gamma} \times \mathbb{R}^{m}$ задано формулой

$$
\overline{\operatorname{Ag}^{w}} \times \operatorname{Id}(\bar{\omega}, v)=\left(\overline{\operatorname{Ag}^{w}} \bar{\omega}, v\right),
$$

то диаграмма

коммутативна.

$$
\begin{array}{rc}
\bar{\Omega}(w, \infty) \times \mathbb{R}^{m} \stackrel{g_{s}^{\overline{\mathbb{A}}}}{\longrightarrow} \bar{\Omega}(w, \infty) \times \mathbb{R}^{m} \\
\downarrow \overline{\mathrm{Ag}^{w}} \times \mathrm{Id} & \downarrow \overline{\mathrm{Ag}^{w}} \times \mathrm{Id} \\
\bar{\Omega}_{\Gamma} \times \mathbb{R}^{m} \stackrel{g_{s}^{\overline{\mathbb{A}}}}{\longrightarrow} & \bar{\Omega}_{\Gamma} \times \mathbb{R}^{m}
\end{array}
$$

Для $\mu \in \mathscr{M}(w, \infty)$ положим

$$
\mu^{w}=\left(\operatorname{Ag}^{w}\right)_{*} \mu
$$

Соответствие $\mu \mapsto \mu^{w}$ индуцирует аффинное отображение из $\mathscr{M}(w, \infty)$ в $\mathscr{M}_{\Gamma}$. Из данных определений непосредственно вытекает следующее предложение.

ПрЕДЛОжЕНИЕ 3.3. Для любой меры $\mu \in \mathscr{M}(w, \infty)$ динамические системы $\left(\bar{\Omega}, \mathrm{P}_{\mu}, g_{s}\right)$ u $\left(\bar{\Omega}_{\Gamma}, \mathrm{P}_{\mu^{w}}, g_{s}\right)$ измеримо изоморбны.

Таким образом, случай бесконечной меры сведен к случаю конечной меры, в котором применимо предложение 2.32. Тем самым предложение 3.1 полностью доказано.

3.1.3. Пространство “символических потоков переноса". Предположим теперь, что для почти всех $\omega$ имеется упорядочение Вершика o $(\omega)$ на ребрах каждого графа $\omega_{n}, n \in \mathbb{Z}$. Более того, предположим, что это упорядочение инвариантно относительно сдвига в следующем смысле: упорядочение $\mathfrak{o}(\omega)$ на ребрах графа $\omega_{n+1}$ совпадает с упорядочением $\mathfrak{o}(\sigma \omega)$ на ребрах графа $(\sigma \omega)_{n}=\omega_{n+1}$.

В этом случае для почти всех $\omega \in \Omega$ имеется поток $h_{t}^{+, \omega}$ на $X(\omega)$, а именно, при $(\omega, r) \in \Omega_{0}$ полагаем $h_{t}^{+,(\omega, r)}=h_{t / r}^{+, \omega}$. 
Отождествляя, как и раньше, пространства $\Omega_{0}$ и $\bar{\Omega}$, мы получим, что для почти всех $\bar{\omega}$ задан поток $h_{t}^{+, \bar{\omega}}$ на $X(\bar{\omega})$, так что коммутативна диаграмма

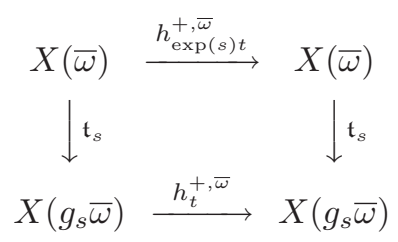

Напомним, что $\mathscr{P}^{+}$есть пространство эргодических вероятностных $g_{s}$-инвариантных мер $\mathrm{P}$ на $\bar{\Omega}$, имеющих вид $\mathrm{P}=\mathrm{P}_{\mu}, \mu \in \mathscr{M}(\Gamma, \infty), \Gamma \in \mathfrak{G}_{+}$. Из теоремы 1 теперь вытекает следующее утверждение.

СледСтвиЕ 3.4. Пусть $\mathrm{P} \in \mathscr{P}^{+}$. Для любого $\varepsilon>0$ существует константа $C_{\varepsilon}$, зависящая только от $\mathrm{P}$, такая, что для почти всех $\bar{\omega} \in \bar{\Omega}$ существует непрерьвное отображение $\Xi_{\bar{\omega}}^{+}: \operatorname{Lip}_{w}^{+}(X(\bar{\omega})) \rightarrow \mathfrak{B}^{+}(X(\bar{\omega}))$, удовлетворяющее следующим условиям:

1) для всех $t_{0} \in \mathbb{R}$ выполнено равенство $\Xi_{\bar{\omega}}^{+}\left(f \circ h_{t_{0}}^{+}\right)=\Xi_{\bar{\omega}}^{+}(f)$;

2) диаграмма

$$
\begin{array}{crr}
\operatorname{Lip}_{w}^{+}(X(\bar{\omega})) & \stackrel{\Xi_{\bar{\omega}}^{+}}{\longrightarrow} & \mathfrak{B}^{+}(X(\bar{\omega})) \\
\uparrow\left(\mathfrak{t}_{s}\right)^{*} & & {\left(\mathfrak{t}_{s}\right)_{*}} \\
\operatorname{Lip}_{w}^{+}\left(X\left(g_{s} \bar{\omega}\right)\right) & \stackrel{\Xi_{g_{s} \bar{\omega}}^{+}}{\longrightarrow} \mathfrak{B}^{+}\left(X\left(g_{s} \bar{\omega}\right)\right)
\end{array}
$$

коммутативна;

3) для любой $f \in \operatorname{Lip}_{w}^{+}(X)$, любого $x \in X(\bar{\omega})$ и любого $T>0$ верна оценка

$$
\left|\int_{0}^{T} f \circ h_{t}^{+}(x) d t-\Xi^{+}(f ; x, T)\right| \leqslant C_{\varepsilon}\|f\|_{\operatorname{Lip}_{w}^{+}}\left(1+T^{\varepsilon}\right) .
$$

\section{2. Предельные теоремы в случае простого второго показателя Ляпунова}

3.2.1. Главный член в асимптотике эргодического интеграла. Предположим, что первый и второй показатели Ляпунова коцикла $\overline{\mathbb{A}}$ являются простыми; напомним, что простота ляпуновского спектра коцикла Концевича-Зорича получена А. Авилой и М. Вианой [4]. Рассмотрим соответствующие подпространства $E_{1, \bar{\omega}}^{u}=\mathbb{R} h_{\bar{\omega}}$ и $E_{2, \bar{\omega}}^{u}$. Пусть также $E_{\geqslant 3, \bar{\omega}}^{u}-$ подпространство, соответствующее оставшимся показателям Ляпунова.

Таким образом, имеет место разложение

$$
E_{\bar{\omega}}^{u}=E_{1, \bar{\omega}}^{u} \oplus E_{2, \bar{\omega}}^{u} \oplus E_{\geqslant 3, \bar{\omega}}^{u} .
$$

Сходное разложение имеется и для $\widetilde{E}^{u}$ :

$$
\widetilde{E}_{\bar{\omega}}^{u}=\widetilde{E}_{1, \bar{\omega}}^{u} \oplus \widetilde{E}_{2, \bar{\omega}}^{u} \oplus \widetilde{E}_{\geqslant 3, \bar{\omega}}^{u} .
$$


Выберем $\Phi_{2}^{+} \in \mathscr{I}_{\bar{\omega}}\left(E_{2, \bar{\omega}}^{u}\right), \Phi_{2}^{-} \in \mathscr{I}_{\bar{\omega}}\left(\widetilde{E}_{2, \bar{\omega}}^{u}\right)$ таким образом, чтобы выполнялось равенство

$$
\left\langle\Phi_{2}^{+}, \Phi_{2}^{-}\right\rangle=1
$$

Возьмем $f \in \operatorname{Lip}_{w}^{+}(X), x \in X, T \in \mathbb{R}$ и заметим, что выражение

$$
m_{\Phi_{2}^{-}}(f) \Phi_{2}^{+}(x, T)
$$

не зависит от выбора конкретных $\Phi_{2}^{ \pm}$(мы можем умножать $\Phi_{2}^{+}$на любой скаляр, одновременно деля $\Phi_{2}^{-}$на тот же скаляр).

Теперь для $f \in \operatorname{Lip}_{w}^{+}(X)$ положим

$$
\Phi_{f}^{+}(x, T)=\left(\int_{X} f d \nu\right) \cdot T+m_{\Phi_{2}^{-}}(f) \Phi_{2}^{+}(x, T)+\Phi_{3, f}^{+}(x, T),
$$

где $\Phi_{3, f}^{+} \in \mathscr{I}_{\bar{\omega}}\left(\widetilde{E}_{\geqslant 3, \bar{\omega}}^{u}\right)$.

В частности, существуют такие положительные константы $C$ и $\alpha$, зависящие только от $\mathrm{P}$, что для любой функции $f \in \operatorname{Lip}_{w}^{+}(X)$ такой, что $\int_{X} f d \nu=0$, выполнено неравенство

$$
\left|\int_{0}^{T} f \circ h_{t}^{+}(x) d t-m_{\Phi_{2}^{-}}(f) \Phi_{2}^{+}(x, T)\right| \leqslant C\|f\|_{\text {Lip }} T^{\bar{\theta}_{2}-\alpha} .
$$

3.2.2. Рост дисперсии. Чтобы оценить дисперсию случайной величины $\int_{0}^{T} f \circ h_{t}^{+}(x) d t$, изучим сначала рост дисперсии случайной величины $\Phi_{2, \bar{\omega}}^{+}(x, T)$ при $T \rightarrow \infty$.

Напомним, что $\mathrm{E}_{\nu(\bar{\omega})} \Phi_{2, \bar{\omega}}^{+}(x, T)=0$ для всех $T$, в то время как

$$
\operatorname{Var}_{\nu(\bar{\omega})} \Phi_{2, \bar{\omega}}^{+}(x, T) \neq 0 \quad \text { для } T \neq 0 .
$$

Отметим также, что выше для коцикла $\Phi^{+} \in \mathfrak{B}_{\bar{\omega}}^{+}, \Phi^{+}=\mathscr{I}_{\bar{\omega}}^{+}(v)$, определена его норма $\left|\Phi^{+}\right|$по формуле $\left|\Phi^{+}\right|=|v|$. Введем мультипликативный коцикл $H_{2}(s, \bar{\omega})$ над потоком $g_{s}$ по формуле

$$
H_{2}(s, \bar{\omega})=\frac{|\overline{\mathbb{A}}(s, \bar{\omega}) v|}{|v|}, \quad v \in E_{2, \bar{\omega}}^{u}, \quad v \neq 0 .
$$

Заметим, что правая часть не зависит от конкретного выбора $v \neq 0$.

По определению мы получаем, что

$$
\lim _{s \rightarrow \infty} \frac{\log H_{2}(s, \bar{\omega})}{s}=\bar{\theta}_{2} .
$$

ПреДЛОжеНИЕ 3.5. Существует такая положительная измеримая функиия $V: \bar{\Omega} \rightarrow \mathbb{R}_{+}$, что для Р-почти всех $\bar{\omega} \in \bar{\Omega}$ верно равенство

$$
\operatorname{Var}_{\nu} \Phi_{2}^{+}(x, T)=V\left(g_{s} \bar{\omega}\right)\left|\Phi_{2}^{+}\right|^{2}\left(H_{2}(s, \bar{\omega})\right)^{2} .
$$


Действительно, функция $V(\bar{\omega})$ задается формулой

$$
V(\bar{\omega})=\frac{\operatorname{Var}_{\nu} \Phi_{2}^{+}(x, 1)}{\left|\Phi_{2}^{+}\right|^{2}}
$$

Заметим, что правая часть не зависит от конкретного выбора $\Phi_{2}^{+} \in \mathfrak{B}_{2, \bar{\omega}}^{+}$, $\Phi_{2}^{+} \neq 0$.

Используя (19), мы переходим теперь к оценке роста дисперсии эргодического интеграла

$$
\int_{0}^{T} f \circ h_{t}^{+}(x) d t
$$

Для $\tau \in[0,1], s \in \mathbb{R}$ и вещественнозначной функции $f \in \operatorname{Lip}_{w, 0}^{+}(X)$ мы определяем

$$
\mathfrak{S}[f, s ; \tau, x]=\int_{0}^{\tau \exp (s)} f \circ h_{t}^{+}(x) d t .
$$

Поскольку $x$ пробегает вероятностное пространство $(X, \nu)$, мы получаем случайный элемент в $C[0,1]$. Иными словами, мы получаем случайную величину

$$
\mathfrak{S}[f, s]:(X, \nu) \rightarrow C[0,1],
$$

определенную по формуле (20).

Для любого фиксированного $\tau \in[0,1]$ формула (20) задает вещественнозначную случайную величину

$$
\mathfrak{S}[f, s ; \tau]:(X, \nu) \rightarrow \mathbb{R}
$$

математическое ожидание которой по определению равно нулю.

ПреДЛОЖЕНИЕ 3.6. Существуют константа $\alpha>0$, зависящая только от $\mathrm{P}$, и положительная измеримая функиия $C: \bar{\Omega} \times \bar{\Omega} \rightarrow \mathbb{R}_{+}$такие, что для Р-почти всех $\bar{\omega} \in \bar{\Omega}$ выполнено следующее. Пусть $\Phi_{2, \bar{\omega}}^{+} \in \mathfrak{B}^{+}, \Phi_{2, \bar{\omega}}^{-} \in \mathfrak{B}^{-}$таковы, что $\left\langle\Phi_{2, \bar{\omega}}^{+}, \Phi_{2, \bar{\omega}}^{-}\right\rangle=1$. Пусть также функиия $f \in \operatorname{Lip}_{w, \bar{\omega}}^{+}$удовлетворяет условию

$$
\int_{X(\bar{\omega})} f d \nu(\bar{\omega})=0, \quad m_{\Phi_{2, \bar{\omega}}^{-}}(f) \neq 0 .
$$

Тогда

$$
\left|\frac{\operatorname{Var}_{\nu(\bar{\omega})} \mathfrak{S}[f, s ; 1]}{V\left(g_{s} \bar{\omega}\right)\left(m_{\Phi_{2}^{-}}(f)\left|\Phi_{2}^{+}\right| H_{2}(s, \bar{\omega})\right)^{2}}-1\right| \leqslant C\left(\bar{\omega}, g_{s} \bar{\omega}\right) e^{-\alpha s} .
$$

ЗАмечАниЕ. Величина $\left(m_{\Phi_{2}^{-}}(f)\left|\Phi_{2}^{+}\right|\right)^{2}$ не зависит от конкретного выбора $\Phi_{2}^{+} \in \mathfrak{B}_{2}^{+}, \Phi_{2}^{-} \in \mathfrak{B}_{2}^{-}$, удовлетворяющих условию $\left\langle\Phi_{2}^{+}, \Phi_{2}^{-}\right\rangle=1$.

Предложение 3.6 непосредственно следует из неравенства

$$
\left|\mathrm{E} \xi_{1}^{2}-\mathrm{E} \xi_{2}^{2}\right| \leqslant \sup \left|\xi_{1}+\xi_{2}\right| \cdot \mathrm{E}\left|\xi_{1}-\xi_{2}\right|
$$

справедливого для любых ограниченных случайных величин $\xi_{1}, \xi_{2}$ на любом вероятностном пространстве, и следующего очевидного предложения. 
ПрЕДЛОЖЕНИЕ 3.7. Существуют константа $\alpha>0$, зависящая только от $\mathrm{P}$, положительная измеримая функиия $C: \bar{\Omega} \times \bar{\Omega} \rightarrow \mathbb{R}_{+}$и положительная измеримая функиия $V^{\prime}: \bar{\Omega} \rightarrow \mathbb{R}_{+}$такие, что

$$
\begin{gathered}
\max \left|\Phi_{2}^{+}\left(x, e^{s}\right)\right|=V^{\prime}\left(g_{s} \bar{\omega}\right) H_{2}(s, \bar{\omega}), \\
\left|\frac{\max \mathfrak{S}[f, s ; 1]}{V^{\prime}\left(g_{s} \bar{\omega}\right)\left(m_{\Phi_{2}^{-}}(f)\left|\Phi^{+}\right| H_{2}(s, \bar{\omega})\right)^{2}}-1\right| \leqslant C\left(\bar{\omega}, g_{s} \bar{\omega}\right) e^{-\alpha s} .
\end{gathered}
$$

3.2.3. Формулировка и доказательство предельной теоремы. Перейдем теперь к изучению асимптотического поведения распределения случайной величины $\mathfrak{S}[f, s]$ при $s \rightarrow \infty$.

Пусть $\mathfrak{m}[f, s]$ - распределение нормализованной случайной величины

$$
\frac{\mathfrak{S}[f, s]}{\sqrt{\operatorname{Var}_{\mathfrak{m}} \mathfrak{S}[f, s ; 1]}} .
$$

Таким образом, мера $\mathfrak{m}[f, s]$ является вероятностным распределением на пространстве $C[0,1]$ непрерывных функций на единичном интервале.

Для $\tau \in \mathbb{R}, \tau \neq 0$, обозначим $\mathfrak{m}[f, s ; \tau]$ распределение вещественнозначной случайной величины

$$
\frac{\mathfrak{S}[f, s ; \tau]}{\sqrt{\operatorname{Var}_{\mathfrak{m}} \mathfrak{S}[f, s ; \tau]}} .
$$

Если $f$ имеет нулевое среднее, то, по своему определению, $\mathfrak{m}[f, s ; \tau]$ является мерой на $\mathbb{R}$ с нулевым математическим ожиданием и единичной дисперсией. Снабдим пространство $C[0,1]$ непрерывных функций на единичном отрезке чебышёвской топологией и определим пространство $\mathfrak{M}=\mathfrak{M}(C[0,1])$ борелевских вероятностных мер на пространстве $C[0,1]$, снабженное слабой топологией (см. [7] или приложение к работе [14]).

Рассмотрим пространство $\bar{\Omega}^{\prime}$, задаваемое формулой

$$
\bar{\Omega}^{\prime}=\left\{\bar{\omega}^{\prime}=(\bar{\omega}, v), v \in E_{2, \bar{\omega}}^{+},|v|=1\right\} .
$$

Поток $g_{s}$ поднимается на $\bar{\Omega}^{\prime}$ :

$$
g_{s}^{\prime}(X, v)=\left(g_{s} X, \frac{\overline{\mathbb{A}}(s, \bar{\omega}) v}{|\overline{\mathbb{A}}(s, \bar{\omega}) v|}\right),
$$

а мера $\mathrm{P}$ при этом поднимается до меры $\mathrm{P}^{\prime}$ на $\bar{\Omega}^{\prime}$. При $\bar{\omega}^{\prime} \in \bar{\Omega}^{\prime}, \bar{\omega}^{\prime}=(\bar{\omega}, v)$, положим

$$
\Phi_{2, \bar{\omega}^{\prime}}^{+}=\mathscr{I}_{\bar{\omega}}(v), \quad V\left(\bar{\omega}^{\prime}\right)=\operatorname{Var}_{\nu(\bar{\omega})} \Phi_{2, \bar{\omega}^{\prime}}^{+}(x, 1) .
$$

Введем отображение

$$
\mathscr{D}_{2}^{+}: \bar{\Omega}^{\prime} \rightarrow \mathfrak{M}
$$

полагая $\mathscr{D}_{2}^{+}\left(\bar{\omega}^{\prime}\right)$ равным распределению $C[0,1]$-значной нормализованной случайной величины

$$
\frac{\Phi_{2, \bar{\omega}^{\prime}}^{+}(x, \tau)}{\sqrt{V\left(\bar{\omega}^{\prime}\right)}}, \quad \tau \in[0,1] .
$$


Отметим, что $\operatorname{Var}_{\nu(\bar{\omega})} \Phi_{2, \bar{\omega}}\left(x, \tau_{0}\right) \neq 0$ для любого $\tau_{0} \neq 0$.

Теперь возьмем функцию $f \in \operatorname{Lip}_{w, \bar{\omega}}^{+}$, удовлетворяющую условию

$$
\int_{X(\bar{\omega})} f d \nu(\bar{\omega})=0, \quad m_{\Phi_{2, \bar{\omega}}^{-}}(f) \neq 0 .
$$

Пусть $d_{\mathrm{LP}}$ обозначает метрику Леви-Прохорова, а $d_{\mathrm{KR}}-$ метрику Канторовича-Рубинштейна на $\mathfrak{M}$ (см. [7] или приложение к работе [14]).

ПРеДЛОЖЕНИЕ 3.8. Пусть для меръ $\mathrm{P} \in \mathscr{P}^{+}$nервый и второй nоказатели Ляпунова ренормализационного коцикла $\overline{\mathbb{A}}$ относительно $\mathrm{P}$ положительны и просты. Тогда существуют положительная измеримая функиия $C$ : $\bar{\Omega} \times \bar{\Omega} \rightarrow \mathbb{R}_{+}$и положительная константа $\alpha$, зависящая только от $\mathrm{P}$, такие, что для Р-почти всех $\bar{\omega}^{\prime} \in \bar{\Omega}^{\prime}, \bar{\omega}^{\prime}=(\bar{\omega}, v)$, и любой $f \in \operatorname{Lip}_{w, 0}^{+}(X)$, для которой $m_{2, X^{\prime}}^{-}(f)>0$, имеют место оценки

$$
\begin{aligned}
& d_{\mathrm{LP}}\left(\mathfrak{m}[f, s], \mathscr{D}_{2}^{+}\left(g_{s}^{\prime} \bar{\omega}^{\prime}\right)\right) \leqslant C\left(\bar{\omega}, g_{s} \bar{\omega}\right) e^{-\alpha s}, \\
& d_{\mathrm{KR}}\left(\mathfrak{m}[f, s], \mathscr{D}_{2}^{+}\left(g_{s}^{\prime} \bar{\omega}^{\prime}\right)\right) \leqslant C\left(\bar{\omega}, g_{s} \bar{\omega}\right) e^{-\alpha s} .
\end{aligned}
$$

ДокАзАтЕЛЬСтво. Начнем с простого неравенства

$$
\left|\frac{a}{b}-\frac{c}{d}\right| \leqslant|a| \cdot\left|\frac{b-d}{b d}\right|+\frac{|a-c|}{d},
$$

справедливого для любых вещественных $a, b, c, d$. Из него следует, что для любой пары случайных величин $\xi_{1}, \xi_{2}$ со значениями в некотором банаховом пространстве и любых положительных чисел $M_{1}, M_{2}$

$$
\sup \left|\frac{\xi_{1}}{M_{1}}-\frac{\xi_{2}}{M_{2}}\right| \leqslant \sup \left|\xi_{1}\right| \cdot\left|\frac{M_{1}-M_{2}}{M_{1} M_{2}}\right|+\frac{\sup \left|\xi_{1}-\xi_{2}\right|}{M_{2}} .
$$

Применим неравенство $(21)$ к $C[0,1]$-значным случайным величинам

$$
\xi_{1}=\mathfrak{S}[f, s], \quad \xi_{2}=\Phi_{2, g_{s} \bar{\omega}}^{+}\left(x, \tau e^{s}\right)
$$

и числам $M_{1}, M_{2}$, равным соответствующим нормализующим дисперсиям: $M_{1}=\operatorname{Var}_{\nu(\bar{\omega})} \mathfrak{S}[f, s ; 1], M_{2}=\operatorname{Var}_{\nu(\bar{\omega})} \mathfrak{m}[f, s ; 1]$.

Теперь заметим, что если для случайных величин $\widetilde{\xi}_{1}, \widetilde{\xi}_{2}$, заданных на вероятностном пространстве $(\Omega, \mathrm{P})$ и принимающих значения в некотором полном метрическом пространстве, расстояние между их значениями не превосходит некоторого $\varepsilon>0$, то и расстояние Леви-Прохорова, и расстояние Канторовича-Рубинштейна между их распределениями $\left(\widetilde{\xi}_{1}\right)_{*} \mathrm{P},\left(\widetilde{\xi}_{2}\right)_{*} \mathrm{P}$ также не превосходят $\varepsilon$ (см. лемму 5.1 в приложении к работе [14]).

Предложение 3.8 теперь немедленно вытекает из (19) и предложения 3.6.

3.2.4. Омега-предельные множества предельных распределений. Для $\bar{\omega}^{\prime} \in \bar{\Omega}^{\prime}$ и $\Phi^{+} \in \mathfrak{B}_{\bar{\omega}}^{+}$обозначим $\mathfrak{m}\left[\Phi^{+}, \tau\right]$ распределение нормализованной вещественнозначной случайной величины

$$
\frac{\Phi^{+}(x, \tau)}{\sqrt{\operatorname{Var}_{\nu} \Phi^{+}(x, \tau)}} .
$$

Тогда из предложения 3.8 получаем такое следствие. 
СлЕДСТВИЕ 3.9. Пусть $\mathrm{P}-g_{s}$-инвариантная эргодическая вероятностная мера на $\bar{\Omega}$ такая, что второй показатель Ляпунова ренормализационного коиикла относительно $\mathrm{P}$ положительный и простой.

Тогда существуют такие два замкнутых множества $\mathfrak{N}_{1}, \mathfrak{N}_{2} \subset \mathfrak{M}$, что для Р-почти всех $\bar{\omega} \in \bar{\Omega}$ и любой функиии $f \in \operatorname{Lip}_{w, 0}^{+}(X(\bar{\omega}))$, для которой $\Phi_{f}^{+} \neq 0$, омега-предельное множество семейства $\mathfrak{m}[f, s]$ совпадает либо с $\mathfrak{N}_{1}$, либо с $\mathfrak{N}_{2}$. Если при этом мера $\mathrm{P}^{\prime}$ эргодична, то $\mathfrak{N}_{1}=\mathfrak{N}_{2}$.

Пусть $\mathfrak{M}(\mathbb{R})$ - пространство борелевских вероятностных мер на $\mathbb{R}$.

ПреДЛОЖЕНИЕ 3.10. Пусть $\mathrm{P} \in \mathscr{P}^{+}$. Для $\mathrm{P}-$ почти всех $\bar{\omega}$ и любой $\Phi^{+} \in$ $\mathfrak{B}_{\bar{\omega}}^{+}, \Phi^{+} \neq 0$, соответствие

$$
\tau \mapsto \mathfrak{m}\left[\Phi^{+}, \tau\right]
$$

задает непрерывное отображение из $\mathbb{R} \backslash\{0\}$ в $\mathfrak{M}(\mathbb{R})$.

ДокАЗАТЕЛЬство. Это утверждение прямо следует из гёльдеровского свойства коцикла $\Phi^{+}$и доказанного в предложении 2.28 факта, состоящего в том, что дисперсия $\operatorname{Var}_{\nu} \Phi^{+}(x, \tau)$ не равна нулю при $\tau \neq 0$.

Как обычно, омега-предельным множеством параметризованной кривой $p(s), s \in \mathbb{R}$, в метрическом пространстве мы называем множество всех точек накопления этой кривой при $s \rightarrow \infty$.

Воспользуемся теперь следующим общим утверждением.

ПрЕДЛОЖЕНИЕ 3.11. Пусть $(\Omega, \mathscr{B})$ - стандартное борелевское пространство, а $g_{s}-$ измеримый поток на $\Omega$, сохраняющий эргодическую борелевскую вероятностную меру $\mu$. Пусть $Z$ - сепарабельное метрическое пространство, и пусть $\varphi: \Omega \rightarrow Z$ - такое измеримое отображение, что для $\mu$-почти всех $\omega \in \Omega$ кривая $\varphi\left(g_{s} \omega\right)$ непрерывна в $s \in \mathbb{R}$. Тогда существует такое замкнутое множество $\mathfrak{N} \subset Z$, что для $\mu$-почти всех $\omega \in \Omega$ множество $\mathfrak{N}$ является омега-предельным множеством кривой $\varphi\left(g_{s} \omega\right), s \in \mathbb{R}$.

ДокАЗАТЕЛЬСтво этого предложения не представляет трудностей. Выберем счетную базу $\mathscr{U}=\left\{U_{n}\right\}_{n \in \mathbb{N}}$ открытых множеств в $Z$. В силу эргодичности $g_{s}$, непрерывности кривых $\varphi\left(g_{s} \omega\right)$ и счетности семейства $\mathscr{U}$, существует такое подмножество полной меры $\Omega^{\prime} \subset \Omega, \mu\left(\Omega^{\prime}\right)=1$, что для любого $U \in \mathscr{U}$ и любого $\omega \in \Omega^{\prime}$ выполнены следующие условия:

1) если $\mu(U)>0$, то существует бесконечная последовательность $s_{n} \rightarrow \infty$ такая, что $\varphi\left(g_{s_{n}} \omega\right) \in U$;

2 ) если $\mu(U)=0$, то существует $s_{0}>0$ такое, что $\varphi\left(g_{s} \omega\right) \notin U$ для всех $s>s_{0}$.

Пусть теперь $\mathfrak{N}$ - множество всех точек $z \in Z$ таких, что $\mu(U)>0$ для любого открытого множества $U \in \mathscr{U}$, содержащего точку $z$. По построению, для любого $\omega \in \Omega^{\prime}$ множество $\mathfrak{N}$ есть в точности омега-предельное множество кривой $\varphi\left(g_{s} \omega\right)$. Предложение доказано.

Предложение 3.11 , примененное к пространству $\Omega^{\prime}$, отображению $\varphi=\mathscr{D}_{2}^{+}$ и эргодической компоненте $\mu$ меры $\mathrm{P}^{\prime}$, совместно с предельной теоремой, даваемой предложением 3.8, немедленно влечет следствие 3.9 . 


\section{3. Предельные теоремы в общем случае}

3.3.1. Формулировка и доказательство предельной теоремы. Перейдем теперь к общему случаю. Пусть $\mathrm{P} \in \mathscr{P}^{+}-$эргодическая $g_{s}$-инвариантная мера на $\bar{\Omega}$, а

$$
\theta_{1}=1>\theta_{2}>\cdots>\theta_{l_{0}}>0
$$

- различные положительные показатели Ляпунова коцикла $\overline{\mathbb{A}}$ по отношению к мере Р. Будем предполагать, что $l_{0} \geqslant 2$.

Для $\bar{\omega} \in \bar{\Omega}$ пусть

$$
E_{\bar{\omega}}^{u}=\mathbb{R} h_{\bar{\omega}}^{(0)}+E_{2, \bar{\omega}} \oplus \cdots \oplus E_{l_{0}, \bar{\omega}}
$$

- соответствующее разложение в прямую сумму оселедцевских подпространств и

$$
\mathfrak{B}_{\bar{\omega}}^{+}=\mathbb{R} \nu_{\bar{\omega}}^{+} \oplus \mathfrak{B}_{2, \bar{\omega}}^{+} \oplus \cdots \oplus \mathfrak{B}_{l_{0}, \bar{\omega}}^{+}
$$

- соответствующее разложение в прямую сумму для пространства $\mathfrak{B} \frac{+}{\omega}$.

Для $f \in \operatorname{Lip}_{w}^{+}(X(\bar{\omega}))$ будем писать

$$
\Phi_{f}^{+}=\Phi_{1, f}^{+}+\Phi_{2, f}^{+}+\cdots+\Phi_{l_{0}, f}^{+},
$$

где $\Phi_{i, f}^{+} \in \mathfrak{B}_{i, \bar{\omega}}^{+}$и, естественно,

$$
\Phi_{1, f}^{+}=\left(\int_{X(\bar{\omega})} f d \nu_{\bar{\omega}}\right) \nu_{\bar{\omega}}^{+} .
$$

Для $i=2, \ldots, l_{0}$ введем измеримое расслоение

$$
\mathbf{S}^{(i)} \bar{\Omega}=\left\{(\bar{\omega}, v): \bar{\omega} \in \bar{\Omega}, v \in E_{i, \bar{\omega}}^{+},|v|=1\right\} .
$$

Поток $g_{s}$ естественным образом поднимается на пространство $\mathbf{S}^{(i)} \bar{\Omega}$ по формуле

$$
g_{s}^{\mathbf{S}^{(i)}}(\bar{\omega}, v)=\left(g_{s} \bar{\omega}, \frac{\mathbb{A}(s, \bar{\omega}) v}{|\mathbb{A}(s, \bar{\omega}) v|}\right) .
$$

Рост нормы векторов $v \in E_{i}^{+}$контролируется мультипликативным коциклом $H_{i}$ над потоком $g_{s}^{\mathbf{S}^{(i)}}$ по формуле

$$
H_{i}(s,(\bar{\omega}, v))=\frac{\mathbb{A}(s, \bar{\omega}) v}{|v|} .
$$

Рост дисперсии эргодических интервалов, как и в предыдущем случае, также описывается коциклом $H_{i}$.

$$
\begin{aligned}
& \text { Для } \bar{\omega} \in \bar{\Omega} \text { и } f \in \operatorname{Lip}_{w, 0}^{+}(X(\bar{\omega})) \text { положим } \\
& \qquad i(f)=\min \left\{j: \Phi_{f, j}^{+} \neq 0\right\} .
\end{aligned}
$$

Определим вектор $v_{f} \in E_{i(f), \bar{\omega}}^{u}$ по формуле

$$
\mathscr{I}_{\bar{\omega}}^{+}\left(v_{f}\right)=\frac{\Phi_{f, i(f)}^{+}}{\left|\Phi_{f, i(f)}^{+}\right|} .
$$


ПРЕДЛОЖЕНИЕ 3.12. Существуют константа $\alpha>0$, зависящая только от $\mathrm{P}$, и положительные измеримые функиии

$$
V^{(i)}: \mathbf{S}^{(i)} \bar{\Omega} \rightarrow \mathbb{R}_{+}, \quad C^{(i)}: \bar{\Omega} \times \bar{\Omega} \rightarrow \mathbb{R}_{+},
$$

где $i=2, \ldots, l_{0}$, такие, что для Р-почти всех $\bar{\omega} \in \bar{\Omega}$, всех $f \in \operatorname{Lip}_{w, 0}^{+}(X(\bar{\omega}))$ $u$ всех $s>0$ выполнено неравенство

$$
\left|\frac{\operatorname{Var}_{\nu(\bar{\omega})} \mathfrak{S}\left[f, e^{s} ; 1\right]}{V^{(i(f))}\left(g_{s}^{\mathbf{S}^{(i)}}\left(\bar{\omega}, v_{f}\right)\right)\left(H_{i}\left(s,\left(\bar{\omega}, v_{f}\right)\right)\right)^{2}}-1\right| \leqslant C^{(i)}\left(\bar{\omega}, g_{s} \bar{\omega}\right) e^{-\alpha s} .
$$

ДокАзАТЕльство. Аналогично случаю простого показателя Ляпунова, для $v \in E_{\bar{\omega}}^{i}$ положим $\Phi_{v}^{+}=\mathscr{I}_{\bar{\omega}}^{+}(v)$ и

$$
V^{(i)}(\bar{\omega}, v)=\operatorname{Var}_{\nu(\bar{\omega})} \Phi_{v}^{+}(x, 1) .
$$

Далее доказательство проводится так же, как и в случае простого второго показателя Ляпунова: если поточечно приблизить эргодический интеграл соответствующим гёльдеровским коциклом, то дисперсии этих случайных величин будут экспоненциально близки. Предложение доказано.

Перейдем теперь к формулировке и доказательству предельной теоремы в общем случае. Для $i=2, \ldots, l_{0}$ определим отображение

$$
\mathscr{D}_{i}^{+}: \mathbf{S}^{(i)} \bar{\Omega} \rightarrow \mathfrak{M}
$$

такое, что $\mathscr{D}_{i}^{+}(\bar{\omega}, v)$ есть распределение $C[0,1]$-значной случайной величины

$$
\frac{\Phi_{v}^{+}(x, \tau)}{\sqrt{\operatorname{Var}_{\nu(\bar{\omega})} \Phi_{v}^{+}(x, 1)}}, \quad \tau \in[0,1] .
$$

Мера $\mathfrak{m}[f, s] \in \mathfrak{M}$, так же как и выше, является распределением $C[0,1]$-значной случайной величины

$$
\frac{\int_{0}^{\tau \exp (s)} f \circ h_{t}^{+}(x) d t}{\sqrt{\operatorname{Var}_{\nu(\bar{\omega})}\left(\int_{0}^{\exp (s)} f \circ h_{t}^{+}(x) d t\right)}}, \quad \tau \in[0,1] .
$$

Напомним, что $\mathscr{P}^{+}$- это пространство эргодических $g_{s}$-инвариантных вероятностных мер $\mathrm{P}$ на $\bar{\Omega}$, имеющих вид $\mathrm{P}=\mathrm{P}_{\mu}, \mu \in \mathscr{M}(\Gamma, \infty), \Gamma \in \mathfrak{G}_{+}$.

Пусть $l_{0}=l_{0}(\mathrm{P})$ равно числу различных показателей Ляпунова меры $\mathrm{P} \in \mathscr{P}^{+}$. Для $f \in \operatorname{Lip}_{w, 0}^{+}(X(\bar{\omega}))$ определим число $i(f)$ согласно $(22)$ и вектор $v_{f}$ согласно $(23)$.

Теорема 2. Пусть $\mathrm{P} \in \mathscr{P}^{+}$. Существуют константа $\alpha>0$, зависящая только от $\mathrm{P}$, и положительное измеримое отображение $C: \bar{\Omega} \times \bar{\Omega} \rightarrow \mathbb{R}_{+}$ такие, что для Р-почти всех $\bar{\omega} \in \bar{\Omega}$ и всех $f \in \operatorname{Lip}_{w, 0}^{+}(X(\bar{\omega}))$ верны неравенства

$$
\begin{aligned}
& d_{\mathrm{LP}}\left(\mathfrak{m}[f, s], D_{i(f)}^{+}\left(g_{s}^{\mathbf{S}^{(i(f))}}\left(\bar{\omega}, v_{f}\right)\right)\right) \leqslant C\left(\bar{\omega}, g_{s} \bar{\omega}\right) e^{-\alpha s}, \\
& d_{\mathrm{KR}}\left(\mathfrak{m}[f, s], D_{i(f)}^{+}\left(g_{s}^{\mathbf{S}^{(i(f))}}\left(\bar{\omega}, v_{f}\right)\right)\right) \leqslant C\left(\bar{\omega}, g_{s} \bar{\omega}\right) e^{-\alpha s} .
\end{aligned}
$$


Доказательство аналогично доказательству предложения 3.8. Как и в том случае, эргодический интеграл равномерно приближается соответствующим коциклом, равномерная оценка разности дает равномерную оценку разности и отношения дисперсий эргодического интеграла и рассматриваемого коцикла. Эргодический интеграл снова равномерно приближается соответствующим коциклом; равномерная оценка разности между эргодическим интегралом и коциклом влечет наличие равномерных оценок для разности и для отношения дисперсий эргодического интеграла и коцикла, рассматриваемых как случайные величины; далее мы, как и выше, используем неравенство (21), где $\xi_{1}=\mathfrak{m}[f, s], \xi_{2}=\Phi_{f, i(f)}^{+}(x, \tau)$, а $M_{1}, M_{2}$ - соответствующие нормализующие дисперсии. Завершается доказательство снова применением того факта, что равномерная оценка для разности двух случайных величин влечет такую же оценку для расстояний Леви-Прохорова и Канторовича-Рубинштейна между распределениями этих случайных величин (см. лемму 5.1 в приложении к работе [14]).

\subsection{2. Атомы предельных распределений.}

ПреДЛОЖЕНИЕ 3.13. Пусть $\omega \in \Omega$ таково, что $\lambda_{1}^{(0, \omega)}>1 / 2$. Тогда существует такое множество $\Pi \subset X(\omega)$, что

1) $\nu_{\omega}(\Pi) \geqslant\left(2 \lambda_{1}^{(0, \omega)}-1\right) h_{1}^{(0, \omega)}$;

2) для всех $\Phi^{+} \in \mathfrak{B}^{+}(X(\omega))$ функиия $\Phi^{+}\left(x, h_{1}^{(0, \omega)}\right)$ постоянна на П.

ДокАЗАтельство. Зафиксируем $\omega$ и не будем далее указывать его в обозначениях. Рассмотрим трансверсаль

$$
I=\left\{x \in X: x_{n}=\min \left\{e: I(e)=F\left(x_{n+1}\right) \text { при всех } n \leqslant 0\right\}\right.
$$

к потоку и разобьем ее на "подотрезки" $I_{k}=\left\{x \in I: I\left(x_{0}\right)=k\right\}, k=1, \ldots, m$.

На трансверсали $I$ задана естественная условная мера $\nu_{I}$, инвариантная относительно отображения первого возвращения на $I$ для потока $h_{t}^{+}$. Мера $\nu_{I}$ задается формулой

$$
\nu_{I}\left(\left\{x \in I: x_{1}=e_{1}, \ldots, x_{n}=e_{n}\right\}\right)=\lambda_{I\left(e_{n}\right)}^{(n+1)},
$$

где $I\left(e_{k}\right)=F\left(e_{k+1}\right), k=1, \ldots, n-1 . \mathrm{B}$ частности, $\nu_{I}\left(I_{k}\right)=\lambda_{k}^{(0)}$. Обозначим для краткости $t_{1}=h_{1}^{(0)}$. По определению, $h_{t_{1}}^{+} I_{1} \subset I$ и

$$
\nu_{I}\left(I_{1} \cap h_{t_{1}}^{+} I_{1}\right) \geqslant 2 \lambda_{1}^{(0)}-1>0 .
$$

Определим теперь множество

$$
\Pi=\left\{h_{\tau}^{+} x, 0<\tau<t_{1}, x \in I_{1}, h_{t_{1}} x \in I_{1}\right\} .
$$

Ясно, что для него выполняется первое утверждение предложения. Докажем второе утверждение. Отметим сначала, что для всех $\Phi^{+} \in \mathfrak{B}^{+}(X(\omega))$ и любого $\tau, 0 \leqslant \tau \leqslant t_{1}$, величина $\Phi^{+}(x, \tau)$ постоянна, когда $x$ пробегает $I_{1}$. 
Зафиксируем $\Phi^{+} \in \mathfrak{B}^{+}(X(\omega))$ и возьмем произвольное $\widetilde{x} \in \Pi$. Пусть $\widetilde{x}=$ $h_{\tau_{1}}^{+} x_{1}$, где $x_{1} \in I_{1}, 0<\tau_{1}<t_{1}$. Тогда $h_{t_{1}-\tau_{1}}^{+} \widetilde{x} \in I_{1}$, откуда

$$
\Phi^{+}\left(h_{t_{1}-\tau_{1}}^{+} \widetilde{x}, \tau_{1}\right)=\Phi^{+}\left(x_{1}, \tau_{1}\right)
$$

И

$$
\begin{aligned}
\Phi^{+}\left(\widetilde{x}, t_{1}\right) & =\Phi^{+}\left(\widetilde{x}, t_{1}-\tau_{1}\right)+\Phi^{+}\left(h_{t_{1}-\tau_{1}}^{+} \widetilde{x}, \tau_{1}\right) \\
& =\Phi^{+}\left(h_{\tau_{1}}^{+} x_{1}, t_{1}-\tau_{1}\right)+\Phi^{+}\left(x_{1}, \tau_{1}\right)=\Phi^{+}\left(x_{1}, t_{1}\right) .
\end{aligned}
$$

Предложение доказано. Рис. 4 иллюстрирует доказательство.

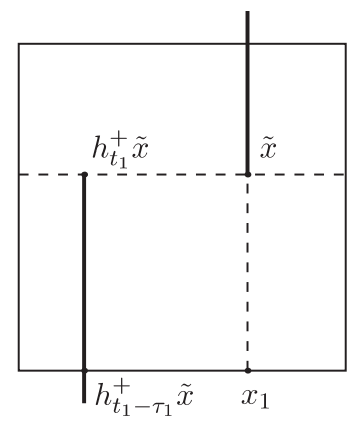

Рис. 4. Атомы предельного распределения

ПРЕДЛОЖЕНИЕ 3.14. Пусть $\widetilde{x}, \widehat{x} \in X$ - различные точки такие, что $\widetilde{x} \in$ $\gamma_{\infty}^{+}(\widehat{x}), \widetilde{x} \in \gamma_{\infty}^{-}(\widehat{x})$, а $t_{0}-$ такое число, что $\widehat{x}=h_{t_{0}}^{+} \widetilde{x}$. Тогда существует такое множество П положительной меры, что для всех $x \in \Pi$ и всех $\Phi^{+} \in \mathfrak{B}^{+}(X)$ верно равенство

$$
\Phi^{+}\left(x, t_{0}\right)=\Phi^{+}\left(\widetilde{x}, t_{0}\right) .
$$

ДокАЗАтельство. Существуют такие $n_{0}, n_{1} \in \mathbb{Z}$, что

$$
\widetilde{x}_{t}=\widehat{x}_{t}, \quad t \in\left(-\infty, n_{0}\right] \cup\left[n_{1}, \infty\right) .
$$

Пусть $\Pi$ - множество всех $x^{\prime}$, удовлетворяющих равенству $x_{t}^{\prime}=\widetilde{x}_{t}$ при $t \in$ $\left(n_{0}, n_{1}\right)$ и равенствам

$$
F\left(x_{n_{1}-1}^{\prime}\right)=F\left(\widetilde{x}_{n_{1}-1}\right), \quad I\left(x_{n_{0}}^{\prime}\right)=I\left(\widetilde{x}_{n_{0}}\right) .
$$

В силу голономной инвариантности для всех $x^{\prime} \in \Pi$ и всех $\Phi^{+} \in \mathfrak{B}^{+}(X)$ верно, чTо

$$
\Phi^{+}\left(x^{\prime}, t_{0}\right)=\Phi^{+}\left(\widetilde{x}, t_{0}\right) .
$$

Чтобы оценить снизу меру множества $\Pi$, положим $I\left(\widetilde{x}_{n_{0}}\right)=i, F\left(\widetilde{x}_{n_{1}-1}\right)=j$ и заметим, что по определению

$$
\nu(\Pi) \geqslant \lambda_{i}^{\left(n_{0}\right)} h_{j}^{\left(n_{1}-1\right)} .
$$

Предложение доказано. 
Для фиксированного $\bar{\omega}$ “гомоклинические моменты времени” $t_{0}$, для которых существуют такие $\widetilde{x}, \widehat{x} \in X$, что $\widetilde{x} \in \gamma_{\infty}^{+}(\widehat{x}), \widetilde{x} \in \gamma_{\infty}^{-}(\widehat{x}), \widehat{x}=h_{t_{0}}^{+} \widetilde{x}$, образуют счетное всюду плотное множество в $\mathbb{R}$.

СледСТВИЕ 3.15. Для почти всех $\bar{\omega} \in \bar{\Omega}$ существует счетное множество таких моментов времени $t_{0} \in \mathbb{R}$, что для любого $\Phi^{+} \in \mathfrak{B}^{+}$распределение случайной величины $\Phi^{+}\left(x, t_{0}\right)$ имеет атом.

3.3.3. Накопление к нулю для предельных распределений. Напомним, что для $\bar{\omega}^{\prime} \in \bar{\Omega}^{\prime}, \Phi^{+} \in \mathfrak{B}_{\bar{\omega}}^{+}, \Phi^{+} \neq 0$, и $\tau \in \mathbb{R}, \tau \neq 0$, мера $\mathfrak{m}\left[\Phi^{+}, \tau\right]$ - это распределение нормализованной вещественнозначной случайной величины

$$
\frac{\Phi^{+}(x, \tau)}{\sqrt{\operatorname{Var}_{\nu} \Phi^{+}(x, \tau)}} .
$$

Как и раньше, пусть $\mathfrak{M}(\mathbb{R})$ - пространство вероятностных мер на $\mathbb{R}$ со слабой топологией; $\delta_{0} \in \mathfrak{M}(\mathbb{R})$ - дельта-мера, сосредоточенная в нуле. Наложим следующее дополнительное условие на меру $\mathrm{P} \in \mathscr{P}^{+}$.

УСловие 3.16. Для любого $\varepsilon>0$

$$
\mathrm{P}\left(\left\{\bar{\omega}: \lambda_{1}^{(\bar{\omega})}>1-\varepsilon, h_{1}^{(\bar{\omega})}>1-\varepsilon\right\}\right)>0 .
$$

По предложению 3.13 , поскольку мера $\mathrm{P}$ эргодична, для почти всех $\bar{\omega} \in \bar{\Omega}$ и любого $\Phi^{+} \in \mathfrak{B}_{\bar{\omega}}^{+}, \Phi^{+} \neq 0$, последовательность мер $\mathfrak{m}\left[\Phi^{+}, \tau\right]$ имеет атомы веса, сколь угодно близкого к 1 . Следующее простое предложение показывает, что соответствующие меры должны тогда накапливаться к нулю (а не к какой-либо другой точке вещественной прямой).

ПреДЛОЖенИЕ 3.17. Пусть $\mu_{0}$ - вероятностная мера на $\mathbb{R}$, для которой

$$
\int_{\mathbb{R}} x d \mu_{0}(x)=0, \quad \int_{\mathbb{R}} x^{2} d \mu_{0}(x)=1 .
$$

Тогда, если $x_{0} \in \mathbb{R} u \mu_{0}\left(\left\{x_{0}\right\}\right)=\beta$, mо

$$
\left|x_{0}\right|^{2} \leqslant \frac{1-\beta}{\beta^{2}} .
$$

ДокАзАтЕльство. Если $x_{0}=0$, то утверждение очевидно, поэтому предположим, что $x_{0}>0$ (случай $x_{0}<0$ получается из этого симметрией). Тогда $\int_{0}^{+\infty} x d \mu_{0}(x) \geqslant \beta x_{0}$, а следовательно, $\int_{-\infty}^{0} x d \mu_{0}(x) \leqslant-\beta x_{0}$.

Используя неравенство Коши-Буняковского-Шварца, получим

$$
\frac{1}{\mu_{0}((-\infty, 0))} \int_{-\infty}^{0} x^{2} d \mu_{0}(x) \geqslant\left(\frac{1}{\mu_{0}((-\infty, 0))} \int_{-\infty}^{0} x d \mu_{0}(x)\right)^{2},
$$

откуда с учетом того, что дисперсия меры $\mu_{0}$ равна 1 , находим, что

$$
\mu_{0}((-\infty, 0)) \geqslant\left(\int_{-\infty}^{0} x d \mu_{0}(x)\right)^{2}
$$

и, окончательно, $1-\beta \geqslant \beta^{2} x_{0}^{2}$, что и требуется доказать. 
Пусть символ $\Rightarrow$ обозначает слабую сходимость вероятностных мер [6], [7].

ПредлОЖениЕ 3.18. Пусть $\mathrm{P} \in \mathscr{P}^{+}-$эргодическая $g_{s}$-инвариантная мера на $\bar{\Omega}$, удовлетворяющая условию 3.16. Тогда для Р-почти всех $X \in \bar{\Omega}$ существует такая последовательность $\tau_{n} \in \mathbb{R}_{+}$, что для любого $\Phi^{+} \in \mathfrak{B}^{+}(X)$ имеет место сходимость

$$
\mathfrak{m}\left[\Phi^{+}, \tau_{n}\right] \Rightarrow \delta_{0} \quad \text { npu } n \rightarrow \infty .
$$

Это предложение непосредственно вытекает из предложений 3.13 и 3.17.

СледСтвиЕ 3.19. Пусть $\mathrm{P} \in \mathscr{P}^{+}$- эргодическая $g_{s}$-инвариантная мера на $\bar{\Omega}$, удовлетворяющая условию 3.16. Тогда для Р-почти всех $X \in \bar{\Omega}$ существует последовательность $s_{n} \in \mathbb{R}_{+}$такая, что для любой функиии $f \in$ $\operatorname{Lip}_{w, 0}^{+}(X)$, для которой $\Phi_{f}^{+} \neq 0$, имеет место сходимость

$$
\mathfrak{m}\left[f, s_{n} ; 1\right] \Rightarrow \delta_{0} \quad \text { npu } n \rightarrow \infty .
$$

Следовательно, если для $f \in \operatorname{Lip}_{w, 0}^{+}(X)$ выполнено $\Phi_{f}^{+} \neq 0$, то семейство мер $\mathfrak{m}[f, s ; 1]$ не сходится при $s \rightarrow \infty$ в слабой топологии на $\mathfrak{M}(\mathbb{R})$, а семейство мер $\mathfrak{m}[f, s]$ не сходится при $s \rightarrow \infty$ в слабой топологии на $\mathfrak{M}(C[0,1])$.

ДокАзАтельство. Первое утверждение следует непосредственно из предложения 3.18 и предельной теоремы 2. Второе утверждение получается из предельной теоремы 2 следующим образом.

Сначала заметим, что множество

$$
\left\{\mathfrak{m}\left[\Phi^{+}, 1\right], \Phi^{+} \in \mathfrak{B}^{+}(X),\left|\Phi^{+}\right|=1\right\}
$$

компактно в слабой топологии (действительно, из равномерной сходимости на сферах в теореме Оселедца ясно, что отображение $\Phi^{+} \mapsto \mathfrak{m}\left[\Phi^{+}, \tau\right]$ непрерывно в ограничении на множество $\left\{\Phi^{+}:\left|\Phi^{+}\right|=1\right\}$, образ которого поэтому компактен). В частности, множество (24) отделено от $\delta_{0}$, и функция

$$
\kappa(\bar{\omega})=\inf _{\Phi^{+}:\left|\Phi^{+}\right|=1} d_{\mathrm{LP}}\left(\mathfrak{m}\left[\Phi^{+}, 1\right], \delta_{0}\right)
$$

является положительной измеримой функцией на $\bar{\Omega}$. Следовательно, существует такое $\kappa_{0}>0$, что

$$
\mathrm{P}\left(\left\{\bar{\omega}: \kappa(\bar{\omega})>\kappa_{0}\right\}\right)>0 .
$$

Из эргодичности меры $\mathrm{P}$ и предельной теоремы 2 следует, что семейство $\mathfrak{m}[f, s ; 1]$, $s \in \mathbb{R}$, не сходится к мере $\delta_{0}$. С другой стороны, как мы видели, мера $\delta_{0}$ является точкой накопления для этого семейства. Следовательно, меры $\mathfrak{m}[f, s ; 1]$ не сходятся в $\mathfrak{M}(\mathbb{R})$ при $s \rightarrow \infty$, а тогда меры $\mathfrak{m}[f, s]$ тем более не сходятся при $s \rightarrow \infty$ в $\mathfrak{M}(C[0,1])$. Следствие доказано.

В случае орициклических потоков на компактных поверхностях постоянной отрицательной кривизны отсутствие сходимости по распределению для нормализованного эргодического интеграла было установлено Л. Фламинио и Дж. Форни [19]. 


\section{4. Эргодические средние для автоморфизмов Вершика}

3.4.1. Пространство односторонних марковских компактов. Напомним, что любой последовательности $\Gamma_{n}, n \in \mathbb{N}$, графов из семейства $\mathfrak{G}$ сопоставляется односторонний марковский компакт, состоящий из всех путей в этой последовательности графов:

$$
Y=\left\{y=y_{1} \ldots y_{n} \ldots, y_{n} \in \mathscr{E}\left(\Gamma_{n}\right), F\left(y_{n+1}\right)=I\left(y_{n}\right)\right\}
$$

Как и раньше, мы обозначаем $A_{n}(Y)=A\left(\Gamma_{n}\right)$.

На марковском компакте $Y$ имеется естественное хвостовое отношение эквивалентности: $y \sim_{\text {tail }} y^{\prime}$, если существует $n_{0}$, для которого $y_{n}=y_{n}^{\prime}$ при всех $n>n_{0}$.

Цилиндры в $Y$ - это подмножества вида $\left\{y: y_{n+1}=e_{1}, \ldots, y_{n+k}=e_{k}\right\}$, где $n \in \mathbb{N}, k \in \mathbb{N}, e_{1} \in \mathscr{E}\left(\Gamma_{n+1}\right), \ldots, e_{k} \in \mathscr{E}\left(\Gamma_{n+k}\right)$ и $F\left(e_{i}\right)=I\left(e_{i+1}\right)$. Совокупность всех цилиндров является полукольцом, которое мы будем обозначать $\mathfrak{C}(Y)$.

Пусть $\mathfrak{V}(Y)$ - векторное пространство всех вещественнозначных конечно аддитивных мер $\Phi$ на полукольце $\mathfrak{C}(Y)$, которые инвариантны относительно хвостового отношения эквивалентности в следующем смысле: если $e_{1} \in$ $\mathscr{E}\left(\Gamma_{1}\right), \ldots, e_{k} \in \mathscr{E}\left(\Gamma_{k}\right)$ и $F\left(e_{i}\right)=I\left(e_{i+1}\right)$, то мера

$$
\Phi\left(\left\{y: y_{1}=e_{1}, \ldots, y_{k}=e_{k}\right\}\right)
$$

зависит только от $I\left(e_{k}\right)$.

Как и раньше, последовательность векторов $\mathbf{v}=v^{(l)}, v^{(l)} \in \mathbb{R}^{m}, l \in \mathbb{N}$, удовлетворяющих равенству

$$
v^{(l)}=A_{l}^{t} v^{(l+1)}
$$

будем называть обратно эквивариантной. По определению, векторное пространство всех обратно эквивариантных последовательностей изоморфно $\mathfrak{V}(Y)$.

Теперь, аналогично случаю бесконечных в обе стороны последовательностей, пусть $\Omega_{+}-$пространство односторонне бесконечных последовательностей графов $\Gamma_{n} \in \mathfrak{G}$. Как и раньше,

$$
\Omega_{+}=\left\{\omega=\omega_{1} \ldots \omega_{n} \ldots, \omega_{i} \in \mathfrak{G}, i \in \mathbb{N}\right\},
$$

и для $\omega \in \Omega_{+}$мы обозначаем $Y(\omega)$ марковский компакт, соответствующий $\omega_{+}$. Левый сдвиг $\sigma$ на пространстве $\Omega$ определяется по формуле $(\sigma \omega)_{n}=\omega_{n+1}$.

Пусть $\mu$ - эргодическая $\sigma$-инвариантная мера на $\Omega_{+}$, естественное продолжение которой на пространство $\Omega$ удовлетворяет условию 1.2. Над системой $\left(\Omega_{+}, \sigma, \mu\right)$ снова возникают два естественных коцикла:

1) ренормализационный коцикл $\mathbb{A}$, определяемый при $n>0$ формулой

$$
\mathbb{A}(n, \omega)=A\left(\omega_{n}\right) \cdots A\left(\omega_{1}\right)
$$

2) обратный транспонированный коцикл $\mathbb{A}^{-t}$, определяемый при $n>0$ формулой

$$
\mathbb{A}^{-t}(n, \omega)=\left(A^{t}\right)^{-1}\left(\omega_{n}\right) \cdots\left(A^{t}\right)^{-1}\left(\omega_{1}\right)
$$


Из сделанных нами предположений следует, что оба этих коцикла удовлетворяют условиям теоремы Оселедца.

Для $\omega \in \Omega_{+}$обозначим $\check{E}_{\omega}$ строго устойчивое ляпуновское подпространство коцикла $\mathbb{A}^{-t}$. Каждому $v \in \check{E}_{\omega}$ соответствует обратно эквивариантная последовательность векторов, а значит, и мера $\Phi_{v} \in \mathfrak{V}(Y)$; обозначим

$$
\mathfrak{B}(\omega)=\mathfrak{B}(Y(\omega))=\left\{\Phi_{v}, v \in \check{E}_{\omega}\right\} .
$$

Пусть $-\theta_{l_{0}}>-\theta_{l_{0}-1}>\cdots>-\theta_{1}-$ различные отрицательные показатели Ляпунова обратного транспонированного коцикла $\mathbb{A}^{-t}$. По теореме Оселедца для почти всех $\omega$ определен соответствующий флаг подпространств

$$
\check{E}_{\theta_{1}} \subset \check{E}_{\theta_{2}} \subset \cdots \subset \check{E}_{\theta_{l_{0}}}
$$

где для любого $v \in \check{E}_{\theta_{i}} \backslash \check{E}_{\theta_{i-1}}$ выполнено соотношение

$$
\lim _{n \rightarrow \infty} \frac{\log \left|\mathbb{A}^{-t}(n, \omega) v\right|}{n}=-\theta_{i}
$$

Обозначим

$$
\mathfrak{B}_{\theta_{i}}(\omega)=\mathfrak{B}_{\theta_{i}}(Y(\omega))=\left\{\Phi_{v}, v \in \check{E}_{\theta_{i}, \omega}\right\} .
$$

Из сделанных предположений, в частности, следует, что для почти всех $\omega$ имеется единственная (с точностью до умножения на скаляр) положительная обратно эквивариантная последовательность; пространство $\mathfrak{V}(Y(\omega))$ содержит тогда единственную положительную счетно аддитивную вероятностную меру $\nu_{\omega}$ и $\nu_{\omega} \in \mathfrak{B}_{\theta_{1}}(Y(\omega))$.

Как и раньше, будем называть ограниченную измеримую функцию $f: Y \rightarrow \mathbb{R}$ слабо липиицевой, если существует такая константа $C>0$, что для любого цилиндра $\mathscr{C} \in \mathfrak{C}(Y)$ и любых точек $y, y^{\prime} \in \mathscr{C}$ выполнено неравенство

$$
\left|f(y)-f\left(y^{\prime}\right)\right| \leqslant C \nu_{\omega}(\mathscr{C})
$$

Если $C_{f}-$ точная нижняя грань всех констант, для которых верно (25), то норма функции $f$ равна

$$
\|f\|_{\operatorname{Lip}_{w}}=C_{f}+\sup _{Y}|f|
$$

Для почти всех $\omega$, всех $f \in \operatorname{Lip}_{w}(Y(\omega))$ и всех $\Phi \in \mathfrak{B}(Y(\omega))$ интеграл Римана-Стилтьеса $\int_{Y} f d \Phi$ корректно определен. Для функции $f \in \operatorname{Lip}_{w}(Y(\omega))$

$$
\theta(f)=\max \left\{\theta_{i}: \text { существует такое } \Phi \in \mathfrak{B}_{\theta_{i}}(Y(\omega)), \text { что } \int_{Y} f d \Phi \neq 0\right\}
$$

(если $\int f d \Phi=0$ для всех $\Phi \in \mathfrak{B}(Y(\omega))$, положим $\left.\theta(f)=0\right)$. 
3.4.2. Автоморфизм Вершика. Как и раньше, будем предполагать, что для почти всех $\omega \in \Omega_{+}$задано упорядочение Вершика $\mathfrak{o}(\omega)$ на ребрах каждого графа $\omega_{n}, n \in \mathbb{N}$. Предположим также, что это упорядочение инвариантно относительно сдвига в следующем смысле: порядок $\mathfrak{o}(\omega)$ на ребрах графа $\omega_{n+1}$ совпадает с порядком $\mathfrak{o}(\sigma \omega)$ на ребрах графа $(\sigma \omega)_{n}=\omega_{n+1}$. Тогда на почти любом марковском компакте $Y(\omega)$ задан автоморфизм Вершика $T_{Y}$ по отношению к упорядочению $\mathfrak{o}(\omega)$ и из теоремы 3.4 вытекает следующее утверждение.

СлЕДСТВИЕ 3.20. Пусть $\mu$ - эргодическая $\sigma$-инвариантная вероятностная мера на $\Omega_{+}$, естественное продолжение которой на пространство $\Omega$ удовлетворяет условию 1.2. Тогда для $\mu$-почти всех $\omega \in \Omega_{+}$, всех $f \in \operatorname{Lip}_{w}(Y(\omega)) u$ всех $у \in Y(\omega)$ верно, что

$$
\limsup _{N \rightarrow \infty} \frac{\log \left|\sum_{k=0}^{N-1} f\left(T_{Y}^{k} y\right)\right|}{\log N}=\theta(f) .
$$

Заметим, что для любого $\omega \in \Omega$ автоморфизм $T_{Y(\sigma \omega)}$ на $Y(\sigma \omega)$ можно следующим образом задать как индуцированный автоморфизм для $T_{Y(\omega)}$ на $Y(\omega)$. Зафиксируем $\Gamma\left(\omega_{1}\right)$ и положим $\mathscr{E}_{\min }\left(\Gamma\left(\omega_{1}\right)\right)$ равным множеству минимальных ребер по отношению к упорядочению o. Рассмотрим его подмножество $Y^{\prime}(\omega) \subset$ $Y(\omega)$ :

$$
Y^{\prime}(\omega)=\left\{x \in Y(\omega): x_{1} \in \mathscr{E}_{\min }\left(\Gamma\left(\omega_{1}\right)\right)\right\}
$$

Сдвиг $\sigma$ биективно отображает $Y^{\prime}(\omega)$ на $Y(\sigma \omega)$; индуцированное отображение $T_{Y(\omega)}$ на $Y^{\prime}(\omega)$ изоморфно $T_{Y(\sigma \omega)}$. Рассмотрим для $\Phi \in \mathfrak{V}(Y(\omega))$ его ограничение $\left.\Phi\right|_{Y^{\prime}(\omega)}$; тогда $\sigma_{*}\left(\left.\Phi\right|_{Y^{\prime}(\omega)}\right) \in \mathfrak{V}(Y(\sigma \omega))$ и если $\Phi \in \mathfrak{B}(Y(\omega))$, то $\sigma_{*}\left(\left.\Phi\right|_{Y^{\prime}(\omega)}\right) \in \mathfrak{B}(Y(\sigma \omega))$.

\section{Глава 4. Марковские компакты и абелевы дифференциалы}

\section{1. Символическое кодирование для потоков на плоских поверхностях}

4.1.1. Перекладывания отрезков как автоморфизмы Вершика. Напомним, что по теореме Вершика [44] любой эргодический автоморфизм вероятностного пространства Лебега можно представить в виде автоморфизма Вершика. Доказательство этой теоремы Вершика [44] производится построением возрастающей последовательности башен Рохлина, которые пересекаются "марковским образом". В случае перекладываний отрезков такая последовательность башен получается, например, при индукции Рози-Вича; в результате мы получаем явное представление минимального перекладывания отрезков в виде автоморфизма Вершика (см. [25]). В следующем п. 4.1.2 мы увидим, что "бесконечный в обе стороны" вариант этой конструкции дает символическое представление для потоков на плоских поверхностях. 
Пусть $\pi$ - неприводимая перестановка $m$ символов, а $\mathbf{T}:[0,1) \rightarrow[0,1)-$ минимальное перекладывание $m$ отрезков с перестановкой $\pi$.

Существует такая последовательность отрезков $I^{(n)}=\left[0, b^{(n)}\right), n=0,1, \ldots$, что

1) $\lim _{n \rightarrow \infty} b^{(n)}=0$;

2) $I^{(n+1)} \subset I^{(n)}$;

3) отображение первого возвращения на $I^{(n)}$ для $\mathbf{T}$ является снова перекладыванием $m$ отрезков.

Пусть $\mathbf{T}_{n}$ - отображение первого возвращения для $\mathbf{T}$ на $I^{(n)}$, а $I_{1}^{(n)}, \ldots, I_{m}^{(n)}$, где $I_{i}^{(n)}=\left[a_{i}^{(n)}, b_{i}^{(n)}\right),-$ подотрезки, которые переставляет $\mathbf{T}_{n}$. По определению, $a_{1}^{(n)}=0, b_{i}^{(n)}=a_{i+1}^{(n)}$.

Представим теперь $I^{(n)}$ как объединение башен Рохлина над $I^{(n+1)}$ относительно отображения $\mathbf{T}_{n}$ :

$$
I^{(n)}=\bigsqcup_{i=1}^{m} \bigsqcup_{k=0}^{N_{i}^{(n+1)}-1} \mathbf{T}_{n}^{k} I_{i}^{(n+1)} .
$$

Здесь $N_{i}^{(n+1)}$ - высота башни, т. е. время первого возвращения $I_{i}^{(n+1)}$ в $I^{(n)}$ под действием $\mathbf{T}_{n}$.

Пусть

$$
\mathscr{E}_{n+1}=\left\{(i, k): i \in\{1, \ldots, m\}, k \in\left\{0, \ldots, N_{i}^{(n+1)}-1\right\}\right\} .
$$

Положим при $e=(i, k) \in \mathscr{E}_{n+1}$

$$
J_{e}^{(n+1)}=\mathbf{T}_{n+1}^{k} I_{i}^{(n+1)} .
$$

Для любого ребра $e \in \mathscr{E}_{n+1}$ существует единственное $j \in\{1, \ldots, m\}$ такое, что

$$
J_{e}^{(n+1)} \subset I_{j}^{(n)} .
$$

Обозначим $j=F(e)$. Будем также писать $i=I(e)$, если $e=(i, k)$. Тогда

$$
I_{j}^{(n)}=\bigsqcup_{e \in \mathscr{E}_{n+1}: \mathscr{F}(e)=j} J_{e}^{(n+1)} .
$$

Представим теперь $I=[0,1)$ как объединение башен Рохлина над $I^{(n)}$ относительно T:

$$
I=\bigsqcup_{i=1}^{m} \bigsqcup_{k=0}^{L_{i}^{(n+1)}-1} \mathbf{T}^{k} I_{i}^{(n)}
$$

Подставляя (26) в (27), мы получим, что

$$
I=\bigsqcup_{i=1}^{m} \bigsqcup_{k=0}^{L_{i}^{(n+1)}-1} \bigsqcup_{e \in \mathscr{E}_{n+1}: F(e)=j} \mathbf{T}^{k} J_{e}^{(n+1)} .
$$


Обозначим полученное разбиение $I$ на подотрезки как $\xi_{n+1}$.

По определению, максимальная длина элементов $\xi_{n}$ стремится к нулю при $n \rightarrow \infty$, поэтому возрастающая последовательность разбиений $\xi_{n}$ стремится (в смысле Рохлина) к разбиению на точки. Как обычно, будем обозначать $\xi_{n}(x)$ элемент разбиения $\xi_{n}$, которому принадлежит точка $x \in I$.

Определим функции $\mathfrak{i}_{l}: I \rightarrow \mathscr{E}_{n}$, положив $\mathfrak{i}_{l}(x)=e$, если $\xi_{l}(x)$ имеет вид $\mathbf{T}^{l} J_{e}^{(l)}$. Будем называть конечную строку $\left(e_{1}, \ldots, e_{n}\right), e_{l} \in \mathscr{E}_{l}$, допустимой, если $F\left(e_{l}\right)=I\left(e_{l-1}\right)$.

ПреДЛОжЕНИЕ 4.1. Пустъ $\left(e_{1}, \ldots, e_{n}\right), e_{l} \in \mathscr{E}_{l},-$ допустимая строка. Тогда существует единственный интервал $J=J\left(e_{1}, \ldots, e_{n}\right)$ такой, что

1) $J$ - элемент разбиения $\xi_{n}$;

2) для любого $x \in J$ верно $\mathfrak{i}_{l}(x)=e_{l}, l=1, \ldots, n$.

Обратно, любой элемент разбиения $\xi_{n}$ имеет вид $J\left(e_{1}, \ldots, e_{n}\right)$ для единственной допустимой строки $\left(e_{1}, \ldots, e_{n}\right)$.

ДоКАЗАТЕЛЬСТВо этого предложения легко проводится по индукции.

Рассмотрим марковский компакт

$$
Y=\left\{y=y_{1} \ldots y_{n} \ldots: y_{i} \in \mathscr{E}_{i}, F\left(y_{i}\right)=I\left(y_{i-1}\right)\right\}
$$

Тогда имеется естественное отображение $p: Y \rightarrow[0,1]$, переводящее $y \in Y$ в точку

$$
\bigcap_{n=1}^{\infty} \overline{J\left(y_{1}, \ldots, y_{n}\right)}
$$

(здесь $\bar{J}$ обозначает замыкание $J$ ). Отображение $p$ сюръективно, а также биективно всюду, кроме концов отрезков $J=J\left(e_{1}, \ldots, e_{n}\right)$ : все они, за исключением 0 и 1 , имеют два прообраза. В частности, отображение $p$ является почти наверное биективным относительно лебеговой меры на $[0,1]$; по определению, образ $\nu_{Y}$ меры Лебега на $[0,1]$ под действием $p^{-1}$ является марковской мерой на $Y$.

Марковский компакт $Y$ обладает также следующей дополнительной структурой. Каждое множество $\mathscr{E}_{n}$ частично упорядочено: пусть $e_{1}, e_{2} \in \mathscr{E}_{n}, e_{1}=$ $\left(i_{1}, k_{1}\right), e_{2}=\left(i_{2}, k_{2}\right)$, будем считать, что $e_{1}<e_{2}$, если $i_{1}=i_{2}, k_{1}<k_{2}$.

Этот порядок порождает частичный порядок о на $Y: y<\widetilde{y}$, если существует $n_{0}$, для которого $y_{n}=\widetilde{y}_{n}$ при всех $n>n_{0}$ и $y_{n_{0}}<\widetilde{y}_{n_{0}}$.

Отображение $p^{-1} \circ \mathbf{T} \circ p: Y \rightarrow Y$ является автоморфизмом Вершика на $Y$ относительно частичного порядка o (см. [36], [44], [46]).

ЗАмЕчАНИЕ. О связи асимптотики эргодических сумм перекладываний и существования блуждающих интервалов обобщенных перекладываний см. работы [10], [32]; о связи со спектром перекладываний см. [3], [35]. 


\subsection{2. Потоки на плоских поверхностях как символические пото-} ки. Пусть $(M, \omega)$ - абелев дифференциал, для которого оба потока $h_{t}^{+}$и $h_{t}^{-}$ минимальны. Прямоугольник П $\left(x, t_{1}, t_{2}\right)=\left\{h_{\tau_{1}}^{+} h_{\tau_{2}}^{-} x, 0 \leqslant \tau_{1}<t_{1}, 0 \leqslant \tau_{2}<t_{2}\right\}$ будем называть слабо допустимым, если для всех достаточно малых $\varepsilon>0$ прямоугольник $\Pi\left(h_{\varepsilon}^{+} h_{\varepsilon}^{-} x, t_{1}-\varepsilon, t_{2}-\varepsilon\right)$ не содержит нулей $\omega$.

Существует такая последовательность разбиений

$$
M=\Pi_{1}^{(n)} \sqcup \cdots \sqcup \Pi_{m}^{(n)}, \quad n \in \mathbb{Z},
$$

что все их элементы $\Pi_{i}^{(n)}$ являются слабо допустимыми прямоугольниками и для любых $n_{1}, n_{2} \in \mathbb{Z}$ и $i_{1}, i_{2} \in\{1, \ldots, m\}$ прямоугольники $\Pi_{i_{1}}^{\left(n_{1}\right)}$ и $\Pi_{i_{2}}^{\left(n_{2}\right)}$ пересекаются "марковским образом" в следующем точном смысле.

Границу слабо допустимого прямоугольника $\Pi\left(x, t_{1}, t_{2}\right)$ разобьем на четыре множества:

$$
\begin{array}{ll}
\partial_{h}^{1}(\Pi)=\overline{\left\{h_{t_{1}}^{+} h_{\tau_{2}}^{-} x, 0 \leqslant \tau_{2}<t_{2}\right\}}, & \partial_{h}^{0}(\Pi)=\overline{\left\{h_{\tau_{2}}^{-} x, 0 \leqslant \tau_{2}<t_{2}\right\}}, \\
\partial_{v}^{1}(\Pi)=\overline{\left\{h_{t_{2}}^{-} h_{\tau_{1}}^{+} x, 0 \leqslant \tau_{1}<t_{1}\right\}}, & \partial_{v}^{0}(\Pi)=\overline{\left\{h_{\tau_{1}}^{+} x, 0 \leqslant \tau_{1}<t_{1}\right\}} .
\end{array}
$$

Условие марковости состоит тогда в том, что для всех $n \in \mathbb{Z}$ и $i \in\{1, \ldots, m\}$ существуют $i_{1}, i_{2}, i_{3}, i_{4} \in\{1, \ldots, m\}$ такие, что

$$
\begin{array}{lll}
\partial_{h}^{1}\left(\Pi_{i}^{(n)}\right) \subset \partial_{h}^{1} \Pi_{i_{1}}^{(n-1)}, & \partial_{h}^{0}\left(\Pi_{i}^{(n)}\right) \subset \partial_{h}^{0} \Pi_{i_{2}}^{(n-1)}, \\
\partial_{v}^{1}\left(\Pi_{i}^{(n)}\right) \subset \partial_{v}^{1} \Pi_{i_{3}}^{(n+1)}, & \partial_{v}^{0}\left(\Pi_{i}^{(n)}\right) \subset \partial_{v}^{0} \Pi_{i_{4}}^{(n+1)} .
\end{array}
$$

Обозначим $\left|\partial_{h}(\Pi)\right|=t_{2},\left|\partial_{v}(\Pi)\right|=t_{1}$ длины сторон слабо допустимого прямоугольника $\Pi=\Pi\left(x, t_{1}, t_{2}\right)$. Будем требовать, чтобы выполнялись соотношения

$$
\lim _{n \rightarrow \infty} \max _{i=1, \ldots, m}\left|\partial_{v} \Pi_{i}^{(n)}\right|=0, \quad \lim _{n \rightarrow \infty} \max _{i=1, \ldots, m}\left|\partial_{h} \Pi_{i}^{(-n)}\right|=0 .
$$

Последовательность разбиений (29), удовлетворяющих марковскому условию и условию (30), существует в силу минимальности вертикального и горизонтального слоений; эта последовательность позволяет нам отождествить поверхность $M$ с пространством путей в неавтономной топологической марковской цепи.

Действительно, пусть $\mathscr{E}_{n}$, где $n \in \mathbb{Z},-$ множество связных компонент пересечений $\Pi_{i}^{(n)} \cap \Pi_{j}^{(n-1)}$. Если $e$ - такая компонента, будем писать $i=I(e), j=F(e)$ (см. рис. 5).

Рассмотрим теперь двусторонний марковский компакт

$$
X=\left\{x=\ldots x_{-n} \ldots x_{n} \ldots, x_{n} \in \mathscr{E}_{n}, F\left(x_{n}\right)=I\left(x_{n-1}\right), n \in \mathbb{Z}\right\}
$$

Для данной точки $x \in X$ рассмотрим пересечение замыканий соответствующих связных компонент:

$$
\xi(x)=\bigcap_{n \in \mathbb{Z}} \overline{x_{n}}
$$

Непустота этого пересечения вытекает из условия марковости, а из условия (30) следует, что пересечение (31) состоит из единственной точки. 

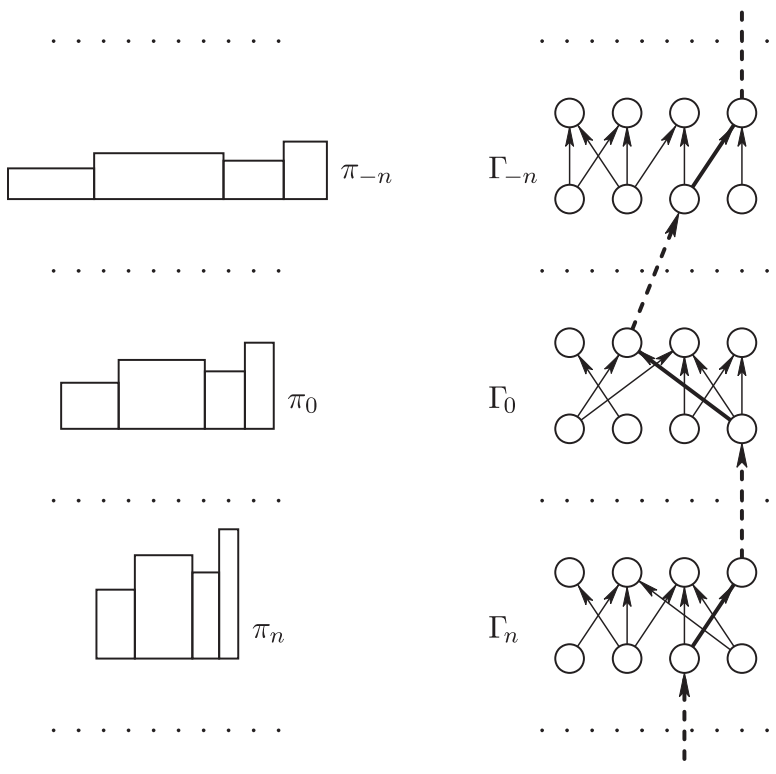

Рис. 5. Ребра графа - это связные компоненты пересечений прямоугольников

Таким образом, мы получили измеримое отображение $\xi: X \rightarrow M$. Ясно, что почти все по мере Лебега точки поверхности $M$ имеют ровно один прообраз, и потому это отображение отождествляет $M$ и $X$ с точностью до множеств меры нуль. Это соответствие между $M$ и $X$ проиллюстрировано на рис. 6 .
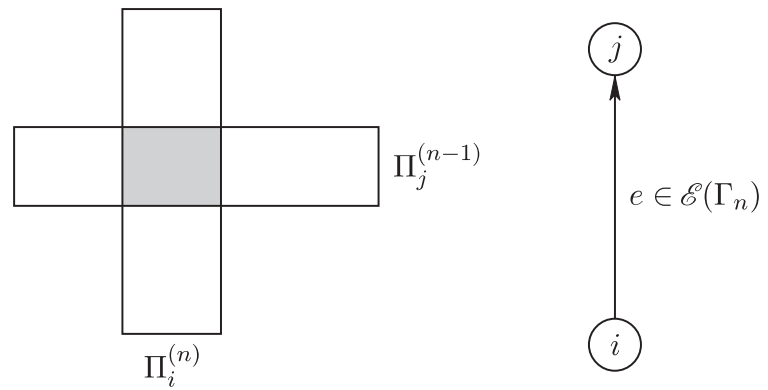

Рис. 6. От абелева дифференциала к марковскому компакту

По построению мера Лебега на поверхности $M$ индуцирует марковскую меру на компакте $X$. Кроме того, ясно, что верно следующее предложение.

ПреДЛОЖЕНИЕ 4.2. Пусть $n_{0} \in \mathbb{Z}$. Если для $x, x^{\prime}$ равенство $x_{n}=x_{n}^{\prime}$ имеет место при всех $n \geqslant n_{0}$, то $\xi(x)$ u $\xi\left(x^{\prime}\right)$ принадлежат одной орбите потока $h_{t}^{+}$; если же для $x, x^{\prime}$ равенство $x_{n}=x_{n}^{\prime}$ имеет место при всех $n \leqslant n_{0}$, то $\xi(x)$ и $\xi\left(x^{\prime}\right)$ лежат на одной орбите потока $h_{t}^{-}$. 
Иными словами, горизонтальный и вертикальный потоки абелева дифференциала $\omega$ соответствуют потокам вдоль асимптотических слоений марковского компакта. Как будет видно далее, гёльдеровские коциклы соответствуют специальным конечно аддитивным мерам на листах этих асимптотических слоений.

\section{2. Пространство зашнурованных прямоугольников Вича}

4.2.1. Индукция Рози-Вича. Чтобы установить связь между марковскими компактами и абелевыми дифференциалами, будем использовать символьное кодирование перекладываний отрезков, даваемое индукцией Рози-Вича, и представления Вича абелевых дифференциалов с помощью зашнурованных прямоугольников. Другой вариант формализма Рози-Вича приводится в работе С. Марми, П. Муссы и Ж.-К. Йоккоза [31].

Пусть $\pi$ - перестановка $m$ символов, причем мы будем всегда считать ее неприводимой в следующем смысле: равенство $\pi\{1, \ldots, k\}=\{1, \ldots, k\}$ имеет место только при $k=m$. Операции Рози $a$ и $b$ определяются формулами

$$
\begin{aligned}
& a \pi(j)= \begin{cases}\pi j, & \text { если } j \leqslant \pi^{-1} m, \\
\pi m, & \text { если } j=\pi^{-1} m+1, \\
\pi(j-1), & \text { если } \pi^{-1} m+1<j \leqslant m ;\end{cases} \\
& b \pi(j)= \begin{cases}\pi j, & \text { если } \pi j \leqslant \pi m, \\
\pi j+1, & \text { если } \pi m<\pi j<m, \\
\pi m+1, & \text { если } \pi j=m .\end{cases}
\end{aligned}
$$

Эти операции переводят неприводимую подстановку снова в неприводимую. Класс Рози $\mathscr{R}(\pi)$ - это множество всех перестановок, которые можно получить из $\pi$ применением группы преобразований, порождаемой $a$ и $b$. Фиксируем далее некоторый класс Рози $\mathscr{R}$ неприводимых подстановок.

Будем при $i, j=1, \ldots, m$ обозначать $E^{i j}$ матрицу размера $m \times m$, в которой $(i, j)$-й элемент равен 1 , а все остальные равны нулю. Также будем обозначать символом $E$ единичную $(m \times m)$-матрицу. Следуя У. Вичу [37], определим следующие унимодулярные матрицы:

$$
\begin{aligned}
& \mathscr{A}(a, \pi)=\sum_{i=1}^{\pi^{-1} m} E^{i i}+E^{m, \pi^{-1} m+1}+\sum_{i=\pi^{-1} m}^{m-1} E^{i, i+1}, \\
& \mathscr{A}(b, \pi)=E+E^{m, \pi^{-1} m}
\end{aligned}
$$

Если $\lambda=\left(\lambda_{1}, \ldots, \lambda_{m}\right) \in \mathbb{R}^{m}$, обозначим

$$
|\lambda|=\sum_{i=1}^{m} \lambda_{i} .
$$

Пусть

$$
\Delta_{m-1}=\left\{\lambda \in \mathbb{R}^{m}:|\lambda|=1, \lambda_{i}>0 \text { при } i=1, \ldots, m\right\} .
$$


Каждой паре $(\lambda, \pi), \lambda \in \Delta_{m-1}$, поставим в соответствие перекладъвание отрезков на полуинтервале $I:=[0,1)$, определяемое следующим образом. Разобьем $I$ на подотрезки $I_{k}:=\left[\beta_{k-1}, \beta_{k}\right)$, где $\beta_{0}=0, \beta_{k}=\sum_{i=1}^{k} \lambda_{i}, 1 \leqslant k \leqslant m$, и разместим $I_{k}$ на отрезке $I$ слева направо в следующем порядке: $I_{\pi^{-1}}, \ldots, I_{\pi^{-1} m}$. Мы получили кусочно линейное отображение полуинтервала $I$ на себя, сохраняющее меру Лебега.

Пространство $\Delta(\mathscr{R})$ перекладываний отрезков, соответствующее классу Рози $\mathscr{R}$, определяется формулой

$$
\Delta(\mathscr{R})=\Delta_{m-1} \times \mathscr{R}
$$

Обозначим

$$
\begin{array}{rlrl}
\Delta_{\pi}^{+} & =\left\{\lambda \in \Delta_{m-1}: \lambda_{\pi^{-1} m}>\lambda_{m}\right\}, & \Delta_{\pi}^{-} & =\left\{\lambda \in \Delta_{m-1}: \lambda_{m}>\lambda_{\pi^{-1} m}\right\}, \\
\Delta^{+}(\mathscr{R}) & =\bigcup_{\pi \in \mathscr{R}}\{\pi\} \times \Delta_{\pi}^{+}, & \Delta^{-}(\mathscr{R}) & =\bigcup_{\pi \in \mathscr{R}}\{\pi\} \times \Delta_{\pi}^{-}, \\
\Delta^{ \pm}(\mathscr{R})=\Delta^{+}(\mathscr{R}) \cup \Delta^{-}(\mathscr{R}) . &
\end{array}
$$

Положим

$$
\mathscr{A}(\lambda, \pi)= \begin{cases}\mathscr{A}(a, \pi), & \text { если } \lambda \in \Delta_{\pi}^{+} ; \\ \mathscr{A}(b, \pi), & \text { если } \lambda \in \Delta_{\pi}^{-} .\end{cases}
$$

Тогда отображсние индукиии Рози-Вича $\mathscr{T}: \Delta^{ \pm}(\mathscr{R}) \rightarrow \Delta(\mathscr{R})$ определяется так:

$$
\mathscr{T}(\lambda, \pi)=\left(\frac{\mathscr{A}(\lambda, \pi)^{-1} \lambda}{\left|\mathscr{A}(\lambda, \pi)^{-1} \lambda\right|}, c \pi\right), \quad \text { где } \quad c= \begin{cases}a, & \text { если } \lambda \in \Delta_{\pi}^{+} \\ b, & \text { если } \lambda \in \Delta_{\pi}^{-}\end{cases}
$$

Можно проверить, что $\mathscr{T}(\lambda, \pi)$ - это перекладывание отрезков, являющееся отображением первого возвращения для перекладывания $(\lambda, \pi)$ на отрезок $J=$ $[0,1-\gamma)$, где $\gamma=\min \left(\lambda_{m}, \lambda_{\pi^{-1} m}\right)$; отрезок $J$ затем нужно растянуть обратно до отрезка единичной длины.

Обозначим

$$
\Delta^{\infty}(\mathscr{R})=\bigcap_{n \geqslant 0} \mathscr{T}^{-n} \Delta^{ \pm}(\mathscr{R})
$$

Любая $\mathscr{T}$-инвариантная вероятностная мера сосредоточена на $\Delta^{\infty}(\mathscr{R})$. С другой стороны, естественная мера Лебега на $\Delta(\mathscr{R})$, которая конечна, но не инвариантна, также сосредоточена на $\Delta^{\infty}(\mathscr{R})$. У. Вич [37] показал, что $\mathscr{T}$ имеет абсолютно непрерывную эргодическую инвариантную меру на $\Delta(\mathscr{R})$, которая, однако, бесконечна.

Над отображением $\mathscr{T}$ задано два матричных коцикла $-\mathscr{A}^{t}$ и $\mathscr{A}^{-1}$ :

$$
\begin{aligned}
\mathscr{A}^{t}(n,(\lambda, \pi)) & =\mathscr{A}^{t}\left(\mathscr{T}^{n}(\lambda, \pi)\right) \cdots \mathscr{A}^{t}(\lambda, \pi), \\
\mathscr{A}^{-1}(n,(\lambda, \pi)) & =\mathscr{A}^{-1}\left(\mathscr{T}^{n}(\lambda, \pi)\right) \cdots \mathscr{A}^{-1}(\lambda, \pi) .
\end{aligned}
$$


Определим соответствующие отображения косых произведений $\mathscr{T}^{\mathscr{A}^{t}}: \Delta(\mathscr{R}) \times$ $\mathbb{R}^{m} \rightarrow \Delta(\mathscr{R}) \times \mathbb{R}^{m}, \mathscr{T}^{\mathscr{A}^{-1}}: \Delta(\mathscr{R}) \times \mathbb{R}^{m} \rightarrow \Delta(\mathscr{R}) \times \mathbb{R}^{m}:$

$$
\begin{aligned}
\mathscr{T}^{\mathscr{A}^{t}}((\lambda, \pi), v) & =\left(\mathscr{T}(\lambda, \pi), \mathscr{A}^{t}(\lambda, \pi) v\right), \\
\mathscr{T}^{\mathscr{A}^{-1}}((\lambda, \pi), v) & =\left(\mathscr{T}(\lambda, \pi), \mathscr{A}^{-1}(\lambda, \pi) v\right) .
\end{aligned}
$$

4.2.2. Построение зашнурованных прямоугольников. Вкратце напомним конструкцию Вича пространства зашнурованных прямоугольников. Мы будем использовать обозначения из [11], [15].

Зашнурованные прямоуголъники, сопоставленные классу Рози $\mathscr{R},-$ это тройки $\mathscr{X}=(\lambda, \pi, \delta)$, где $\lambda=\left(\lambda_{1}, \ldots, \lambda_{m}\right) \in \mathbb{R}^{m}, \lambda_{i}>0, \pi \in \mathscr{R}, \delta=\left(\delta_{1}, \ldots, \delta_{m}\right) \in \mathbb{R}^{m}$ и вектор $\delta$ удовлетворяет следующим неравенствам:

$$
\begin{aligned}
\delta_{1}+\cdots+\delta_{i} \leqslant 0, & i=1, \ldots, m-1, \\
\delta_{\pi^{-1} 1}+\cdots+\delta_{\pi^{-1} i} \geqslant 0, & i=1, \ldots, m-1 .
\end{aligned}
$$

Множество всех векторов $\delta$, удовлетворяющих (34), (35), является конусом в $\mathbb{R}^{m}$, который мы будем обозначать $K(\pi)$.

Для всех $j=1, \ldots, m$ положим

$$
a_{j}=a_{j}(\delta)=-\sum_{i=1}^{j} \delta_{i}, \quad h_{j}=h_{j}(\pi, \delta)=-\sum_{i=1}^{j-1} \delta_{i}+\sum_{l=1}^{\pi(j)-1} \delta_{\pi^{-1} l} .
$$

4.2.3. Зашнурованные прямоугольники и абелевы дифференциалы. Для заданного зашнурованного прямоугольника $\mathscr{X}=(\lambda, \pi, \delta)$, У. Вич [37] рассматривает $m$ прямоугольников $\Pi_{i}=\Pi_{i}(\lambda, \pi, \delta)$ ширины $\lambda_{i}$ и высоты $h_{i}$, $i=1, \ldots, m$, и склеивает их по правилам, определяемым по перестановке $\pi$. В результате мы получаем риманову поверхность $M=M(\mathscr{X})$ с голоморфной 1 -формой $\omega=\omega(\mathscr{X})$, которая в ограничении на каждый из прямоугольников $\Pi_{i}$ есть просто $d z=d x+i d y$. Объединение оснований прямоугольников является интервалом $I^{(0)}(\lambda, \pi, \delta)$ длины $|\lambda|$ на $M$; отображение первого возвращения на этот интервал для вертикального потока формы $\omega$ есть в точности перекладывание отрезков $\mathbf{T}_{(\lambda, \pi)}$.

Площадь зашнурованного прямоугольника $(\lambda, \pi, \delta)$ задается формулой

$$
\operatorname{Area}(\lambda, \pi, \delta):=\sum_{r=1}^{m} \lambda_{r} h_{r}=\sum_{r=1}^{m} \lambda_{r}\left(-\sum_{i=1}^{r-1} \delta_{i}+\sum_{i=1}^{\pi r-1} \delta_{\pi^{-1} i}\right) .
$$

(Мы считаем, что $\sum_{i=u}^{v} \ldots=0$, если $u>v$.)

4.2.4. Пространство зашнурованных прямоугольников. Обозначим $\mathscr{V}(\mathscr{R})$ пространство зашнурованных прямоугольников, отвечающее классу Рози $\mathscr{R}$, т. е.

$$
\mathscr{V}(\mathscr{R})=\left\{\mathscr{X}=(\lambda, \pi, \delta): \lambda \in \mathbb{R}_{+}^{m}, \pi \in \mathscr{R}, \delta \in K(\pi)\right\},
$$


а $\mathscr{V}_{\text {uе }}(\mathscr{R})$ - его подмножество, состоящее из тех зашнурованных прямоугольников, для которых у отвечающих им абелевых дифференциалов строго эргодичны вертикальный и горизонтальный потоки. Пусть также

$$
\begin{aligned}
& \mathscr{V}^{+}(\mathscr{R})=\left\{(\lambda, \pi, \delta) \in \mathscr{V}(\mathscr{R}): \lambda_{\pi^{-1} m}>\lambda_{m}\right\}, \\
& \mathscr{V}^{-}(\mathscr{R})=\left\{(\lambda, \pi, \delta) \in \mathscr{V}(\mathscr{R}): \lambda_{\pi^{-1} m}<\lambda_{m}\right\}, \\
& \mathscr{V}^{ \pm}(\mathscr{R})=\mathscr{V}^{+}(\mathscr{R}) \cup \mathscr{V}^{-}(\mathscr{R}) .
\end{aligned}
$$

У. Вич [37] вводит поток $\left\{P^{t}\right\}$, действующий на $\mathscr{V}(\mathscr{R})$ по формуле

$$
P^{t}(\lambda, \pi, \delta)=\left(e^{t} \lambda, \pi, e^{-t} \delta\right),
$$

и отображение $\mathscr{U}: \mathscr{V}^{ \pm}(\mathscr{R}) \rightarrow \mathscr{V}(\mathscr{R})$, где

$$
\mathscr{U}(\lambda, \pi, \delta)= \begin{cases}\left(\mathscr{A}(\pi, a)^{-1} \lambda, a \pi, \mathscr{A}(\pi, a)^{-1} \delta\right), & \text { если } \lambda_{\pi^{-1} m}>\lambda_{m} \\ \left(\mathscr{A}(\pi, b)^{-1} \lambda, b \pi, \mathscr{A}(\pi, b)^{-1} \delta\right), & \text { если } \lambda_{\pi^{-1} m}<\lambda_{m} .\end{cases}
$$

(Включение $\mathscr{U} \mathscr{V}^{ \pm}(\mathscr{R}) \subset \mathscr{V}(\mathscr{R})$ доказано в [37].) Отображение $\mathscr{U}$ и поток $\left\{P^{t}\right\}$ коммутируют на $\mathscr{V}^{ \pm}(\mathscr{R})$ и сохраняют меру, определяемую на $\mathscr{V}(\mathscr{R})$ формой объема $\mathrm{Vol}=d \lambda_{1} \cdots d \lambda_{m} d \delta_{1} \cdots d \delta_{m}$. Они также сохраняют площадь зашнурованного прямоугольника (см. (36)), и поэтому их можно ограничить на множество

$$
\mathscr{V}^{1, \pm}(\mathscr{R}):=\left\{(\lambda, \pi, \delta) \in \mathscr{V}^{ \pm}(\mathscr{R}): \operatorname{Area}(\lambda, \pi, \delta)=1\right\} .
$$

Ограничение формы объема Vol на множество $\mathscr{V}^{1, \pm}(\mathscr{R})$ задает на нем меру $\mu_{\mathscr{R}}$, инвариантную относительно действия $\mathscr{U}$ и $\left\{P^{t}\right\}$.

Обозначим для $(\lambda, \pi) \in \Delta(\mathscr{R})$

$$
\tau^{0}(\lambda, \pi)=:-\log \left(|\lambda|-\min \left(\lambda_{m}, \lambda_{\pi^{-1} m}\right)\right) .
$$

Из (32), (33) следует, что если $\lambda \in \Delta_{\pi}^{+} \cup \Delta_{\pi}^{-}$, то

$$
\tau^{0}(\lambda, \pi)=-\log \left|\mathscr{A}^{-1}(\lambda, \pi) \lambda\right| .
$$

Далее, обозначим

$$
\begin{aligned}
\mathscr{Y}_{1}(\mathscr{R}) & :=\{\mathscr{X}=(\lambda, \pi, \delta) \in \mathscr{V}(\mathscr{R}):|\lambda|=1, \operatorname{Area}(\lambda, \pi, \delta)=1\}, \\
\tau(\mathscr{X}) & :=\tau^{0}(\lambda, \pi) \quad \text { для } \mathscr{X}=(\lambda, \pi, \delta) \in \mathscr{Y}_{1}(\mathscr{R}), \\
\mathscr{V}_{1, \tau}(\mathscr{R}) & :=\bigcup_{\mathscr{X} \in \mathscr{Y}_{1}(\mathscr{R}), 0 \leqslant t \leqslant \tau(\mathscr{X})} P^{t} \mathscr{X} .
\end{aligned}
$$

Пусть

$$
\begin{aligned}
\mathscr{V}_{\neq}^{1, \pm}(\mathscr{R}) & :=\left\{(\lambda, \pi, \delta) \in \mathscr{V}^{1, \pm}(\mathscr{R}): a_{m}(\delta) \neq 0\right\}, \\
\mathscr{V}_{\infty}(\mathscr{R}) & :=\bigcap_{n \in \mathbb{Z}} \mathscr{U}^{n} \mathscr{V}_{\neq}^{1, \pm}(\mathscr{R}) .
\end{aligned}
$$

Очевидно, что $\mathscr{U}^{n}$ корректно определено на $\mathscr{V}_{\infty}(\mathscr{R})$ при всех $n \in \mathbb{Z}$. 
Положим теперь

$$
\mathscr{Y}^{\prime}(\mathscr{R}):=\mathscr{Y}_{1}(\mathscr{R}) \cap \mathscr{V}_{\infty}(\mathscr{R}), \quad \widetilde{\mathscr{V}}(\mathscr{R}):=\mathscr{V}_{1, \tau}(\mathscr{R}) \cap \mathscr{V}_{\infty}(\mathscr{R})
$$

Неформально говоря, $\widetilde{\mathscr{V}}(\mathscr{R})$ является "фундаментальной областью" отображения $\mathscr{U}$. Таким образом, на множестве $\widetilde{\mathscr{V}}(\mathscr{R})$ корректно определен поток $\left\{P^{t}\right\}$, для которого мы сохраняем то же обозначение. (Хотя ограниченная положительная функция $\tau$ не отделена от нуля, определение потока $\left\{P^{t}\right\}$ корректно.)

Заметим, что для любого $s \in \mathbb{R}$ имеется естественное “тавтологическое" отображение

$$
\mathfrak{t}_{s}: M(\mathscr{X}) \rightarrow M\left(P^{s} \mathscr{X}\right),
$$

которое на каждом прямоугольнике $\Pi_{i}$ является просто растяжением в $e^{s}$ раз в горизонтальном направлении и сжатием в $e^{s}$ раз в вертикальном. По определению, отображение $\mathfrak{t}_{s}$ переводит вертикальное и горизонтальное слоения на $\mathscr{X}$ в соответствующие слоения на $P^{s} \mathscr{X}$.

Определим пространство

$$
\mathfrak{X} \widetilde{\mathscr{V}}(\mathscr{R})=\{(\mathscr{X}, x): \mathscr{X} \in \widetilde{\mathscr{V}}(\mathscr{R}), x \in M(\mathscr{X})\}
$$

и введем на нем поток $P^{s, \mathfrak{X}}$ по формуле

$$
P^{s, \mathfrak{X}}(\mathscr{X}, x)=\left(P^{s} \mathscr{X}, \mathfrak{t}_{s} x\right) .
$$

Поток $P^{s}$ индуцирует на трансверсали $\mathscr{Y}^{\prime}(\mathscr{R})$ отображение первого возвращения $\overline{\mathscr{T}}$ по формуле

$$
\overline{\mathscr{T}}(\lambda, \pi, \delta)=\mathscr{U} P^{\tau^{0}(\lambda, \pi)}(\lambda, \pi, \delta) .
$$

Заметим, что, по определению, если $\bar{T}(\lambda, \pi, \delta)=\left(\lambda^{\prime}, \pi^{\prime}, \delta^{\prime}\right)$, то $\left(\lambda^{\prime}, \pi^{\prime}\right)=$ $\mathscr{T}(\lambda, \pi)$.

Для $(\lambda, \pi, \delta) \in \widetilde{\mathscr{V}}(\mathscr{R}), s \in \mathbb{R}$, определим $\widetilde{n}(\lambda, \pi, \delta, s)$ формулой

$$
\mathscr{U}^{\widetilde{n}(\lambda, \pi, \delta, s)}\left(e^{s} \lambda, \pi, e^{-s} \delta\right) \in \mathscr{V}_{1, \tau}(\mathscr{R}) .
$$

Зададим на пространстве $\widetilde{\mathscr{V}}(\mathscr{R})$ матричный коцикл $\overline{\mathscr{A}}^{t}$ над потоком $P^{s}$ формулой

$$
\overline{\mathscr{A}}^{t}(s,(\lambda, \pi, \delta))=\mathscr{A}^{t}(\widetilde{n}(\lambda, \pi, \delta, s),(\lambda, \pi)) .
$$

4.2.5. Соответствие между коциклами. Напомним определение коцикла Концевича-Зорича.

Пусть $k=\left(k_{1}, \ldots, k_{\sigma}\right)$ - неотрицательный целочисленный вектор, для которого $k_{1}+\cdots+k_{\sigma}=2 \rho-2$. Обозначим $\mathscr{M}_{\kappa}$ пространство модулей абелевых дифференциалов $(M, \omega)$, где $M$ - риманова поверхность рода $\rho$, а $\omega$ - голоморфный дифференциал площади 1 с особыми точками порядков $k_{1}, \ldots, k_{\sigma}$. Пространство $\mathscr{M}_{\kappa}$ часто называют стратом в пространстве модулей абелевых дифференциалов.

Поток Тейхмюллера $\mathbf{g}_{s}$ на $\mathscr{M}_{\kappa}$ переводит модуль пары $(M, \omega)$ в модуль пары $\left(M, \omega^{\prime}\right)$, где $\omega^{\prime}=e^{s} \operatorname{Re} \omega+i e^{-s} \operatorname{Im} \omega ;$ новая комплексная структура на $M$ 
однозначно определяется требованием голоморфности формы $\omega^{\prime}$. Как показал Вич, пространство $\mathscr{M}_{\kappa}$ может быть несвязным; пусть $\mathscr{H}$ - компонента связности $\mathscr{M}_{\kappa}$.

Пусть $\mathbb{H}^{1}(\mathscr{H})$ - расслоение над $\mathscr{H}$, слой которого над точкой $(M, \omega)$ - это группа когомологий $H^{1}(M, \mathbb{R})$. На расслоении $\mathbb{H}^{1}(\mathscr{H})$ задана связность Гаусса-Манина, для которой все непрерывные целочисленные сечения этого расслоения являются плоскими; связность задается этим условием однозначно. Параллельный перенос относительно связности Гаусса-Манина вдоль орбит потока Тейхмюллера порождает коцикл над потоком Тейхмюллера, называемый кочиклом Концевича-Зорича $\mathbf{A}_{\mathrm{KZ}}$ [29], [30].

Коцикл Концевича-Зорича $\mathbf{A}_{\mathrm{KZ}}$ порождает поток $g_{s}^{\mathbf{A}_{\mathrm{KZ}}}$ на $\mathbb{H}^{1}(\mathscr{H})$, имеющий вид косого произведения и задаваемый формулой

$$
g_{s}^{\mathbf{A}_{\mathrm{KZ}}}(\mathbf{X}, v)=\left(\mathbf{g}_{s} \mathbf{X}, \mathbf{A}_{\mathrm{KZ}} v\right), \quad \mathbf{X} \in \mathscr{H}, \quad v \in H^{1}(M, \mathbb{R}) .
$$

Каждой связной компоненте $\mathscr{H}$ пространства $\mathscr{M}_{\kappa}$ можно поставить в соответствие класс Рози $\mathscr{R}$, для которого верно следующее [30], [37], [40].

Теорема 3 (У. Вич). Существует измеримое отображение

$$
\pi_{\mathscr{R}}: \widetilde{V}(\mathscr{R}) \rightarrow \mathscr{H}
$$

при котором каждая точка имеет лишь конечное число прообразов, такое, что $\boldsymbol{\pi}_{\mathscr{R}} \circ P^{t}=g_{t} \circ \boldsymbol{\pi}_{\mathscr{R}}$. Образ $\boldsymbol{\pi}_{\mathscr{R}}$ содержит все абелевы дифберенциалы, вертикальные и горизонтальные слоения которых оба минимальны.

Следуя Вичу [37], [41], опишем теперь связь между коциклом Концевича-Зорича $\mathbf{A}_{\mathrm{KZ}}$ и коциклом $\overline{\mathscr{A}}^{t}$.

Для любой неприводимой перестановки $\pi$ Вич [38] определяет кососимметричную матрицу $L^{\pi}$, полагая

$$
L_{i j}^{\pi}= \begin{cases}0, & \text { если } i=j \text { или } i<j, \pi i<\pi j ; \\ 1, & \text { если } i<j, \pi i>\pi j \\ -1, & \text { если } i>j, \pi i<\pi j,\end{cases}
$$

и обозначает символом $N(\pi)$ ядро $L^{\pi}$, а символом $H(\pi)=L^{\pi}\left(\mathbb{R}^{m}\right)$ - образ $L^{\pi}$. Размерности пространств $N(\pi)$ и $H(\pi)$ одинаковы для всех перестановок $\pi$ из $\mathscr{R}$, и, более того, как установил Вич [38], имеют место следующие свойства пространств $N(\pi), H(\pi)$.

ПрЕДЛОЖЕНИЕ 4.3. Пустъ $c=a$ или $b$. Тогда:

1) $H(c \pi)=\mathscr{A}^{t}(c, \pi) H(\pi), N(c \pi)=\mathscr{A}^{-1}(c, \pi) N(\pi)$;

2) диаграмма

$$
\begin{array}{cc}
\mathbb{R}^{m} / N(\pi) \stackrel{L^{\pi}}{\longrightarrow} & H(\pi) \\
\downarrow_{\mathscr{A}^{-1}(c, \pi)} & \downarrow^{t}(c, \pi) \\
\mathbb{R}^{m} / N(c \pi) \stackrel{L^{c \pi}}{\longrightarrow} & H(c \pi)
\end{array}
$$

коммутативна и каждая стрелка в ней - изоморфизм; 
3) для каждой перестановки $\pi$ существует такой базис $\mathbf{v}_{\pi}$ в $N(\pi)$, что отображение $\mathscr{A}^{-1}(c, \pi)$ переводит каждый элемент $\mathbf{v}_{\pi}$ в элемент $\mathbf{v}_{c \pi}$.

Тем самым, на каждом пространстве $H^{\pi}$ имеется естественная кососимметричная билинейная форма $\mathscr{L}_{\pi}$, определяемая для $v_{1}, v_{2} \in H(\pi)$ как

$$
\mathscr{L}_{\pi}\left(v_{1}, v_{2}\right)=\left\langle v_{1},\left(L^{\pi}\right)^{-1} v_{2}\right\rangle
$$

(Вектор $\left(L^{\pi}\right)^{-1} v_{2}$ лежит в $\mathbb{R}^{m} / N(\pi)$; поскольку при всех $v_{1} \in H(\pi), v_{2} \in N(\pi)$ по определению $\left\langle v_{1}, v_{2}\right\rangle=0$, правая часть корректно определена.)

Рассмотрим $\mathscr{T}^{\mathscr{A}^{t}}$-инвариантное подрасслоение $\mathscr{H}(\Delta(\mathscr{R})) \subset \Delta(\mathscr{R}) \times \mathbb{R}^{m}$, задаваемое формулой

$$
\mathscr{H}(\Delta(\mathscr{R}))=\{((\lambda, \pi), v):(\lambda, \pi) \in \Delta(\mathscr{R}), v \in H(\pi)\},
$$

а также фактор-расслоение

$$
\mathscr{N}(\Delta(\mathscr{R}))=\left\{((\lambda, \pi), v):(\lambda, \pi) \in \Delta(\mathscr{R}), v \in \mathbb{R}^{m} / N(\pi)\right\} .
$$

Отображение расслоений

$$
\mathscr{L}_{\mathscr{R}}: \mathscr{H}(\Delta(\mathscr{R})) \rightarrow \mathscr{N}(\Delta(\mathscr{R})), \quad \mathscr{L}_{\mathscr{R}}((\lambda, \pi), v)=\left((\lambda, \pi), L^{\pi} v\right),
$$

индуцирует изоморфизм расслоений между $\mathscr{H}(\Delta(\mathscr{R}))$ и $\mathscr{N}(\Delta(\mathscr{R}))$.

Оба расслоения естественным образом поднимаются до расслоений $\mathscr{H}(\widetilde{\mathcal{V}}(\mathscr{R}))$, $\mathscr{N}(\widetilde{\mathscr{V}}(\mathscr{R}))$ над пространством $\widetilde{\mathscr{V}}(\mathscr{R})$ зашнурованных прямоугольников; они естественно инвариантны относительно соответствующих потоков косых произведений $P^{s, \overline{\mathscr{A}}^{t}}, P^{s, \overline{\mathscr{A}}^{-1}}$, и отображение $\mathscr{L}_{\mathscr{R}}$ поднимается до изоморфизма расслоений между $\mathscr{H}(\widetilde{\mathscr{V}}(\mathscr{R}))$ и $\mathscr{N}(\widetilde{\mathcal{V}}(\mathscr{R}))$.

Возьмем $\mathscr{X} \in \widetilde{\mathscr{V}}(\mathscr{R})$ и положим $\boldsymbol{\pi}_{\mathscr{R}}(\mathscr{X})=(M(\mathscr{X}), \omega(\mathscr{X}))$. Вич [39] показал, что отображение $\boldsymbol{\pi}_{\mathscr{R}}$ поднимается до эпиморфизма расслоений $\widetilde{\boldsymbol{\pi}}_{\mathscr{R}}$ из $\mathscr{H}(\widetilde{\mathscr{V}}(\mathscr{R}))$ на $\mathbb{H}^{1}(\mathscr{H})$, который сплетает коцикл $\overline{\mathscr{A}}^{t}$ и коцикл Концевича-Зорича $\mathbf{A}_{\mathrm{KZ}}$.

ПреДЛОЖение 4.4 (У. Вич). Для почти всех $\mathscr{X} \in \widetilde{\mathscr{V}}(\mathscr{R}), \mathscr{X}=(\lambda, \pi, \delta)$, существует такой изоморфизм $\mathscr{I}_{\mathscr{X}}: H(\pi) \rightarrow H^{1}(M(\mathscr{X}), \mathbb{R})$, что

1) отображение $\widetilde{\boldsymbol{\pi}}_{\mathscr{R}}: \mathscr{H}(\Delta(\mathscr{R})) \rightarrow \mathbb{H}^{1}(\mathscr{H})$, заданное формулой

$$
\tilde{\boldsymbol{\pi}}_{\mathscr{R}}(\mathscr{X}, v)=\left(\boldsymbol{\pi}_{\mathscr{R}}(\mathscr{X}), \mathscr{I}_{\mathscr{X}} v\right),
$$

индуцирует эпиморфизм измеримых расслоений из $\mathscr{H}(\Delta(\mathscr{R}))$ на $\mathbb{H}^{1}(\mathscr{H})$;

2) диаграмма

$$
\begin{array}{cc}
\mathscr{H}(\widetilde{\mathscr{V}}(\mathscr{R})) \stackrel{\tilde{\pi}_{\mathscr{R}}}{\longrightarrow} & \mathbb{H}^{1}(\mathscr{H}) \\
\downarrow_{P^{s, \overline{\mathscr{A}}^{t}}} & \downarrow g_{s}^{\mathbf{A}_{\mathrm{KZ}}} \\
\mathscr{H}(\widetilde{\mathscr{V}}(\mathscr{R})) \stackrel{\tilde{\boldsymbol{\pi}}_{\mathscr{R}}}{\longrightarrow} & \mathbb{H}^{1}(\mathscr{H})
\end{array}
$$

коммутативна;

3) если $\mathscr{X}=(\lambda, \pi, \delta)$, то изоморфизм $\mathscr{I}_{X}$ переводит билинейную форму $\mathscr{L}_{\pi}$ на $H(\pi)$, определенную в $(39)$, в $\cup$-произведение в $H^{1}(M(\mathscr{X}), \mathbb{R})$. 
ДокАзАтельство. Каждому прямоугольнику $\Pi_{i}$ У. Вич [38] сопоставляет цикл $\gamma_{i}(\lambda, \pi, \delta)$ в группе гомологий $H_{1}(M, \mathbb{Z})$ : если $P_{i}-$ левый нижний угол $\Pi_{i}$, a $Q_{i}$ - его левый верхний угол, то цикл представляет собой объединение вертикального отрезка $P_{i} Q_{i}$ и горизонтального подотрезка в $I^{(0)}(\lambda, \pi, \delta)$, соединяющего $Q_{i}$ и $P_{i}$. Ясно, что циклы $\gamma_{i}(\lambda, \pi, \delta)$ порождают пространство $H_{1}(M, \mathbb{Z})$; более того, Вич доказал, что цикл $t_{1} \gamma_{1}+\cdots+t_{m} \gamma_{m}$ гомологичен нулю тогда и только тогда, когда $\left(t_{1}, \ldots, t_{m}\right) \in N(\pi)$. Таким образом, мы получаем отождествление $\mathbb{R}^{m} / N(\pi)$ и $H_{1}(M, \mathbb{R})$. Аналогично, подпространство в $\mathbb{R}^{m}$, натянутое на векторы $\left(f\left(\gamma_{1}\right), \ldots, f\left(\gamma_{m}\right)\right), f \in H^{1}(M, \mathbb{R})$, - это в точности $H(\pi)$. Отождествление билинейной формы $\mathscr{L}_{\pi}$ и $\cup$-произведения устанавливается в предложении 4.19 в [42]. Предложение доказано.

Третье утверждение предложения 4.3 имеет следующее важное следствие.

СледСтвиЕ 4.5. Пусть $\mathrm{P}_{\mathscr{V}}-$ эргодическая $\mathrm{P}^{s}$-инвариантная вероятностная мера для потока $P^{s}$ на $\mathscr{V}(\mathscr{R})$, а $\mathrm{P}_{\mathscr{H}}=\left(\boldsymbol{\pi}_{\mathscr{R}}\right)_{*} \mathrm{P}_{\mathscr{V}}-$ соответствующая

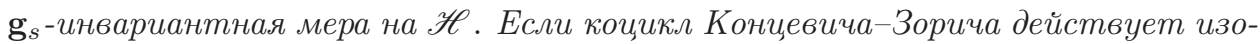
метрично на своем нейтральном подпространстве по отношению к мере $\mathrm{P}_{\mathscr{H}}$, то коцикл $\overline{\mathscr{A}}^{t}$ также действует изометрично на своем нейтральном подпространстве относительно $\mathrm{P}_{\mathscr{V}}$.

Отметим, что предположения следствия 4.5 выполняются, в частности, для гладкой меры Мазура-Вича на пространстве модулей абелевых дифференциалов [33], [37].

ЗАмечАниЕ. О ляпуновском спектре в других случаях см., например, [17].

\section{3. Зашнурованные прямоугольники и марковские компакты}

4.3.1. Основные леммы. Перейдем к детальному изложению связи между зашнурованными прямоугольниками Вича и марковскими компактами.

Для любого конечного множества $\mathfrak{G}_{0} \subset \mathfrak{G}$ обозначим

$$
\Omega_{\mathfrak{G}_{0}}=\left\{\omega \in \Omega: \omega_{n} \in \mathfrak{G}_{0}, n \in \mathbb{Z}\right\}, \quad \bar{\Omega}_{\mathfrak{G}_{0}}=\left\{\bar{\omega}=(\omega, r): \omega \in \Omega_{\mathfrak{G}_{0}}\right\} .
$$

Наша первая лемма предъявляет отображение из пространства Вича зашнурованных прямоугольников в пространство $\bar{\Omega}$ измеримых марковских компактов.

Лемма 4.6. Пусть $\mathscr{R}$ - некоторый класс Рози неприводимых перестановок. Существуют конечное множество $\mathfrak{G} \mathscr{R} \subset \mathfrak{G}$, а также упорядочения Вершика о и обратные упорядочения Вершика $\widetilde{\mathfrak{o}}$ на каждом из графов $\Gamma \in \mathfrak{G}_{\mathscr{R}}$, для которых верно следующее. Существует отображение

$$
\overline{\mathfrak{Z}}_{\mathscr{R}}: \mathscr{V}_{u e}(\mathscr{R}) \rightarrow \bar{\Omega}_{\mathfrak{G}_{\mathscr{R}}},
$$


для которого диаграмма

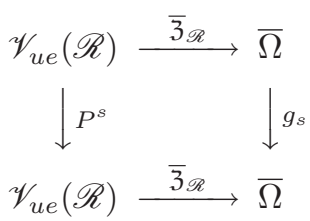

коммутативна, и если $\mathrm{P}_{\mathscr{V}}$ - эргодическая вероятностная $P^{s}$-инвариантная мера на $\mathscr{V}(\mathscr{R})$, то

$$
\left(\overline{\mathfrak{Z}}_{\mathscr{R}}\right)_{*} \mathrm{P}_{\mathscr{V}} \in \mathscr{P}^{+} .
$$

Вторая лемма устанавливает соответствие между зашнурованным прямоугольником $\mathscr{X}$ и соответствующим марковским компактом $\overline{\mathfrak{Z}}_{\mathscr{R}}(\mathscr{X})$.

Лемма 4.7. Для любого $\mathscr{X} \in \mathscr{V}_{\text {ие}}(\mathscr{R})$ существует такое определенное почти всюду по мере Лебега отображение

$$
\overline{\mathscr{J}}_{\mathscr{X}}: M(\mathscr{X}) \rightarrow X\left(\bar{\omega}_{\mathscr{X}}\right),
$$

что отображение

$$
\overline{\mathfrak{Z}}_{\mathscr{R}}^{\mathfrak{X}}: \mathfrak{X} \mathscr{V}_{u e}(\mathscr{R}) \rightarrow \mathfrak{X} \bar{\Omega},
$$

задаваемое формулой

$$
\overline{\mathfrak{Z}}_{\mathscr{R}}^{\mathfrak{X}}(\mathscr{X}, x)=\left(\overline{\mathfrak{Z}}_{\mathscr{R}} \mathscr{X}, \overline{\mathscr{J}}_{\mathscr{X}} x\right),
$$

делает коммутативной диаграмму

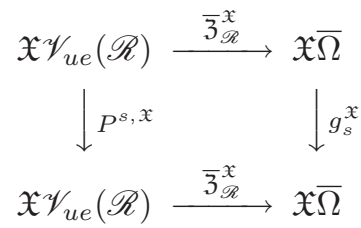

Отображение $\overline{\mathscr{J}}_{\mathscr{X}}$ переводит вертикальный поток $h_{t}^{+}$на $\mathscr{X}$ в поток $h_{t}^{+, \mathfrak{o}}$ на $X\left(\overline{\mathfrak{Z}}_{\mathscr{R}}(\mathscr{X})\right)$, горизонтальный поток $h_{t}^{-}$на $\mathscr{X}$ в поток $h_{t}^{-, \widetilde{o}}$ на $X\left(\overline{\mathfrak{Z}}_{\mathscr{R}}(\mathscr{X})\right)$ и индуиирует изоморфизмы между пространством $\mathfrak{B}_{\mathscr{X}}^{+}$и пространством $\mathfrak{B}_{\overline{\mathfrak{Z}}_{\mathscr{R}}(\mathscr{X})}$ и между пространством $\mathfrak{B}_{\mathscr{X}}^{-}$и пространством $\mathfrak{B}_{\overline{\mathfrak{Z}}_{\mathscr{R}}}^{-}(\mathscr{X}) \cdot$

Третья лемма показывает, что отображение $\overline{\mathfrak{Z}}_{\mathscr{R}}$ сплетает коцикл Концевича-Зорича и ренормализационный коцикл.

Лемма 4.8. Если отображение

$$
\overline{\mathfrak{Z}}_{\mathscr{R}}^{\prime}: \mathscr{V}_{u e}(\mathscr{R}) \times \mathbb{R}^{m} \rightarrow \bar{\Omega} \times \mathbb{R}^{m}
$$

задано формулой

$$
\overline{\mathfrak{Z}}_{\mathscr{R}}^{\prime}(\mathscr{X}, v)=\left(\overline{\mathfrak{Z}}_{\mathscr{R}} \mathscr{X}, v\right),
$$


то диаграмма

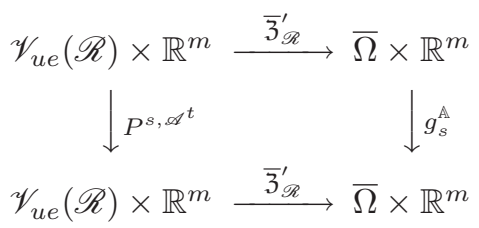

коммутативна.

Неформально говоря, отображение $\overline{\mathfrak{Z}}_{\mathscr{R}}$ строится следующим образом. Сначала каждому зашнурованному прямоугольнику ставится в соответствие его код Рози-Вича, т. е. бесконечная в обе стороны последовательность пар $(\pi, c)$, $\pi \in \mathscr{R}, c=a$ или $b$. Каждой паре $(\pi, c)$ сопоставляется унимодулярная матрица $\mathscr{A}(c, \pi)$. Наконец, каждой матрице сопоставляется граф Г из $\mathfrak{G}$. Полученная последовательность графов и задает искомый марковский компакт.

ЗАмЕчаниЕ. В [43] Вич доказал, что если перекладывание отрезков $(\lambda, \pi)$ минимально, то последовательность его ренормализационных матриц РозиВича $\mathscr{A}\left(\mathscr{T}^{n}(\lambda, \pi)\right)$ однозначно задает перестановку $\pi$. В частности, если $(\lambda, \pi)$ строго эргодично, то последовательность ренормализационных матриц РозиВича однозначно задает такое перекладывание. Из этого утверждения Вича вытекает, что отображение $\overline{\mathfrak{Z}}_{\mathscr{R}}$ в действительности инбективно.

4.3.2. Коды Рози-Вича зашнурованных прямоугольников. Если задан класс Рози $\mathscr{R}$ неприводимых перестановок, рассмотрим алфавит

$$
\mathfrak{A}_{\mathscr{R}}=\{(\pi, c): c=a \text { или } b, \pi \in \mathscr{R}\} .
$$

Каждой букве $\mathbf{p} \in \mathfrak{A}_{\mathscr{R}}$ поставим в соответствие множество $\Delta_{\mathbf{p}} \subset \Delta(\mathscr{R})$ по формуле

$$
\Delta_{\mathbf{p}}= \begin{cases}\Delta_{\pi}^{+}, & \text {если } \mathbf{p}=(\pi, a), \\ \Delta_{\pi}^{-}, & \text {если } \mathbf{p}=(\pi, b) .\end{cases}
$$

Рассмотрим зашнурованный прямоугольник $\mathscr{X} \in \mathscr{Y}^{\prime}(\mathscr{R}), \mathscr{X}=(\lambda, \pi, \delta)$, $|\lambda|=1$. При $n \in \mathbb{Z}$ будем обозначать $\mathscr{T} \mathscr{X}=\left(\lambda^{(n)}, \pi^{(n)}, \delta^{(n)}\right)$. Поставим в соответствие зашнурованному прямоугольнику $\mathscr{X}$ последовательность $\left(\mathbf{p}_{n}(\mathscr{X})\right)_{n \in \mathbb{Z}}$ такую, что

$$
\left(\lambda^{(n)}, \pi^{(n)}\right) \in \Delta_{\mathbf{p}_{n}}
$$

Последовательность $\left(\mathbf{p}_{n}(\mathscr{X})\right)_{n \in \mathbb{Z}}$ - это код Рози-Вича зашнурованного прямоугольника $\mathscr{X}$.

4.3.3. Марковский компакт, соответствующий зашнурованному прямоугольнику. Поставим в соответствие каждой букве $\mathbf{p} \in \mathfrak{A}_{\mathscr{R}}$ следующий ориентированный граф $\Gamma(\mathbf{p})$ с $m$ вершинами. 
СлучАй 1. Пусть $\mathbf{p}=(\pi, a)$. Тогда граф $\Gamma(\mathbf{p})$ состоит из $m+1$ ребра:

$$
\begin{aligned}
& e_{i i}, \quad 1 \leqslant i \leqslant \pi^{-1} m, \\
& e_{\pi^{-1} m+1, m}, \\
& e_{i, i-1}, \quad \pi^{-1} m+1 \leqslant i \leqslant m,
\end{aligned}
$$

где $I\left(e_{i j}\right)=i, F\left(e_{i j}\right)=j$.

СлУчАй 2. Пусть $\mathbf{p}=(\pi, b)$. Тогда граф $\Gamma(\mathbf{p})$ состоит из $m+1$ ребра:

$$
e_{i i}, i=1, \ldots, m, \quad e_{\pi^{-1} m, m},
$$

где мы снова полагаем $I\left(e_{i j}\right)=i, F\left(e_{i j}\right)=j$.

Матрица инцидентности графа $\Gamma(\mathbf{p}), \mathbf{p}=(\pi, c)$, тем самым, является транспонированной матрицей Рози, отвечающей $(\pi, c)$.

Каноническое упорядочение Вершика о на графах $Г(\mathbf{p})$ зададим следующим образом: $e_{i j}<e_{i k}$ тогда и только тогда, когда $j<k$, а обратное упорядочение Вершика $\widetilde{\mathfrak{o}}$ зададим так: $e_{j i}<e_{k i}$ тогда и только тогда, когда $j<k$.

Слово $\left(\mathbf{p}_{1}, \ldots, \mathbf{p}_{l}\right), \mathbf{p}_{i}=\left(\pi_{i}, c_{i}\right) \in \mathfrak{A}_{\mathscr{R}}$, будем называть допустимым, если $\pi_{i+1}=c_{i} \pi_{i}$. Аналогично, бесконечную последовательность букв будем называть допустимой, если допустима любая ее конечная подпоследовательность. Множество допустимых двусторонних последовательностей обозначим $\Sigma \mathscr{R}$, а односторонних $-\Sigma_{\mathscr{R}}^{+}$. Имеется естественное отображение $\operatorname{Gr}_{\mathscr{R}}: \Sigma_{\mathscr{R}} \rightarrow \Omega$, заданное формулой

$$
\operatorname{Gr}_{\mathscr{R}}:\left(\mathbf{p}_{n}\right)_{n \in \mathbb{Z}} \mapsto\left(\Gamma\left(\mathbf{p}_{n}\right)\right)_{n \in \mathbb{Z}} .
$$

Имеется также естественное отображение $\operatorname{Code}_{\mathscr{R}}^{+}: \Delta(\mathscr{R}) \rightarrow \Sigma_{\mathscr{R}}^{+}$, которое переводит $(\lambda, \pi)$ в последовательность $\left(\mathbf{p}_{n}\right)_{n \in \mathbb{N}}$ такую, что

$$
\mathscr{T}^{n}(\lambda, \pi) \in \Delta_{\mathbf{p}_{n}} .
$$

Это отображение расширяется до отображения $\operatorname{Code}_{\mathscr{R}}: \mathscr{Y}^{\prime}(\mathscr{R}) \rightarrow \Sigma_{\mathscr{R}}$, сопоставляющего $(\lambda, \pi, \delta)$ последовательность $\left(\mathbf{p}_{n}\right)_{n \in \mathbb{Z}}$, где

$$
\overline{\mathscr{T}}^{n}(\lambda, \pi, \delta)=\left(\lambda^{(n)}, \pi^{(n)}, \delta^{(n)}\right), \quad\left(\lambda^{(n)}, \pi^{(n)}\right) \in \Delta_{\mathbf{p}_{n}} .
$$

Обозначим

$$
\mathfrak{Z}_{\mathscr{R}}=\operatorname{Gr}_{\mathscr{R}} \circ \operatorname{Code}_{\mathscr{R}}: \mathscr{Y}^{\prime}(\mathscr{R}) \rightarrow \Omega .
$$

4.3.4. Свойства символического кодирования. Итак, мы построили измеримое символическое кодирование

$$
\mathfrak{Z}_{\mathscr{R}}: \mathscr{Y}^{\prime}(\mathscr{R}) \rightarrow \Omega,
$$

и диаграмма

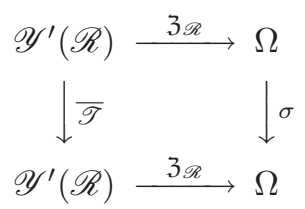

коммутативна по построению. 
Рассмотрим для зашнурованного прямоугольника $\mathscr{X} \in \mathscr{Y}^{\prime}(\mathscr{R})$ соответствующий абелев дифференциал $\mathbf{X}=\boldsymbol{\pi}_{\mathscr{R}}(\mathscr{X})$ на поверхности $M(\mathscr{X})$, и пусть $\mathbf{m}_{\mathscr{X}}-$ мера Лебега на $M(\mathscr{X})$. Положим $\omega_{\mathscr{X}}=\mathfrak{Z}_{\mathscr{R}}(\mathscr{X})$. Тогда имеется "тавтологическое" кодирующее отображение из марковского компакта $X\left(\omega_{\mathscr{X}}\right)$ в $M(\mathscr{X})$. Слоения $\mathscr{F}_{X\left(\omega_{\mathscr{X}}\right)}^{+}$и $\mathscr{F}_{X\left(\omega_{\mathscr{X}}\right)}^{-}$являются соответственно вертикальным и горизонтальным слоениями на $M(\mathscr{X})$; строгая эргодичность марковского компакта $X\left(\omega_{\mathscr{X}}\right)$ равносильна строгой эргодичности горизонтального и вертикального потоков на $M(\mathscr{X})$.

Предположим теперь, что марковский компакт $X\left(\omega_{\mathscr{X}}\right)$ строго эргодичен. Тогда кодирующее отображение обратимо $\nu_{\omega_{\mathscr{X}}}$-почти наверное, и возникает m $\mathscr{X}$-почти наверное определенное отображение

$$
\mathscr{J}_{\mathscr{X}}: M(\mathscr{X}) \rightarrow X\left(\omega_{\mathscr{X}}\right)
$$

По определению, отображение $\mathscr{J} \mathscr{X}$ индуцирует линейный изоморфизм между пространствами $\mathfrak{B}_{\mathbf{X}}^{+}$и $\mathfrak{B}_{X\left(\omega_{\mathscr{X}}\right)}^{+}$; и, аналогично, между пространствами $\mathfrak{B}_{\mathbf{X}}^{-}$ и $\mathfrak{B}_{X\left(\omega_{\mathscr{X}}\right)}$. Тогда $\left(\mathscr{J}_{\mathscr{X}}\right)_{*} \mathbf{m}_{\mathscr{X}}=\nu_{\omega_{\mathscr{X}}}$. Отображение $\mathscr{J}_{\mathscr{X}}$ переводит пространство слабо липшицевых функций на $M(\mathscr{X})$ в пространство слабо липшицевых функций на $X\left(\omega_{\mathscr{X}}\right)$.

Отображение $\mathfrak{Z} \mathscr{R}$ поднимается до естественного отображения

$$
\overline{\mathfrak{Z}}_{\mathscr{R}}: \mathscr{V}_{u e}(\mathscr{R}) \rightarrow \bar{\Omega},
$$

и диаграмма

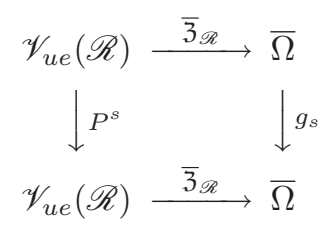

снова коммутативна.

Если $\mathrm{P}_{\mathscr{V}}$ - эргодическая вероятностная $P^{s}$-инвариантная мера, то выполнено включение (40). Действительно, (40) - это переформулировка леммы, принадлежащей Вичу [37], которая утверждает, что любая конечная $P^{s}$-инвариантная мера присваивает положительную вероятность любой матрице Рози с положительными элементами.

Тогда для любого $\mathscr{X} \in \mathscr{V}_{\text {uе }}(\mathscr{R})$ мы снова получаем $\mathbf{m}_{\mathscr{X}}$-почти наверное определенное отображение

$$
\overline{\mathscr{J}}_{\mathscr{X}}: M(\mathscr{X}) \rightarrow X\left(\bar{\omega}_{\mathscr{X}}\right) .
$$

Введем отображение

$$
\overline{\mathfrak{Z}}_{\mathscr{R}}^{\mathfrak{X}}: \mathfrak{X} \mathscr{V}_{u e}(\mathscr{R}) \rightarrow \mathfrak{X} \bar{\Omega}
$$

по формуле

$$
\overline{\mathfrak{Z}}_{\mathscr{R}}^{\mathfrak{X}}(\mathscr{X}, x)=\left(\overline{\mathfrak{Z}}_{\mathscr{R}} \mathscr{X}, \overline{\mathscr{J}}_{\mathscr{X}} x\right) .
$$


Диаграмма

$$
\begin{aligned}
& \mathfrak{X} \mathscr{V}_{\text {ue }}(\mathscr{R}) \stackrel{\overline{\mathfrak{Z}}_{\mathscr{R}}^{\mathfrak{X}}}{\longrightarrow} \\
& \downarrow^{s, \mathfrak{X}} \\
& \mathfrak{X} \mathscr{V}_{\text {ue }}(\mathscr{R}) \stackrel{g^{\mathfrak{X}}}{\stackrel{\overline{\mathfrak{Z}}_{\mathscr{R}}^{\mathfrak{X}}}{\longrightarrow}} \mathfrak{X} \bar{\Omega}
\end{aligned}
$$

коммутативна.

Отображение $\overline{\mathfrak{Z}}_{\mathscr{R}}$ сплетает коциклы $\mathscr{A}^{t}$ и $\mathbb{A}$ в следующем смысле. Для $\mathscr{X} \in$ $\mathscr{V}_{u e}(\mathscr{R}), v \in \mathbb{R}^{m}$ положим

$$
\overline{\mathfrak{Z}}_{\mathscr{R}}^{\prime}(\mathscr{X}, v)=\left(\overline{\mathfrak{Z}}_{\mathscr{R}} \mathscr{X}, v\right)
$$

тогда полученное отображение

$$
\overline{\mathfrak{Z}}_{\mathscr{R}}^{\prime}: \mathscr{V}_{u e}(\mathscr{R}) \times \mathbb{R}^{m} \rightarrow \bar{\Omega} \times \mathbb{R}^{m}
$$

сплетает коциклы $\mathscr{A}^{t}$ и $\mathbb{A}:$ действительно, по определению, диаграмма

$$
\begin{array}{r}
\mathscr{V}_{u e}(\mathscr{R}) \times \mathbb{R}^{m} \stackrel{\overline{\mathfrak{Z}}_{\mathscr{R}}^{\prime}}{\longrightarrow} \bar{\Omega} \times \mathbb{R}^{m} \\
\downarrow_{P^{s, \mathscr{A}^{t}}} \downarrow g_{s}^{\mathbb{A}} \\
\mathscr{V}_{\text {ue }}(\mathscr{R}) \times \mathbb{R}^{m} \stackrel{\overline{\mathfrak{Z}}_{\mathscr{R}}^{\prime}}{\longrightarrow} \bar{\Omega} \times \mathbb{R}^{m}
\end{array}
$$

коммутативна.

Обозначим $\mathfrak{G}_{\mathscr{R}}=\left\{\Gamma(\mathbf{p}), \mathbf{p} \in \mathfrak{A}_{\mathscr{R}}\right\}$ и положим

$$
\Omega_{\mathfrak{G}_{\mathscr{R}}}=\left\{\omega \in \Omega: \omega_{n} \in \mathfrak{G}_{\mathscr{R}}, n \in \mathbb{Z}\right\}, \quad \bar{\Omega}_{\mathfrak{G}_{\mathscr{R}}}=\left\{\bar{\omega}=(\omega, r): \omega \in \Omega_{\mathfrak{G}_{\mathscr{R}}}\right\} .
$$

По построению, $\mathfrak{Z}_{\mathscr{R}}\left(\mathscr{Y}_{\mathscr{R}}^{\prime}\right) \subset \Omega_{\mathfrak{G} \mathscr{R}}$. Каждый граф $\Gamma \in \mathfrak{G}_{\mathscr{R}}$ снабжается упорядочением Вершика как указано в предыдущем п. 4.3.3, и мы получаем $\sigma$-эквивариантное упорядочение Вершика $\mathfrak{o}_{\mathscr{R}}$ на $\mathfrak{Z}_{\mathscr{R}}\left(\mathscr{Y}_{\mathscr{R}}^{\prime}\right)$.

По определению, отображение $\overline{\mathscr{J}} \mathscr{X}$ переводит вертикальный поток $h_{t}^{+}$ на $M(\mathscr{X})$ в поток $h_{t}^{+, \omega \mathscr{X}}$, а горизонтальный поток $h_{t}^{-}$на $M(\mathscr{X})-$ в поток $h_{t}^{-, \omega \mathscr{X}}$.

Леммы 4.6, 4.7 и 4.8 доказаны.

Теоремы 1 и 2 из [14] теперь вытекают, как уже было сказано, из их символических аналогов, следствия 3.4 и теоремы 2 .

ЗАмечАниЕ. Хорошо известно, что коцикл Концевича-Зорича - симплектический. В вышеприведенном доказательстве асимптотики эргодических интегралов эта симплектическая структура нигде не используется. Используется только символическое представление потока Тейхмюллера как ренормализационного потока на пространстве марковских компактов, коцикла Концевича-Зорича как ренормализационного коцикла и то, что коцикл Концевича-Зорича удовлетворяет условиям мультипликативной эргодической теоремы Оселедца. 


\section{Приложение А. О мультипликативной эргодической теореме Оселедца}

А.1. Теорема Оселедца-Песина о редукции. Для удобства читателя мы напомним здесь несколько результатов, уточняющих мультипликативную теорему Оселедца [34]. Первое утверждение, которое нам понадобится, - это немедленное следствие из теоремы Оселедца-Песина о редукции [5]. Мы ограничимся случаем дискретного времени и обратимого отображения, случай потоков совершенно аналогичен.

Пусть $(Y, \mathscr{B}, \mu)$ - вероятностное пространство, а $T: Y \rightarrow Y$ - обратимое сохраняющее меру $\mu$ преобразование. Далее, пусть $m \in \mathbb{N}$ и

$$
\mathbb{A}: Y \rightarrow \mathrm{GL}(m, \mathbb{R})
$$

- измеримое отображение.

Обозначим

$$
\mathbb{A}(n, y)= \begin{cases}\mathbb{A}\left(T^{n-1} y\right) \cdots \mathbb{A}(y), & n>0 \\ \mathrm{Id}, & n=0 \\ \mathbb{A}^{-1}\left(T^{-n} y\right) \cdots \mathbb{A}^{-1}\left(T^{-1} y\right), & n<0\end{cases}
$$

Семейство отображений $\mathbb{A}(n, y), n \in \mathbb{Z}$, называется измеримым коииклом над $T$. Наряду с коциклом $\mathbb{A}$ над автоморфизмом $T$ мы будем рассматривать транспонированный коцикл $\mathbb{A}^{t}$ над автоморфизмом $T^{-1}$, который определяется по формуле

$$
\mathbb{A}^{t}(n, y)= \begin{cases}\mathbb{A}^{t}\left(T^{1-n} y\right) \cdots \mathbb{A}^{t}(y), & n>0 \\ \mathrm{Id}, & n=0 \\ \left(\mathbb{A}^{t}\right)^{-1}\left(T^{-n} y\right) \cdots\left(\mathbb{A}^{t}\right)^{-1}\left(T^{-1} y\right), & n<0\end{cases}
$$

Будем обозначать $\|A\|$ обычную евклидову норму матрицы $A$ и, как и выше, будем для $v \in \mathbb{R}^{m}, v=\left(v_{1}, \ldots, v_{m}\right)$, полагать

$$
|v|=\sum_{i=1}^{m}\left|v_{i}\right| .
$$

Tеорема А.1. Пусть $(Y, \mathscr{B}, \mu)$ - вероятностное пространство, $T: Y \rightarrow Y-$ обратимое эргодическое сохраняющее меру $\mu$ преобразование. Пусть $m \in \mathbb{N}$ u $\mathbb{A}: Y \rightarrow \mathrm{GL}(m, \mathbb{R})$ - измеримое отображение, для которого функции

$$
\log (1+\|A(y)\|) \quad u \quad \log \left(1+\left\|A^{-1}(y)\right\|\right)
$$

принадлежат пространству $L_{1}(Y, \mu)$. Тогда существуют числа $\theta_{1}>\theta_{2}>$ $\cdots>\theta_{r} u$, для $\mu$-почти всех $y \in Y$, разложения в прямую сумму

$$
\mathbb{R}^{m}=E_{y}^{1} \oplus \cdots \oplus E_{y}^{r}, \quad \mathbb{R}^{m}=\widetilde{E}_{y}^{1} \oplus \cdots \oplus \widetilde{E}_{y}^{r},
$$

которые зависят измеримым образом от $y \in Y$ и для которых верно следуюmee. 
1. Для $\mu$-почти всех $y \in Y, n \in \mathbb{Z}$ и любого $i=1, \ldots, r$ верно

$$
\mathbb{A}(n, y) E_{y}^{i}=E_{T^{n} y}^{i}, \quad \mathbb{A}^{t}(n, y) \widetilde{E}_{y}^{i}=\widetilde{E}_{T^{-n} y}^{i} .
$$

2. Для любого $v \in E_{y}^{i}, v \neq 0$, имеет место соотношение

$$
\lim _{|n| \rightarrow \infty} \frac{\log |\mathbb{A}(n, y) v|}{n}=\theta_{i}
$$

u эта сходимость равномерна на сфере $\left\{v \in E_{y}^{i}:|v|=1\right\}$.

3. Для любого $v \in \widetilde{E}_{y}^{i}, v \neq 0$, имеет место соотношение

$$
\lim _{|n| \rightarrow \infty} \frac{\log \left|\mathbb{A}^{t}(n, y) v\right|}{n}=\theta_{i}
$$

u эта сходимость равномерна на сфере $\left\{v \in \widetilde{E}_{y}^{i}:|v|=1\right\}$.

4. Для любого $\varepsilon>0$ существуют положительные измеримые функции

$$
C_{\varepsilon, 1}^{(i)}, C_{\varepsilon, 2}^{(i)}, \widetilde{C}_{\varepsilon, 1}^{(i)}, \widetilde{C}_{\varepsilon, 2}^{(i)}: Y \rightarrow \mathbb{R}_{>0}
$$

такие, что для $\mu$-почти всех $у \in Y$ неравенства

$$
\begin{aligned}
& C_{\varepsilon, 1}^{(i)}(y) e^{\theta_{i} n-\varepsilon(n+|k|)} \leqslant\left\|\left.\mathbb{A}\left(n, T^{k} y\right)\right|_{E_{T^{k} y}^{i}}\right\| \leqslant C_{\varepsilon, 2}^{(i)}(y) e^{\theta_{i} n+\varepsilon(n+|k|)}, \\
& \widetilde{C}_{\varepsilon, 1}^{(i)}(y) e^{\theta_{i} n-\varepsilon(n+|k|)} \leqslant\left\|\left.\mathbb{A}^{t}\left(n, T^{k} y\right)\right|_{\widetilde{E}_{T^{k} y}^{i}}\right\| \leqslant \widetilde{C}_{\varepsilon, 2}^{(i)}(y) e^{\theta_{i} n+\varepsilon(n+|k|)}
\end{aligned}
$$

имеют место для всех $k \in \mathbb{Z}, n \in \mathbb{N}$

5. $\operatorname{dim} E_{y}^{i}=\operatorname{dim} \widetilde{E}_{y}^{i}, i=1, \ldots, r, u$ для любых векторов $v \in E_{y}^{i}, \widetilde{v} \in \widetilde{E}_{y}^{j}$, где $i \neq j$, выполнено равенство

$$
\langle v, \widetilde{v}\rangle=0
$$

Эта теорема немедленно следует из теоремы Оселедца-Песина о редукции (см. теорему 3.5.5 на с. 77 в [5]).

\section{А.2. Лемма Вианы о простоте старшего показателя Ляпунова.}

Лемма А.2 (М. Виана). Пусть $(Y, \mathscr{B}, \mu)$ - измеримое пространство, $T$ : $Y \rightarrow Y$ - обратимое эргодичное сохраняющее меру $\mu$ преобразование. Пусть $m \in \mathbb{N} u \mathbb{A}: Y \rightarrow \operatorname{GL}(m, \mathbb{R})$ - измеримое отображение, для которого функиии

$$
\log (1+\|A(y)\|) \quad u \quad \log \left(1+\left\|A^{-1}(y)\right\|\right)
$$

принадлежат пространству $L_{1}(Y, \mu)$.

Предположим, что для $\mu$-почти всех $y \in Y$ все элементы матрицы $\mathbb{A}$ положительны и, более того, существует такая константа $C>0$, что для 
$\mu$-почти всех $y \in Y$ и любых $i, j, k \in\{1, \ldots, m\}$ выполнены неравенства

$$
C^{-1}<\frac{\mathbb{A}_{i j}(y)}{\mathbb{A}_{k j}(y)}<C .
$$

Тогда старший показатель Ляпунова кочикла $\mathbb{A}$ является положительным и простьм.

Это утверждение является переформулировкой леммы 5.7 в работе М. Вианы [47]. Отметим, что наш коцикл $\mathbb{A}$ является обратным к коциклу, рассматриваемому М. Вианой. Из леммы А.2 немедленно получаем такое следствие.

СлеДСтвиЕ А.3. 1. Пусть $\mu-\sigma$-инвариантная эргодическая вероятностная мера на пространстве $\Omega$ марковских компактов. Если для $\mu$ выполнено условие 1.2, то старший показатель Ляпунова ренормализационного коиикла $\mathbb{A}$ относительно меры $\mu$ является положительным и простым.

2. Пусть $\mathrm{P}$ - эргодическая вероятностная $g_{s}$-инвариантная мера на пространстве $\bar{\Omega}$ марковских компактов с мерой. Если $\mathrm{P} \in \mathscr{P}^{+}$, то старший показатель Ляпунова ренормализационного коцикла $\overline{\mathbb{A}}$ относительно меры $\mathrm{P}$ является положительным и простым.

\section{Приложение В. Соответствие между символическими и геометрическими конструкциями}

\begin{tabular}{|l|l|}
\hline Символические объекты & Геометрические объекты \\
\hline Марковский компакт $X$ & Абелев дифференциал X \\
\hline $\begin{array}{l}\text { Асимптотическое слоение } \mathscr{F}^{+}, \\
\text {соответствующее будущему }\end{array}$ & Вертикальное слоение $\mathscr{F}^{+}$ \\
\hline $\begin{array}{l}\text { Асимптотическое слоение } \mathscr{F}^{-}, \\
\text {соответствующее прошлому }\end{array}$ & Горизонтальное слоение $\mathscr{F}^{-}$ \\
\hline $\begin{array}{l}\text { Пространства } \mathfrak{B}^{+} \text {и } \mathfrak{B}^{-} \\
\text {конечно аддитивных гёльдеровских мер }\end{array}$ & $\begin{array}{l}\text { Пространства } \mathfrak{B}^{+} \text {и } \mathfrak{B}^{-} \\
\text {гёльдеровских коциклов }\end{array}$ \\
\hline $\begin{array}{l}\text { Конечно аддитивные меры } m_{\Phi^{-}}, \\
\Phi^{-} \in \mathfrak{B}^{-}\end{array}$ & $\begin{array}{l}\text { Инвариантные распределения } \\
\text { Форни для вертикального }\end{array}$ \\
\hline Преобразования Вершика & потока \\
\hline Символические потоки $h_{t}^{+}$ & Перекладывания отрезков \\
\hline Матрицы смежности $A\left(\Gamma_{n}\right)$ & $\begin{array}{l}\text { Потоки } h_{t}^{+} \\
\text {на плоских поверхностях }\end{array}$ \\
\hline $\begin{array}{l}\text { Пространство } \bar{\Omega} \text { марковских компактов } \\
\text { с мерой }\end{array}$ & $\begin{array}{l}\text { Пространство } \mathscr{H} \text { модулей } \\
\text { абелевых дифференциалов }\end{array}$ \\
\hline Ренормализационный поток $g_{s}$ & Поток Тейхмюллера $\mathbf{g}_{s}$ \\
\hline Ренормализационный коцикл $\overline{\mathbb{A}}$ & Коцикл Концевича-Зорича $\mathbf{A}_{\mathrm{K} Z}$ \\
\hline $\begin{array}{l}\text { Произведения мер и двойственность } \\
\text { между } \mathfrak{B}^{+}, \mathfrak{B}^{-}\end{array}$ & $\begin{array}{l}\text { Произведение } \\
\text { Колмогорова-Александера } \\
\text { и двойственность Пуанкаре } \\
\text { в когомологиях }\end{array}$ \\
\hline
\end{tabular}




\section{Список литературы}

[1] B. Adamczewski, "Symbolic discrepancy and self-similar dynamics", Ann. Inst. Fourier (Grenoble), 54:7 (2004), 2201-2234.

[2] P. Arnoux, A. M. Fisher, "Anosov families, renormalization and non-stationary subshifts", Ergodic Theory Dynam. Systems, 25:3 (2005), 661-709.

[3] A. Avila, G. Forni, "Weak mixing for interval exchange transformations and translation flows", Ann. of Math. (2), 165:2 (2007), 637-664.

[4] A. Avila, M. Viana, "Simplicity of Lyapunov spectra: proof of the Zorich-Kontsevich conjecture", Acta Math., 198:1 (2007), 1-56.

[5] L. Barreira, Ya. Pesin, Nonuniform hyperbolicity. Dynamics of systems with nonzero Lyapunov exponents, Encyclopedia Math. Appl., 115, Cambridge Univ. Press, Cambridge, 2007, xiv+513 pp.

[6] P. Billingsley, Convergence of probability measures, 2nd ed., Wiley Ser. Probab. Statist. Probab. Statist., John Wiley \& Sons, Inc., New York, 1999, x+277 pp.; pyc. пер. 1-го изд.: П. Биллингсли, Сходимость вероятностных мер, Наука, М., 1977, $351 \mathrm{c}$.

[7] V.I. Bogachev, Measure theory. I, II, Springer-Verlag, Berlin, 2007, xviii+500 pp., xiv +575 pp.

[8] F. Bonahon, "Transverse Hölder distributions for geodesic laminations", Topology, 36:1 (1997), 103-122.

[9] F. Bonahon, "Geodesic laminations with transverse Hölder distributions", Ann. Sci. École Norm. Sup. (4), 30:2 (1997), 205-240.

[10] X. Bressaud, P. Hubert, A. Maass, "Persistence of wandering intervals in self-similar affine interval exchange transformations", Ergodic Theory Dynam. Systems, 30:3 (2010), 665-686.

[11] A.I. Bufetov, "Decay of correlations for the Rauzy-Veech-Zorich induction map on the space of interval exchange transformations and the central limit theorem for the Teichmüller flow on the moduli space of Abelian differentials", J. Amer. Math. Soc., 19:3 (2006), 579-623.

[12] A.I. Bufetov, "Hölder cocycles and ergodic integrals for translation flows on flat surfaces", Electron. Res. Announc. Math. Sci., 17 (2010), 34-42.

[13] А.И. Буфетов, "Эргодические интегралы потоков на плоских поверхностях", УМH, 65:6(396) (2010), 181-182; англ. пер.: A. I. Bufetov, "Ergodic integrals of translation flows on flat surfaces", Russian Math. Surveys, 65:6 (2010), 1173-1174.

[14] A.I. Bufetov, Limit theorems for translation flows, arXiv: 1212.5574; Ann. of Math. (2) (to appear).

[15] А.И. Буфетов, Б. М. Гуревич, “Существование и единственность меры с максимальной энтропией для потока Тейхмюллера на пространстве модулей абелевых дифференциалов", Матем. сб., 202:7 (2011), 3-42; англ. пер.: А. I. Bufetov, B. M. Gurevich, "Existence and uniqueness of the measure of maximal entropy for the Teichmüller flow on the moduli space of Abelian differentials", Sb. Math., 202:7 (2011), 935-970.

[16] J.-M. Dumont, T. Kamae, S. Takahashi, "Minimal cocycles with the scaling property and substitutions", Israel J. Math., 95 (1996), 393-410.

[17] A. Eskin, M. Kontsevich, A. Zorich, Lyapunov spectrum of square-tiled cyclic covers, arXiv: 1007.5330. 
[18] A. M. Fisher, "Nonstationary mixing and the unique ergodicity of adic transformations", Stoch. Dyn., 9:3 (2009), 335-391.

[19] L. Flaminio, G. Forni, "Invariant distributions and time averages for horocycle flows", Duke Math. J., 119:3 (2003), 465-526.

[20] G. Forni, "Solutions of the cohomological equation for area-preserving flows on compact surfaces of higher genus", Ann. of Math. (2), 146:2 (1997), 295-344.

[21] G. Forni, "Deviation of ergodic averages for area-preserving flows on surfaces of higher genus", Ann. of Math. (2), 155:1 (2002), 1-103.

[22] G. Forni, Sobolev regularity of solutions of the cohomological equation, 2007, 119 pp., arXiv: 0707.0940.

[23] G. Forni, A geometric criterion for the nonuniform hyperbolicity of the Kontsevich-Zorich cocycle, 2011, 46 pp., arXiv: 1009.4655.

[24] H. Furstenberg, Stationary processes and prediction theory, Ann. of Math. Stud., 44, Princeton Univ. Press, Princeton, NJ, 1960, x+283 pp.

[25] R. Gjerde, Ør. Johansen, "Bratteli-Vershik models for Cantor minimal systems associated to interval exchange transformations", Math. Scand., 90:1 (2002), 87-100.

[26] Sh. Ito, "A construction of transversal flows for maximal Markov automorphisms", Tokyo J. Math., 1:2 (1978), 305-324.

[27] T. Kamae, "Linear expansions, strictly ergodic homogeneous cocycles and fractals", Israel J. Math., 106:1 (1998), 313-337.

[28] А.Б. Каток, "Инвариантные меры потоков на ориентируемых поверхностях", Докл. АН СССР, 211 (1973), 775-778; англ. пер.: А. B. Katok, "Invariant measures of flows on orientable surfaces", Soviet Math. Dokl., 14 (1973), 1104-1108.

[29] M. Kontsevich, "Lyapunov exponents and Hodge theory", The mathematical beauty of physics (Saclay, 1996), Adv. Ser. Math. Phys., 24, World Sci. Publ., River Edge, NJ, 1997, 318-332.

[30] M. Kontsevich, A. Zorich, "Connected components of the moduli spaces of Abelian differentials with prescribed singularities", Invent. Math., 153:3 (2003), 631-678.

[31] S. Marmi, P. Moussa, J.-C. Yoccoz, "The cohomological equation for Roth-type interval exchange maps", J. Amer. Math. Soc., 18:4 (2005), 823-872 (electronic).

[32] S. Marmi, P. Moussa, J.-C. Yoccoz, Affine interval exchange maps with a wandering interval, arXiv: 0805.4737.

[33] H. Masur, "Interval exchange transformations and measured foliations", Ann. of Math. (2), 115:1 (1982), 169-200.

[34] В. И. Оселедец, "Мультипликативная эргодическая теорема. Характеристические показатели Ляпунова динамических систем", Тр. ММО, 19, Изд-во Моск. ун-та, M., 1968, 179-210; англ. пер.: V. I. Oseledets, "A multiplicative ergodic theorem. Lyapunov characteristic numbers for dynamical systems", Trans. Moscow Math. Soc., 19 (1968), 197-231.

[35] Ya. G. Sinai, C. Ulcigrai, "Weak mixing in interval exchange transformations of periodic type", Lett. Math. Phys., 74:2 (2005), 111-133.

[36] B. Solomyak, "On the spectral theory of adic transformations", Representation theory and dynamical systems, Adv. Soviet Math., 9, Amer. Math. Soc., Providence, RI, 1992, 217-230.

[37] W. A. Veech, "Gauss measures for transformations on the space of interval exchange maps", Ann. of Math. (2), 115:1 (1982), 201-242. 
[38] W.A. Veech, "The metric theory of interval exchange transformations. I. Generic spectral properties", Amer. J. Math., 106:6 (1984), 1331-1359.

[39] W.A. Veech, "The metric theory of interval exchange transformations. II. Approximation by primitive interval exchanges", Amer. J. Math., 106:6 (1984), 1361-1387.

[40] W.A. Veech, "The metric theory of interval exchange transformations. III. The Sah-Arnoux-Fathi invariant", Amer. J. Math., 106:6 (1984), 1389-1422.

[41] W. A. Veech, "The Teichmüller geodesic flow", Ann. of Math. (2), 124:3 (1986), 441-530.

[42] W.A. Veech, "Moduli spaces of quadratic differentials", J. Analyse Math., 55:1 (1990), 117-171.

[43] W. A. Veech, "Decoding Rauzy induction: Bufetov's question", Mosc. Math. J., 10:3 (2010), 647-657.

[44] А. М. Вершик, "Теорема о марковской периодической аппроксимации в эргодической теории", Краевые задачи математической физики и смежные вопросы теории функиий. 14, Зап. науч. сем. ЛОМИ, 115, Изд-во "Наука", Ленинград. отд., Л., 1982, 72-82; англ. пер.: А. М. Vershik, "A theorem on the Markov periodic approximation in ergodic theory", J. Soviet Math., 28:5 (1985), 667-674.

[45] A. M. Vershik, "The adic realizations of the ergodic actions with the homeomorphisms of the Markov compact and the ordered Bratteli diagrams", Теория представлений, динамические системы, комбинаторные и алгоритмические методы. I, Зап. науч. сем. ПОМИ, 223, ПОМИ, СПб., 1995, 120-126; J. Math. Sci. (New York), 87:6 (1997), 4054-4058.

[46] A. M. Vershik, A. N. Livshits, "Adic models of ergodic transformations, spectral theory, substitutions, and related topics", Representation theory and dynamical systems, Adv. Soviet Math., 9, Amer. Math. Soc., Providence, RI, 1992, 185-204.

[47] M. Viana, Lyapunov exponents of Teichmüller flows, Preprint IMPA, 2007, 64 pp., http://www.preprint.impa.br/FullText/Viana__Fri_Dec_29_10_54_53_BRDT_2006/lt.pdf.

[48] A. Zorich, "Deviation for interval exchange transformations", Ergodic Theory Dynam. Systems, 17:6 (1997), 1477-1499.

[49] A. Zorich, "How do the leaves of a closed 1-form wind around a surface?", Pseudoperiodic topology, Amer. Math. Soc. Transl. Ser. 2, 197, Amer. Math. Soc., Providence, RI, 1999, 135-178.

\section{А. И. Буфетов (А. I. Bufetov)}

Математический институт им. В. А. Стеклова РАН;

Институт проблем передачи информации

им. А. А. Харкевича РАН;

Национальный исследовательский университет

"Высшая школа экономики";

Laboratoire d'Analyse, Topologie, Probabilités,

Aix-Marseille Université, CNRS

E-mail: bufetov@mi.ras.ru
Поступила в редакцию

20.02.2012 